\title{
FITTING THE LINEAR-QUADRATIC MODEL TO DETAILED DATA SETS FOR DIFFERENT DOSE RANGES
}

by

Lourdes Maria Garcia-Fernández

B.Sc. in Nuclear Physics (1992)

\author{
A thesis submitted to the \\ Faculty of Graduate Studies and Research \\ in partial fulfillment of the requirements \\ for the degree of \\ Master of Science
}

Ottawa-Carleton Institute for Physics

Department of Physics, Carleton University

Ottawa, Ontario, Canada

July, 2005

(c) Copyright 2005, Lourdes M. Garcia-Fernández 


$\begin{array}{ll}\begin{array}{l}\text { Library and } \\ \text { Archives Canada }\end{array} & \begin{array}{l}\text { Bibliothèque et } \\ \text { Archives Canada }\end{array} \\ \begin{array}{l}\text { Published Heritage } \\ \text { Branch }\end{array} & \begin{array}{l}\text { Direction du } \\ \text { Patrimoine de l'édition }\end{array} \\ \begin{array}{l}\text { 395 Wellington Street } \\ \text { Ottawa ON K1A ON4 }\end{array} & \begin{array}{l}\text { 395, rue Wellington } \\ \text { Ottawa ON K1A ON4 } \\ \text { Canada }\end{array}\end{array}$

Your file Votre référence

ISBN: 0-494-10137-7

Our file Notre référence

ISBN: 0-494-10137-7

NOTICE:

The author has granted a nonexclusive license allowing Library and Archives Canada to reproduce, publish, archive, preserve, conserve, communicate to the public by telecommunication or on the Internet, loan, distribute and sell theses worldwide, for commercial or noncommercial purposes, in microform, paper, electronic and/or any other formats.

The author retains copyright ownership and moral rights in this thesis. Neither the thesis nor substantial extracts from it may be printed or otherwise reproduced without the author's permission.
AVIS:

L'auteur a accordé une licence non exclusive permettant à la Bibliothèque et Archives Canada de reproduire, publier, archiver, sauvegarder, conserver, transmettre au public par télécommunication ou par l'Internet, prêter, distribuer et vendre des thèses partout dans le monde, à des fins commerciales ou autres, sur support microforme, papier, électronique et/ou autres formats.

L'auteur conserve la propriété du droit d'auteur et des droits moraux qui protège cette thèse. $\mathrm{Ni}$ la thèse ni des extraits substantiels de celle-ci ne doivent être imprimés ou autrement reproduits sans son autorisation.
In compliance with the Canadian

Privacy Act some supporting forms may have been removed from this thesis.

While these forms may be included in the document page count, their removal does not represent any loss of content from the thesis.
Conformément à la loi canadienne sur la protection de la vie privée, quelques formulaires secondaires ont été enlevés de cette thèse.

Bien que ces formulaires aient inclus dans la pagination, il n'y aura aucun contenu manquant.

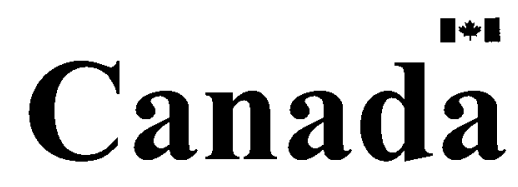




\section{Abstract}

Survival curve behaviour and degree of correspondence between the Linear Quadratic model (LQ) and experimental data in an extensive dose range for high dose rates were analyzed. Detailed clonogenic assays with irradiation given in $0.5 \mathrm{~Gy}$ increments and a total dose range varying from 10.5 to 16 Gy were performed. The cell lines investigated were: CHOAA8 (Chinese hamster fibroblast cells), U373MG (human glioblastoma cells), CP3 and DU145 (human prostate carcinoma cell lines). The analyses were based on $x^{2}$-statistics and Monte Carlo simulation of the experiments.

A decline of LQ fit quality at very low doses ( $<2 \mathrm{~Gy})$ is observed. This result can be explained by the hypersensitive effect observed in CHOAA8, U373MG and DU145 data and an adaptive type response in the CP3 cell line. A clear improvement of the fit is discerned by removing the low dose data points. The fit worsening at high doses also shows that LQ cannot explain this region. This shows that the LQ model fits better the middle dose region of the survival curve. 
The analysis conducted in our study reveals a dose dependency of the LQ fit in different cell lines. The $\alpha / \beta$ ratios resulting from the LQ fit with and without low dose data points show differences of $42,40,23$ and $24 \%$ for CHOAA8, CP3, U373MG and DU145 respectively. 


\section{Acknowledgements}

This thesis would not appear in its present form without the kind assistance and support of many individuals and organizations:

I am indebted to my supervisors, Dr. G. Peter Raaphorst and Dr. David Wilkins, for the guidance and support they have provided throughout the course of this work. Their patience, despite my many, many questions, is greatly appreciated.

I would also like to express my gratitude to all people in the Medical Physics Department at the Ottawa Regional Cancer Centre for making my stay enjoyable and memorable.

I would especially like to thank Julie Leblanc for her assistance and help with all the long experiments involved in my thesis.

I would like to thank the National Cancer Institute of Canada for supporting this research through a NCIC grant.

I would like to thank my professors at Carleton University, Gerald Oakham and Paul Johns for sharing their wide knowledge and for the constant 
encouragement. I would especially like to thank Alain Bellerive and Dave Rogers for their valuable feedback and advice.

Huge thanks to my colleagues and friends for helping and assisting me during the period of my master's and the preparation of this thesis. Their honest yet considerate criticisms of this work have helped much in improving its quality. Special thanks to Mihai Gherase, Sorina Truica and Mike Donkers.

Thanks from my heart to my family, for their love, support and encouragement always provide to me.

Finally the greatest thanks to my daughter Daniela for understanding when instead of playing or reading a good night story I was doing experiments or working on this thesis.

vi 


\section{Contents}

Abstract iii

Acknowledgements $\quad$ V

$\begin{array}{ll}\text { 1. Introduction } & 1\end{array}$

1.1 Response of Cells to radiation..................................... 1

1.2 Survival Curve .......................................................... 2

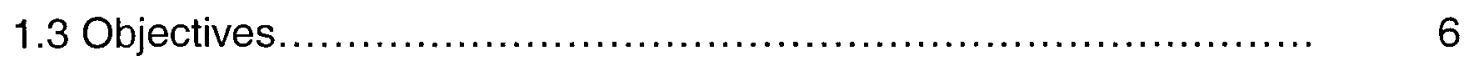

$\begin{array}{ll}\text { 2. Theory } & 8\end{array}$

2.1 Linear Quadratic Model................................................ 9

2.1.1 A molecular Theory of Cell Survival based on LQ Model........ 9

2.1.2 The Lea-Catcheside Dose Protraction Factor................... $\quad 15$

2.1.3 The $\alpha / \beta$ Ratio. Definition and Importance...................... 16

2.1.4 Shortcoming of LQ Model.................................... 18

$\begin{array}{ll}\text { 3. Materials and Methods } & 21\end{array}$

3.1 Clonogenic Assays.................................................. 21

3.1.1 Cell lines and Culture........................................ 21

vii 
3.1.2 Irradiation Procedure ........................................... 22

3.1.3 Clonogenic Survival........................................... 22

3.1.4 Multiplicity Correction....................................... $\quad 25$

3.2 Data Analysis.................................................... 27

3.2.1 Least Squares Fitting Method. Goodness of Fit................ $\quad 29$

3.2.2 Parameter Estimation and Confidence Regions in LSM........ $\quad 34$

3.2.3 Introduction to Monte Carlo Method ............................. $\quad 37$

3.2.4 Monte Carlo Method for Simulation. Modeling.................... 38

4. Results and Discussion $\quad 42$

4.1 Multiplicity Correction................................................ 43

4.2 Experimental Survival Curves.......................................... 44

4.2.1 Hamster Fibroblast Survival Curve. CHOAA8 Cell line.......... $\quad 47$

4.2.2 Human Glioma Cell Survival Curve. U373MG Cell line......... 48

4.2.3 Human Prostate Cancer Cell Survival Curve.

CP3 Cell line................................................. 49

4.2.4 Human Prostate Cancer Cell Survival Curve.

DU145 Cell line ............................................ 50

4.2.5 Discussion ................................................. 52

4.2.5.1 Survival Curves Uncertainties......................... 52

4.2.5.2 Survival Curves. General Trend....................... $\quad 55$

4.3 Data Analysis Results............................................... 57

4.3.1 Results of Data Analysis for CHOAA8 Cell line................. 58

4.3.1.1 Evaluating Goodness of Fit for CHOAA8 Cell line..... 58

4.3.1.2 Monte Carlo Simulation for CHOAA8 Cell line......... 62

viii 
4.3.1.3 Estimated Parameter for CHOAA8 Cell line........... 66

4.3.1.4 Discussion...................................... 70

4.3.2 Results of Data Analysis for CP3 Cell line.................. 79

4.3.2.1 Evaluating Goodness of Fit for CP3 Cell line.......... 79

4.3.2.2 Monte Carlo Simulation for CP3 Cell line............. 82

4.3.2.3 Estimated Parameter for CP3 Cell line................ 86

4.3.2.4 Discussion................................... 90

4.3.3 Results of Data Analysis for U373MG Cell line.............. 93

4.3.3.1 Evaluating Goodness of Fit for U373MG Cell line..... 93

4.3.3.2 Monte Carlo Simulation for U373MG Cell line........ 96

4.3.3.3 Estimated Parameter for U373mg Cell line............ 100

4.3.3.4 Discussion....................................... 104

4.3.4 Results of Data Analysis for DU145 Cell line............... 107

4.3.4.1 Evaluating Goodness of Fit for DU145 Cell line....... 107

4.3.4.2 Monte Carlo Simulation for DU145 Cell line........... 110

4.3.4.3 Estimated Parameter for DU145 Cell line.............. 114

4.3.4.4 Discussion.................................... 118

4.4 Impact of Initial Linear Region in $\alpha / \beta$ Ratio....................... 121

5. Conclusions and Future Directions $\quad 127$

5.1 Conclusions................................................... 127

5.2 Future Directions................................................. 129

$\begin{array}{ll}\text { References } & 130\end{array}$

ix 


\section{List of Tables}

4.1 Multiplicity factor for the studied cell lines..........................

4.2 Comparison of uncertainties obtained from different clonogenic survival assays. Our experiment and other similar data are included....

4.3 Surviving fractions and standard error of the mean from clonogenic survival for several cell lines at doses of 2 Gy and $10 \mathrm{~Gy}$

4.4 $X^{2}$-Test for CHOAA8 cell line at several dose ranges. Testing the LQ hypothesis for a significance level of $5 \%$. In the last column the $P$-value is also shown.

4.5 Expectations for the theoretical and MC $x^{2}$-distributions at several dose ranges. The difference is also displayed in the last column

4.6 The parameters $\alpha$ and $\beta$ from the $L Q$ model least square fit to the CHOAA8 survival data, and their corresponding standard deviation $\sigma_{\alpha}$ and $\sigma_{\beta}$. The $x^{2} /$ df statistics from the fit and the correlation coefficient between $\alpha$ and $\beta$ are also given.

4.7 Chi-Square divided by degrees of freedom at different dose ranges and excluding 4 Gy data point in the first two columns and excluding 13.5 Gy in the last two columns for CHOAA8. The points are excluded due to the fact that they may be outliers.

4.8 $\quad x^{2}$-Test for CP3 cell line at several dose ranges. Testing the LQ hypothesis for a significance level of $5 \%$. In the last column the $P$-value is also shown. 
4.9 Expectations for the theoretical and $M C x^{2}$-distributions at several dose ranges for CP3 cell line. The difference is also displayed in the last column

4.10 The parameters $\alpha$ and $\beta$ from LQ model least square fit to the CP3 survival data, and their corresponding standard deviation $\sigma_{\alpha}$ and $\sigma_{\beta}$. The $x^{2} /$ df statistics from the fit and the correlation coefficient between $\alpha$ and $\beta$ are also given.

4.11 $\mathrm{X}^{2}$-Test for U373MG cell line at several dose ranges. Testing the LQ hypothesis for a significance level of $5 \%$. In the last column the $\mathrm{P}$-value is also shown

4.12 Expectations for the theoretical and $M C x^{2}$-distributions at several dose ranges for U373MG cell line. The difference is also displayed in the last column

4.13 The parameters $\alpha$ and $\beta$ from LQ model least square fit to the U373MG survival data, and their corresponding standard deviation $\sigma_{\alpha}$ and $\sigma_{\beta}$. The $\chi^{2} /$ df statistics from the fit and the correlation coefficient between $\alpha$ and $\beta$ are also given.

4.14 $x^{2}$-Test for DU145 cell line at several dose ranges. Testing the LQ hypothesis for a significance level of $5 \%$. In the last column the $P$-value is also shown

4.15 Expectations for the theoretical and $M C x^{2}$-distributions at several dose ranges. The difference is also displayed in the last column.................................................

4.16 The parameters $\alpha$ and $\beta$ from LQ model least square fit to the DU145 survival data, and their corresponding standard deviation $\sigma_{\alpha}$ and $\sigma_{\beta}$. The $x^{2} /$ df statistics from the fit and the correlation coefficient between $\alpha$ and $\beta$ are also given 


\section{List of Figures}

1.1 Survival Curve for HeLa cell line [T.T. Puck and P.I. Marcus 1956 (Ref. 4)]

1.2 Clonogenic Survival Curves for DU145 and CP3 cell lines. [O. Algan et al. 1996 (Ref. 25)]

1.3 Clonogenic survival Curve for $\mathrm{CHO}$ cell line [D. Bartkoviak et al. 2001 (Ref. 26)]

2.1 Schematic representation of the dsb by: (a) one-track event, and (b) two-track events

2.2 Illustration of the two components that make up the linear quadratic model. The linear component is $(-\log S=a D)$; the

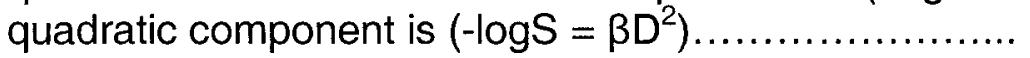

3.1 Schematic representation of a plated cell before and after cellular division with a multiplicity factor $\bar{N}=2$.

3.2 Dose ranges definition. Fixing the lower bound of the range at 0 Gy, the upper bound changes every 0.5 Gy, keeping at least 4 Gy as the minimal range. Next, the lower bound will move to the right also every $0.5 \mathrm{~Gy}$, each time repeating the same procedure described above.

4.1 Survival Curve for U373MG cell line from clonogenic

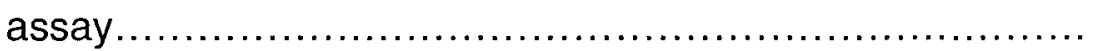

4.2 Stained colonies: (a) Control for CHOAA8, (b) High dose for CHOAA8, (c) Control for CP3, (d) High dose for CP3... 
4.3 Survival Curve for CHOAA8 cell line from clonogenic

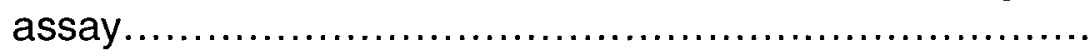

4.4 Survival Curve for CP3 cell line from clonogenic assay...... 50

4.5 Survival Curve for DU145 cell line from clonogenic assay.. $\quad 51$

4.6 Chi-Square divided by degrees of freedom at different dose ranges for CHOAA8 cell line. Initial dose of the range is fixed at $0,2.5$ and $5.5 \mathrm{~Gy}$. Final dose of the range varies along the $\mathrm{X}$-axis.

4.7 Chi-Square divided by degrees of freedom at different dose ranges for $\mathrm{CHOAA8}$. Final dose of the range is fixed at $13 \mathrm{~Gy}$. Initial dose of the range varies along $X$-axis ......

4.8 Plots of Chi-Square distribution from Monte Carlo experiment for CHOAA8 in dark bars and the theoretical expected Chi-Square distribution in solid lines. The graphs show the distributions for different dose ranges (see the upper right corner of each graph)

4.9 P-value distribution from Monte Carlo experiment at different dose ranges for CHOAA8. One can see an evident difference among the different dose ranges although the complete flat distribution for $P$-value is not achieved

4.10 Alpha parameters for the LQ model obtained from LSM. Initial dose of the range is fixed at $0,2.5$ and $5.5 \mathrm{~Gy} . . . . .$.

4.11 Beta parameters for the LQ model obtained from LSM. Initial dose of the range is fixed at 0, 2.5 and 5.5 Gy........

4.12 LQ fit at different dose ranges for CHOAA8 cell line. The $\mathrm{LQ}$ models are extrapolated to the total dose range.

4.13 Chi-Square divided by degrees of freedom at different dose ranges and excluding 4 Gy data point for CHOAA8. Final dose of the range is fixed at 13 Gy. Initial dose of the range varies along $X$-axis 
4.14 Residuals from LQ fit for CHOAA8. The plots show the total range investigated (upper-left corner) and a more detailed behavior of the same residuals for smaller dose scales: (0-6) Gy in upper-right corner, (6-10) Gy in lowerleft corner, (10-16) Gy in lower-right corner...................

4.15 Chi-Square divided by degrees of freedom at different dose ranges for CP3 cell line. Initial dose of the range is fixed at $0,2.5$ and $5.5 \mathrm{~Gy}$. Final dose of the range varies along $X$-axis

4.16 Chi-Square divided by degrees of freedom at different dose ranges for CP3. Final dose of the range is fixed 11.5 Gy. Initial dose of the range varies along $X$-axis

4.17 Plots of Chi-Square distribution from Monte Carlo experiment for CP3 in dark bars and the theoretical expected Chi-Square distribution in solid lines. The graphs show the distributions for different dose ranges (see the upper right corner of each graph).

4.18 P-value distribution from Monte Carlo experiment at different dose ranges for $\mathrm{CP} 3$. One can see an evident difference among the different dose ranges although the complete flat distribution for $\mathrm{P}$-value is not achieved.........

4.19 Alpha parameters for the LQ model obtained from LSM. Initial dose of the range is fixed at 0, 2.5 and 5.5 Gy. CP3 cell line.

4.20 Beta parameters for the LQ model obtained from LSM. Initial dose of the range is fixed at 0, 2.5 and 5.5 Gy. CP3 cell line

4.21 LQ fit at different dose ranges for CHOAA8 cell line. The LQ models are extrapolated to the total dose range.

4.22 Residuals from LQ fit for CP3. The plots show the total range investigated (upper-left corner) and a more detailed behaviour of the same residuals for smaller dose scales: (0-5) Gy in upper-right corner, (5-11) Gy in lower-left corner, (10-14) Gy in lower-right corner. 
4.23 Chi-Square divided by degrees of freedom at different dose ranges for U373MG cell line. Initial dose of the range is fixed at $0,2.5$ and 4 Gy. Final dose of the range varies along $\mathrm{X}$-axis

4.24 Chi-Square divided by degrees of freedom at different dose ranges for U373MG. Final dose of the range is fixed 9.5 Gy. Initial dose of the range varies along $X$-axis

4.25 Plots of Chi-Square distribution from Monte Carlo experiment for U373MG in dark bars and the theoretical expected Chi-Square distribution in solid lines. The graphs show the distributions for different dose ranges (see the upper right corner of each graph)

4.26 P-value distribution from Monte Carlo experiment at different dose ranges for U373MG. One can see an evident difference among the different dose ranges although the complete flat distribution for P-value is not achieved

4.27 Alpha parameters for the LQ model obtained from LSM. Initial dose of the range is fixed at $0,2.5$ and $4 \mathrm{~Gy}$. U373MG cell line

4.28 Beta parameters for the LQ model obtained from LSM. Initial dose of the range is fixed at $0,2.5$ and 4 Gy. U373MG cell line

4.29 LQ fit at different dose ranges for U373MG cell line. The LQ models are extrapolated to the total dose range

4.30 Residuals from LQ fit for U373MG. The plots show the total range investigated (upper-left corner) and a more detailed behaviour of the same residuals for smaller dose scales: (0-4) Gy in upper-right corner, (4-10) Gy in lowerleft corner, (7-9.5) Gy in lower-right corner

4.31 Chi-Square divided by degrees of freedom at different dose ranges for DU145 cell line. Initial dose of the range is fixed at 0, 2, 4 and 6 Gy. Final dose of the range varies along $\mathrm{X}$-axis 
4.32 Chi-Square divided by degrees of freedom at different dose ranges for DU145. Final dose of the range is fixed at 7.5 and 13 Gy. Initial dose of the range varies along $X$ axis.

4.33 Plots of Chi-Square distribution from Monte Carlo experiment for DU145 in dark bars and the theoretical expected Chi-Square distribution in solid lines. The graphs show the distributions for different dose ranges (see the upper right corner of each graph).............................

4.34 P-value distribution from Monte Carlo experiment at different dose ranges for DU145. One can see an evident difference among the different dose ranges although the complete flat distribution for P-value is not achieved

4.35 Alpha parameters for the LQ model obtained from LSM. Initial dose of the range is fixed at 0 and 2.5 Gy. DU145 cell line

4.36 Beta parameters for the LQ model obtained from LSM. Initial dose of the range is fixed at 0 and 2.5 Gy. DU145 cell line

4.37 LQ fit at different dose ranges for DU145 cell line. The LQ models are extrapolated to the total dose range

4.38 Residuals from LQ fit for DU145. The plots show the total range investigated (upper-left corner) and a more detailed behaviour of the same residuals for smaller dose scales: (0-5) Gy in upper-right corner, (2-8) Gy in lower-left corner, (8-14) Gy in lower-right

4.39 Plot of $\alpha / \beta$ ratio for CHOAA8 cell line ..................... 122

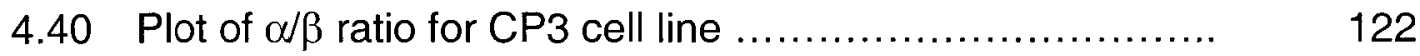

4.41 Plot of $\alpha / \beta$ ratio for U373MG cell line ..................... 123

4.42 Plot of $\alpha / \beta$ ratio for DU145 cell line ........................ 123

$4.43 \alpha / \beta$ ratio including and not including low dose data points .. 


\section{Glossary}

LQ

Linear Quadratic model.

LQL:

Linear-Quadratic-Linear model.

$\alpha$ :

Parameter in LQ model.

$\beta$ :

Parameter in LQ model.

$\mathrm{G}(\mu, \mathrm{t}):$

The Lea-Catcheside dose protraction factor.

$\alpha / \beta$ :

Ratio of the two parameters of LQ model.

DNA:

Deoxyribonucleic acid.

dsb:

Double-strand-break in DNA.

ssb :

Single-strand-break in DNA.

HRS:

Hyper radiosensitive effect.

RR:

Radio Resistant effect.

PE:

Plating Efficiency.

$\mathrm{F}$ :

Surviving Fraction.

S:

Surviving Fraction corrected by multiplicity factor.

$\bar{N}$ :

Multiplicity factor.

LET:

Linear Energy Transfer.

Dose range:

Dose range used in the fit.

xvii 
Initial dose of the range: Lower bound of the range.

Final dose of the range: Upper bound of the range.

Very low dose region: Region in the survival curve defined by the very low doses that are characterized by the presence of HRS/RR or adaptive type responses.

Low dose region:

Region in the survival curve defined by the low doses and where $L Q$ model is not fitting well.

Middle dose range: $\quad$ Region in the survival curve defined at middle doses and characterized by LQ behaviour.

High dose region: Region in the survival curve defined by the high doses and where LQ model is not fitting well.

HeLa:

Human cancer of the Cervix cell line.

CHOAA8:

Chinese Hamster Ovary cell line.

CP3:

Human Prostate Cancer cell line.

U373MG:

Human Glioma cell line.

DU145:

Human Prostate Cancer cell line.

LSM:

Least Square Method.

$\alpha_{s 1}:$

Significance level

$x^{2} / \mathrm{df}:$

Chi-Square divided by the number of degrees of freedom. 


\section{Chapter 1}

\section{INTRODUCTION}

One of the principal aims of radiation biology is to explain observed phenomena related with the therapeutic uses of radiation. A good model that represents the processes involved in the radiation interaction with cells, tissues and organs, and their radiation response would give the scientific community a better tool for the improvement of radiotherapy treatment.

\subsection{Response of Cells to Radiation}

When living tissues are exposed to ionizing radiation they undergo changes resulting in a reduction of the number of viable cells. The absorption of radiation in biological tissues leads to the immediate production of ionized and excited atoms, which could directly interact damaging critical targets. They could also break the chemical bonds of the surrounded material forming highly reactive free radicals, which are able to diffuse and react with the targets producing a chain of 
processes leading to irreversible damage. ${ }^{1}$ It is widely believed that the damage which is most significant for mutation and loss of cell viability, is damage in DNA. $^{2,3}$

The quantification of cell killing depends on the type of cell and its normal function. For cells that are not actively proliferating, cell death can be defined as loss of function. On the other hand, for cells that are actively dividing, cell death is defined as its loss of ability to divide and multiply. For cancer cells for example, one of the most important characteristics is the ability to multiply (production of viable progeny); as a consequence, the loss of ability to undergo mitosis would represent the cancer cell death. ${ }^{3}$

\subsection{Survival Curve}

In radiobiology, one of the most important concepts is cell survival. Radiation has many effects on cells; among them is the loss of colony forming ability. The proportion or fraction of cells that remains able to form colonies of daughter cells after radiation exposure is called the cell survival fraction.

From the point of view of tumour eradication and sparing of normal cells, survival can be used to describe the sensitivity of cells to radiation through the "cell survival curve"; that is a plot of surviving fraction against dose. ${ }^{1,3}$ Survival curves in vitro can just be used to guide clinical response on relative bases because single cells growing in a petri dish do not behave exactly like in the tissue. The 
cell growth rate, cycle, communication, temperature and environment and hence its quantitative dose response differ. However, the knowledge of cell radiosensitivity and the general shape of survival curve are very useful. Establishing dose-response relationship makes possible to compare dose responses in different cell lines, dose rates, milieu, and the effects of different radiosensitizers, fractionation schemes or oxygen and cycle effects among others.

Figure 1.1 shows a typical example and the first mammalian cell survival curve for HeLa cells exposed to X-rays. ${ }^{4}$ The surviving fraction is plotted on a logarithm scale vs. radiation dose. This cell line is represented by a curve that has a small initial shoulder.

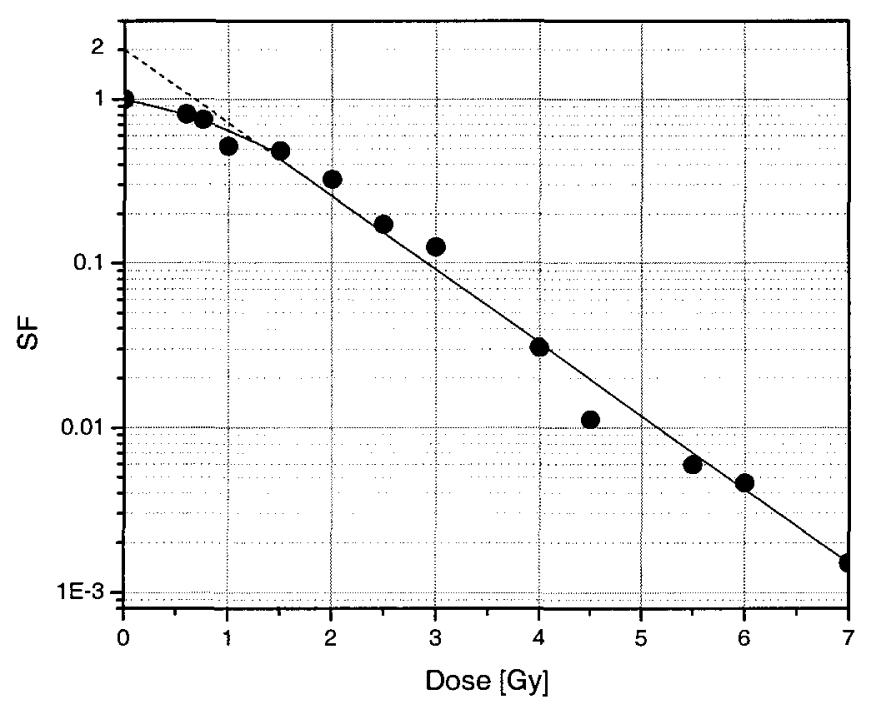

Fig.1.1 Survival Curve for HeLa cell line [T.T. Puck and P. I. Marcus 1956 (Ref. 4)] 
The general response in terms of survival curve shape could be described by the presence of an initial slope at low doses, followed by a shoulder and a final slope at the end of the survival curve. ${ }^{1,5}$ However, this shape varies depending on different factors, such as, cell line, radiation type and radiation dose rate. ${ }^{1,3}$

The shapes of the survival curve and their models on a biological basis have been the subjects of extensive investigations. Many models have successfully fitted the experimental data; however a universal theory of radiation cell killing is not yet realized. ${ }^{1,3,6-8}$

One of the most extensively used models for cell survival analysis ${ }^{5,9-12}$ and for studying radiobiological end-points ${ }^{13-15}$ is the Linear Quadratic model (LQ). It properly describes the survival curve with a very simple equation and with just two basic mechanisms of cell killing: non-repairable lesion and repairable lesion exchange. ${ }^{16}$ In addition, it can also offer a credible explanation of fractionation effects observed in radiotherapy, which illustrates its applicability in comparing and switching among different radiotherapy regimes. ${ }^{17-24}$

How the LQ formalism can describe dose-response relations produced by acute dose delivery regimen and compare different cell radiosensitivity is presented in Figs.1.2 and 1.3. The figure shows the experimental survival curves in vitro for DU145, CP3 and $\mathrm{CHO}$ cell lines. The data were published by Algan $^{25}$ and Bartkoviak, ${ }^{26}$ respectively. 


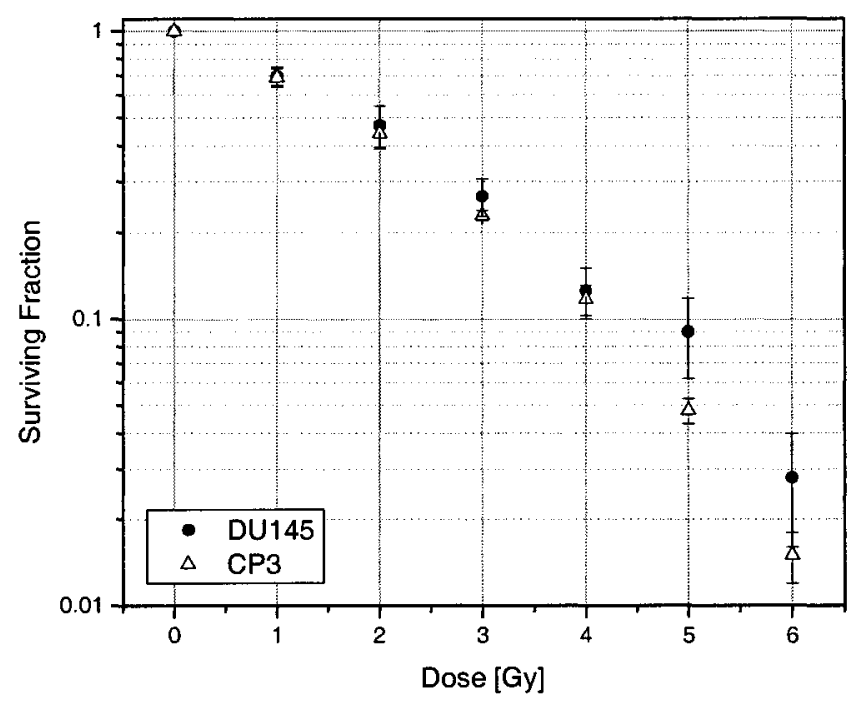

Fig.1.2 Clonogenic Survival Curves for DU145 and CP3 cell lines. [O. Algan et al. 1996 (Ref. 25)]

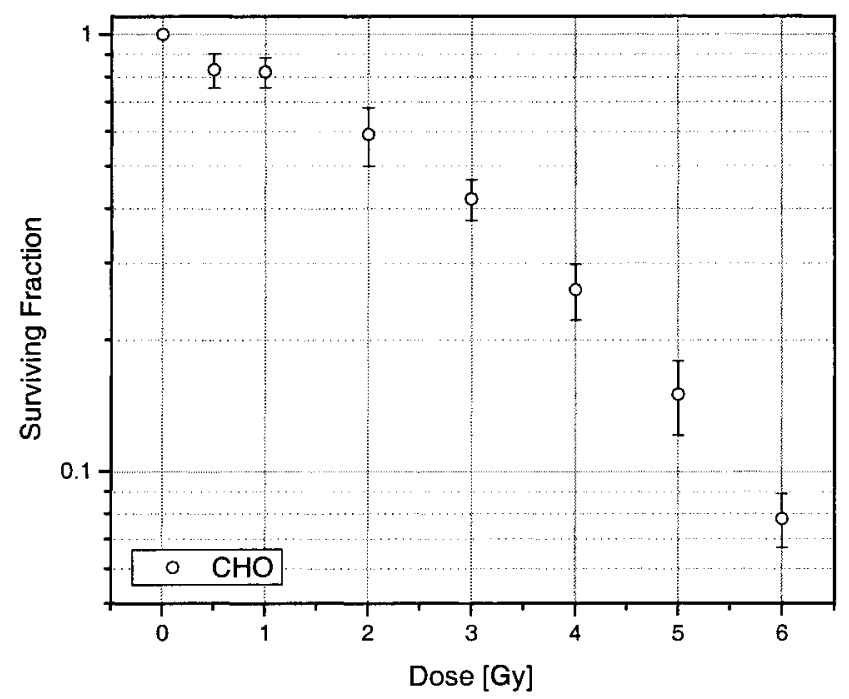

Fig.1.3 Clonogenic survival Curve for CHO cell line [D. Bartkoviak et al. 2001 (Ref. 26)] 
These curves are representative examples of the cell survival data published, characterized by a short dose range, around 6 to 10 Gy and increments of 1 or 2 Gy. More detailed information could rarely be found, and it would be mostly within the first 2 Gy dose range, thus limiting the usefulness of the data in developing and fitting cell survival models.

\subsection{Objectives}

Starting from the general survival shape, it could be expected that there is dose range dependency of the $L Q$ fit. Therefore, the aim of this research study is to analyze the survival behaviour and quantify the degree of correspondence between LQ model and experimental data in an extensive dose range for $\mathrm{X}$ radiation at dose rates comparable to those used in therapy. Since the data found in the literature were not detailed enough for a wide dose range, conventional but very detailed clonogenic assays in a broad range of dose for several cell lines are performed.

The analyses are based on goodness of fit and Monte Carlo simulations of the experiments. Under the assumption of Gaussian errors, the LQ model behaviour at different dose ranges for all the cell lines is studied. $x^{2}$-values divided by the number of degrees of freedom and observed P-values are determined. Hypothesis tests for $5 \%$ significance level are also performed. In addition, one thousand experiments are generated applying Monte Carlo technique to estimate 
the difference between the expected and the Monte Carlo simulated ' $\mathrm{X}$ ' and ' $\mathrm{P}$ value' distributions.

The influence of data dose range in fitted parameters and the consequences in a more clinical oriented $\alpha / \beta$ ratio will also be evaluated. 


\section{Chapter 2}

\section{THEORY}

The Linear Quadratic model has been widely used since it successfully describes the tendency of cell survival with a very simple equation and with just two basic mechanisms of cell killing: lethal lesion and sub-lethal lesion exchange.

The formalism, when applied to more frequently used radiotherapy doses has been shown to have significant mechanistic basis, especially for low LET radiation at not very high doses. ${ }^{27}$ Even though there are some limitations, the LQ approach is a practical tool with a successful applicability in fractionated radiotherapy ${ }^{17-24}$ giving very simple and fundamental information of radiobiological damage. The basis of its development and some limitations are presented below. 


\subsection{Linear Quadratic Model}

The model is represented by the simple equation:

$$
S=\exp \left(-\alpha D-G(\mu, t) \beta D^{2}\right)
$$

$S$ is the surviving fraction, $D$ is the radiation dose, $\alpha$ and $\beta$ are constants. $G(\mu, t)$ is the Lea-Catcheside dose protraction factor $^{28}$ between 0 and 1 , taking into account the dose rate and repair constant, $\mu$; and $t$ is the irradiation time.

The first term of the exponent is a linear component (when plotted on semilog graph) of the cell killing. It represents the amount of non-repairable damage leading irrevocably to cell death and defines the initial slope of the cell survival curve. The second one is the quadratic term, which defines its curvature. It represents the process of repairable lesion exchanges.

\subsubsection{A Molecular Theory of Cell Survival based on LQ model}

Chadwick and Leenhouts, ${ }^{16}$ and Kellerer and Rossi ${ }^{28}$ were the precursors of this theory. The biological basis of Chadwick and Lernout's model comes from the idea that the linear component would represent the spatially random production of lethal lesions that might be produced from direct induction of double-strandbreak (dsb) in the DNA molecule, which means that every dsb is a result of one 
radiation event. That kind of damage is called a lethal or non-repairable lesion. Fig.2.1.a. illustrates this idea.
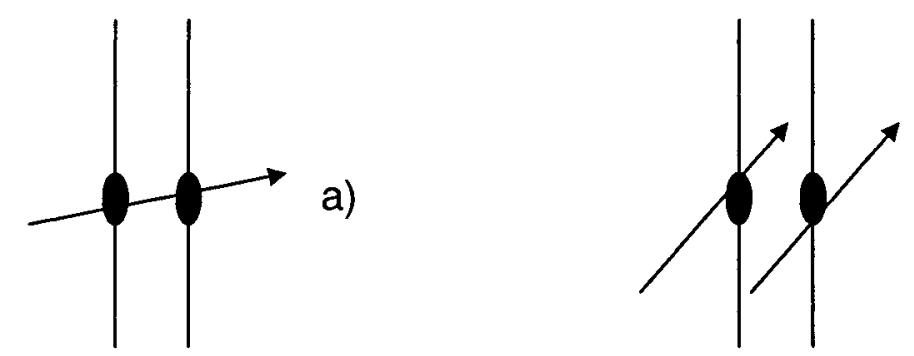

b)

Fig.2.1 Schematic representation of the dsb by: (a) one-track event, and (b) two-track events.

On the other hand, the quadratic component is a result of two single-strandbreaks (ssb) or sub-lethal lesions coming from two different radiation events, but both ssb are close enough producing a dsb, Fig.2.1.b. Since a ssb could be repaired if the cell has time before a new sub-lethal lesion is produced, this damage represents the number of repairable or sub-lethal lesion exchanges. The respective linear and quadratic components are shown in Fig.2.2. 


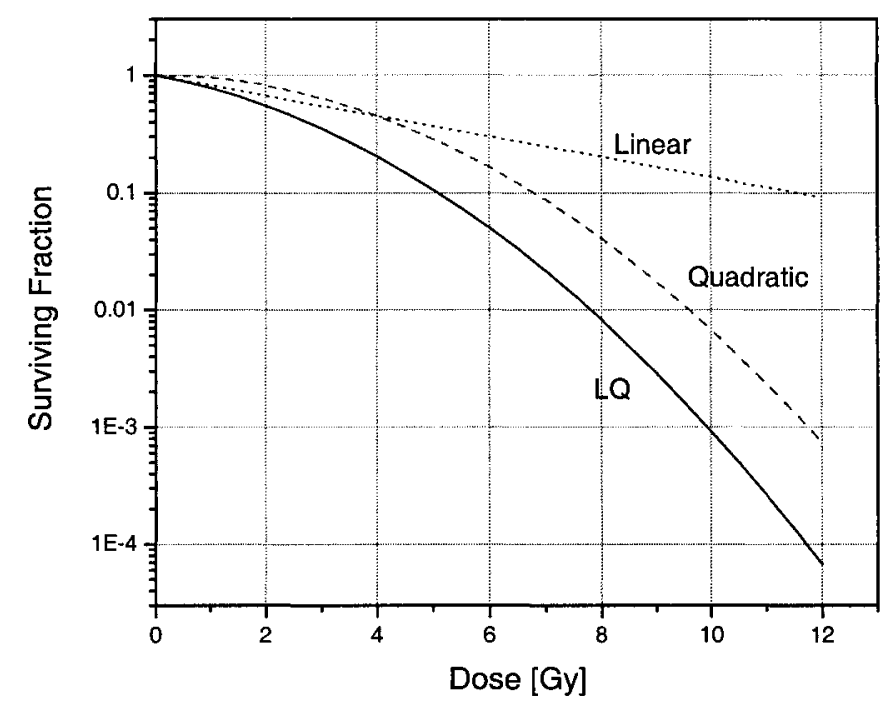

Fig.2.2 Illustration of the two components that make up the linear quadratic model. The linear component is $(-\log S=\alpha D)$; the quadratic component is $\left(-\log S=\beta D^{2}\right)$.

The assumptions behind the theory that Chadwick and Leenhout presented are summarized as:

- The critical target is the DNA (double helix)

- The critical damage leading to the cell death is a double strand break.

- Radiation produces a breakage of molecular bonds in the DNA strands.

- The broken bonds in a DNA strand can be repaired or restituted.

Starting with the assumptions above the model is developed as follow:

First, the number of DNA single strand breaks is obtained. The number of ssb per unit dose is: 


$$
\frac{d N}{d D}=-k N
$$

And the number of bonds remaining $N$ after the administered dose $\mathrm{D}$ is equal to:

$$
N=N_{1} e^{-k D}
$$

where $\mathrm{k}$ is the probability per bond and unit dose of ssb formation and $N_{1}$ is the number of bonds in the first strand. Thus, the number of broken bonds in the first strand is:

$$
N_{1}^{s s b}=N_{1}-N=N_{1}\left(1-e^{-k D}\right)
$$

Similarly for the number of broken bonds in the second strand, $N_{2}^{s s b}$, and the number of direct double strand breaks, $N_{\text {direct }}^{\text {dsb }}$, produced are:

$$
\begin{aligned}
& N_{2}^{s s b}=N_{2}-N=N_{2}\left(1-e^{-k D}\right) \\
& N_{\text {direct }}^{d s b}=N_{0}-N=N_{0}\left(1-e^{-k_{0} D}\right)
\end{aligned}
$$

where $\mathrm{k}_{0}$ is the probability per bond and per unit dose of producing a dsb. $N_{0}$ is the number of sites for potentials dsb $\left(N_{0} \leq N_{1}, N_{2}\right)$.

Considering as $\varepsilon$, the probability that two ssb are close enough to interact, the number of dsb produced by interaction of $s s b$ is: 


$$
N_{\text {inter }}^{d s b}=N_{1} N_{2} \varepsilon\left(1-e^{-k D}\right)^{2}
$$

The total number of dsb is:

$$
N_{\text {total }}^{d s b}=N_{0}\left(1-e^{-k_{0} D}\right)+N_{1} N_{2} \varepsilon\left(1-e^{-k D}\right)^{2}
$$

There is experimental evidence that lesion formation follows a Poisson distribution given by the correspondence of the number of chromosomal aberrations and the natural logarithm of cell survival. ${ }^{29}$ Therefore, assuming that lesion formation follows a Poisson distribution, the probability that no lesions are formed is given by Poisson distribution with zero number of lesions $(n=0)$ and expected number of lesions equal $\lambda$. Then, the survival can be expressed as:

$$
\begin{aligned}
& S=\frac{\lambda^{n}}{n !} e^{-\lambda} \\
& S=e^{-\lambda}
\end{aligned}
$$

Combining Eqs.2.8 and 2.10 the survival probability is given by:

$$
S=e^{-N_{0}\left(1-e^{-k_{0} D}\right)-N_{1} N_{2} \varepsilon\left(1-e^{k D}\right)^{2}}
$$

If $k_{0}$ and $k$ are assumed to be very small, we can expand in Maclaurin series the exponential term and keeping the first two terms we obtain:

$$
S=e^{-N_{0} k_{0} D-N_{1} N_{2} \varepsilon k^{2} D^{2}}=e^{-\alpha D-\beta D^{2}}
$$


where $\alpha=N_{0} k_{0}$ and $\beta=N_{1} N_{2} \varepsilon k^{2}$

For simplicity the derivation above has some omissions compared with the original obtained by Chadwick. It was developed by Carlone ${ }^{30}$ and does not take into account cell repairing mechanism while Chadwick considered the repair ability as a constant factor reducing the number of ssb.

Further research showed that ssb does not have serious biological consequences associated with cell killing since it can be easily repaired using the information from the opposite strand. On the other hand, the dsb becomes the most important type of lesion highly correlated with chromosomal aberration induction. $^{31,32}$ The dsb can also be repaired through a non-homologous or homologous recombination repair mechanism..$^{33,34}$

From the new standpoint, the LQ formalism represents the lethal lesions produced by one or two radiation events as before. However, the linear component now stands for the chromosomal aberration created by two dsb's induced by a single radiation event while the quadratic component represents the chromosomal aberration from the interaction of two repairable dsb's.

This mechanistic interpretation has been a polemic issue in the realm of radiobiology. ${ }^{27,}{ }^{35-40}$ At the beginning the LQ approach was considered an empirical relationship that successfully fits a wide range of experimental cell survival data. The significance increased as the result of connection between $\alpha / \beta$ 
ratio and 'early' and 'late' effects. The analysis of time-dose relationships in fractionation regime is consistent with the data under the assumption of linearquadratic dose-effect approach that will be presented later on.

\subsubsection{The Lea-Catcheside Dose Protraction Factor}

The Lea-Catcheside dose protraction factor, $G(\mu, t)$, is introduced to take into account cell repair capacity. The function $G$ has been derived using different methods and both are originally due to Douglas Lea. ${ }^{28,36,41,42}$

For a constant dose rate $\mathrm{R}$, the protraction factor $\mathrm{G}$ becomes the well-known expression:

$$
G(\mu, t)=\frac{2}{\mu t}\left[1-\frac{1}{\mu t}\left(1-e^{-\mu t}\right)\right]
$$

Here $\mu$ is the repair rate constant and $t$ is the irradiation time.

Applying L'Hopital's rule it can be seen that $G(\mu, t)=1$ when $\mu t \rightarrow 0$. In the case of acute delivery regimen and high dose rate, when there is no time for repairing, $G$ is considered equal to one. 


\subsubsection{The $\alpha / \beta$ Ratio. Definition and Importance}

The next important concept highly employed in radiotherapy and coming from the linear quadratic model is the $\alpha / \beta$ ratio. From Eq.2.12 it can be seen that $\alpha / \beta$ ratio represents the dose at which linear and quadratic component have the same weight in the process of cell killing. This means that the number of cells killed by the two mechanisms, lethal lesion and sub-lethal lesion exchange, are equal. The $\alpha / \beta$ ratio can be determined from the fit of $L Q$ model to the survival curve, and from iso-effect analysis for fractionated regime where it acquires a notable importance.

For fractioned exposure LQ becomes:

$$
S=\exp \left(-\alpha D\left[1+\frac{d}{\alpha / \beta}\right]\right)
$$

where $D$ is the total dose in $\mathrm{n}$ fractions, $d$ is the dose per fraction $(D=n d)$ and $\alpha / \beta$ is the $\alpha / \beta$ ratio for one kind of tissue.

Equation 2.14 shows that for high values of $\alpha / \beta$ the main component of cell killing is non-repairable lesions expressed by the linear component. This makes survival strongly dependent on the total dose $D$ delivered to the tissue. However, if the dose per fraction $d$ is of same order as the $\alpha / \beta$ ratio, then both components have to be taken into account. This issue is strongly associated to the early and late cell-response as it is explained below. ${ }^{43}$ 
There is a cell cycle time dependence of the interval between irradiation and observable cellular response such as desquamation or reddening of the skin, losing the ability of forming colonies if the cells are growing in a petri dish, or organ reduced capacity. Early-responding cells are those that cycle quickly showing 'early' the damage; it could be in few days or weeks. For example skin, mucosa and most tumours are tissues that tend to have high values of $\alpha / \beta$ ratios. Late responding cells are those that cycle slowly showing the effect of radiation 'late', and they tend to have low $\alpha / \beta$ ratios. For example brain tissue, spinal cord, lung and kidney are late responding tissues and are the tissues that we want to protect in radiotherapy. The biological effect that relates the $\alpha / \beta$ ratio to the time required for the cells to divide and cycle is the strength of LQ approach. ${ }^{1,44}$

The mechanisms of cell killing in the tumours depend mainly on the total dose delivered, while for the normal surrounding tissue we have both components -the linear and the quadratic. Thus fractionation becomes an important strategy in order to control the tumour and spare the normal tissues.

After years of experience the efficacy of fractionation is well known. Based on radiobiological experiments and clinical data, it has been demonstrated that dividing a dose into a number of fractions spares normal tissues because the cells have time enough to repair sub-lethal damage between dose fractions, and they also repopulate if the overall time is sufficiently long. Thus, the LQ model 
explains one of the reasons why the therapeutic results are better for protracted irradiation than for a single dose.

\subsubsection{Shortcoming of LQ Model}

The derivation of $L Q$ model described in section 2.1.1 shows that the cell survival relationship has two components: a linear term $(\log S=-a D)$ and a quadratic term $\left(\log S=-\beta D^{2}\right)$. The initial slope is defined by the $\alpha$-component and the following region is described by a curve, which continuously bends as result of both components. In this model there is no exponential asymptote at high doses while many experimental cell survival curves exhibit a constant final slope. This has been a reason for criticism of the model regardless of its advantages.

Another method to derive a variant of the linear-quadratic formalism was developed by Carlone in order to investigate the ability of the model to properly describe the high dose region of survival curve. ${ }^{30,45}$

Other additional aspects to take into account are the adaptive and hypersensitive/radioresistant responses, which are presented in mammalian cells. ${ }^{46-48}$ There are many studies focused on the cellular and genetic damage after high dose exposure, however, much less is known about cellular response to low dose radiation. Cells respond in a unique manner to low dose radiation by activating or inactivating genes involved in damage repair mechanisms of chromosomes, membranes and proteins. ${ }^{49}$ It has been discovered that those 
genes involved with low dose radiation response are different from the ones responding at high doses.

Some studies in mice and human cells reveal an adaptive response when cells are treated with very small radiation dose allowing cells to easier withstand to higher dose, similar to cellular response at low-level damage from chemicals, bacteria or viruses. The data concerned with adaptive effects and presented by different laboratories disagree due to the differences in adaptive response for different cell-lines. ${ }^{50}$ In general this effect has been observed in small dose range up to 1 Gy for many cell-lines and it has been specially studied as an example of induced resistance in human lymphocytes. ${ }^{51}$

The next phenomena with potential interest in radiotherapy are the hyperradiosensitivity (HRS) and radioresistance (RR) presented also at very low doses. ${ }^{52}$ Typically there is a region with high sensitivity in the survival response at doses bellow 0.5 Gy and followed by a region of increased radioresistance beyond 0.5 Gy up to 1 Gy where it is maximal and the curve starts descending again. The molecular mechanism of the process is still not clear but the current understanding assumes that the HRS seems to be a common survival response at very low doses. The increment of the dose will raise the levels of radiationinduced damage above a damage-sensing threshold triggering protective response, which activates a more efficient DNA repair and a RR response. ${ }^{53}$ Even though RR is considered by some authors an adaptive response, it is still 
controversial whether the adaptive response mentioned before and HRS/RR are analogous phenomena and result from the same underlying mechanism. 


\section{Chapter 3}

\section{MATERIALS AND METHODS}

\subsection{Clonogenic Assays}

\subsubsection{Cell Lines and Culture}

Four different cell lines were investigated. First were normal and non human CHOAA8, hamster fibroblast cells because they are very easy to work with and they have been widely investigated. The next cases were different kinds of human tumour cells: U373MG, human glioblastoma cells; and human prostate carcinoma cell lines CP3, and DU-145. Especially, the choice of prostate cancer cells is based on the recent controversial results from clinical modeling proposing low values of $\alpha / \beta$ ratio. This issue is related to hypofractionation of prostate cancer in radiation oncology field. ${ }^{54-57}$ 
Cells were grown in a medium DMEM/F12 at $\mathrm{pH} 7$, temperature $37^{\circ} \mathrm{C}$ in watersaturated air containing $5 \% \quad \mathrm{CO}_{2}$. For Chinese hamster cells $1 \%$ penicillin/streptomycin antibiotic was added to avoid the risk of contamination.

\subsubsection{Irradiation Procedure}

Cells were irradiated using a Pantak Bipolar Series, model HF320 X-Ray unit operating at $250 \mathrm{kVp}$ with $1.87 \mathrm{~mm}$ base Aluminum filter and a customized Aluminum flattening filter giving a dose rate of $190 \mathrm{cGy} / \mathrm{min}$. All the doses were delivered in just one fraction 18 hours after the cells were plated.

\subsubsection{Clonogenic Survival}

The principle of survival in in vitro experiments is based as it was explained in section 1.2 upon the appearance of colonies, which stem from one surviving progenitor cell. A colony is defined as a group of cells surface attached and large enough to be recognized. It is usually assumed that the colony is composed of at least 50 cells. Depending on the expected surviving fraction after the irradiation treatment, different numbers of cells are plated into dishes or flasks. This is a very important step since if the surviving fraction is large and the flask is crowded with cells, they will not form colonies. On the other hand, if it is too small we have the risk of not having any surviving cells. This issue will be understood afterward when introducing the term called "plating efficiency" $(P E) .^{1,3}$ Usually, the number 
of plated cells is selected for the formation of 50 to 100 expected colonies after the irradiation.

Thus, if after a treatment the cell can retain its reproductive integrity and it is able to divide and multiply, then it will grow into a macroscopic colony that can be easily seen by eye and counted. These survivors are said to be clonogenic. However, even in the absence of treatment, not all the plated cells will grow and form colonies. Some cells might not divide or they might just multiply a few times, so they will not form visible colonies. This is a consequence of uncontrolled conditions related with growth medium, traumas associated with trypsinization and manipulation during the experiments. In addition, there are also uncertainties in counting cell suspension and preparing dilutions. That is why control cells are plated to determine the fractions of cells which will grow into colonies in absence of the treatment. The term is called plating efficiency and is defined as,

$$
P E=\frac{\text { colonies counted }}{\text { cells plated }} \quad \text { (No Irradiation) }
$$

Hence, the surviving fraction from irradiated cells is normalized to the PE to obtain the values just associated with irradiation process.

$$
F=\frac{\text { colonies counted }}{\text { cells plated } \times P E} \quad \text { (Irradiation) }
$$


The time required for the colony appearance depends on the growth properties of the different types of cells and the possible alterations of these properties by irradiation. In our experiment this time was taken to be 2 weeks, in which the colonies could be recognized for all cell lines. However, the size and spreading characteristics change with the cell type and dose.

The experiments were performed with cells from exponentially growing cultures, by initially seeding $3 \times 10^{5}$ cells in $10 \mathrm{ml}$ of media in T-75 flasks. When the exponential phase was reached the cells were trypsinized and plated in 4 or 10 $\mathrm{ml}$ of media in T-25 and T-75 flasks depending on the planned radiation dose. The dilutions were prepared to result in around 50 colonies after the irradiation procedure. Numbers of cells to be plated were calculated by using survival curves from similar cell lines and extrapolating to high dose using LQ and $L Q L^{45}$ models. We kept the cells in the incubator for $18 \mathrm{~h}$ before the irradiation and two weeks after the irradiation experiment the colonies were fixed, stained and counted. Six extra flasks were prepared for the control (0 Gy) to assess plating efficiency and for multiplicity determination.

Experiments were repeated three times for each cell line with irradiation given in $0.5 \mathrm{~Gy}$ increments and a total dose range varying from 0 Gy to 10.5 and 16 Gy for different cell lines. The survival was calculated as the average of these three experiments and the errors were estimated as the standard error of the mean. In each experiment the surviving fractions at different doses were the result of the 
average obtained from three flasks giving a total of nine contributions to every radiation dose point.

\subsubsection{Multiplicity Correction}

The correction for influence of cellular multiplicity is essential for the interpretation of survival response. ${ }^{3}$ Under propitious conditions, one colony usually represents one cell, which survived a particular treatment. However, if before treatment the cells have time to divide and reproduce, the assumed initial number of viable cells will change. The principle of survival does not distinguish between the original cells plated versus reproduced and just one colony will result from one, two or more proximal viable cells. Then, in order to avoid a colony formation after irradiation procedure in presence of multiplicity, it is necessary to kill all the cells in that colony.

The multiplicity factor is then considered the average value representing the multiplicity at times after plating and at the time of the radiation treatment. Thus, the survival curve, which is a relative measurement of viable cells after a treatment, needs to be corrected in order to account for the cell growth after plating.

Suppose that a cell A has been plated and there is no time for the cell to multiply before irradiation takes place. See scheme in Fig.3.1. In this case, assuming that 
$S$ is the probability of cell A to survive then $P_{K, A}=1-S$ is the probability of killing cell A.
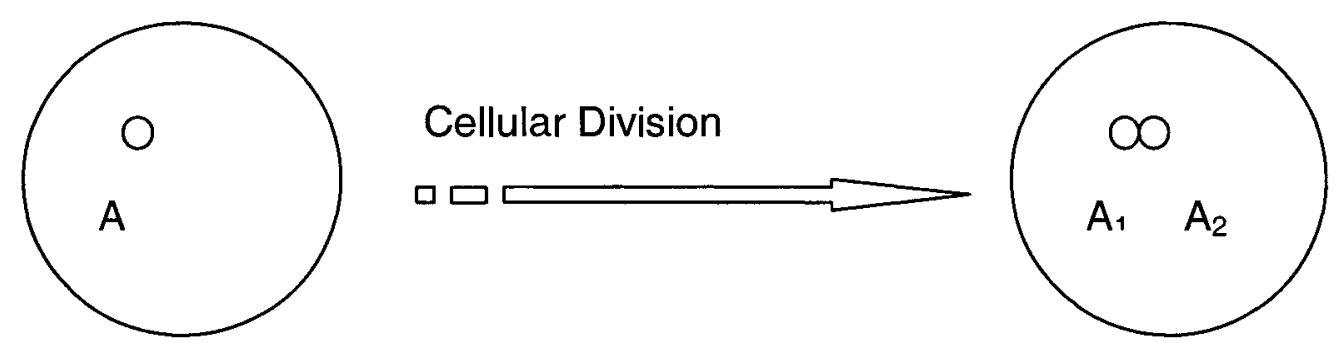

Fig.3.1 Schematic representation of a plated cell before and after cellular division with a multiplicity factor $\bar{N}=\mathbf{2}$.

However, if the cell has enough time to multiply before the treatment and assuming that the multiplicity factor $\bar{N}$ is equal 2 for example, then in order to say that the cell $A$ was killed, instead of killing one cell we need to kill two cells, $A_{1}$ and $A_{2}$. Now, the probability of killing $A$ is expressed by $P_{K, A}$ in Eq.3.3; and the real or corrected probability of cell survival is S.

$$
P_{K, A}=P_{K, A 1} P_{K, A 2}=(1-S)(1-S)=(1-S)^{2}
$$

For any value of the multiplicity factor $\bar{N}$, it becomes

$$
P_{K, A}=(1-S)^{\bar{N}}
$$

On the other hand, we know from the experiment that non-corrected survival fraction, $F$, is bigger than the actual value since at the time of irradiation there 
are more cells than the amount plated due to cellular division. Hence, the probability of cell killing, $P_{K, A}$, taken from the experiment is

$$
P_{K, A}=1-F
$$

Equating Eqs.3.4 and 3.5 we obtain,

$$
1-F=(1-S)^{\bar{N}}
$$

This means that for killing a plated cell in presence of cellular division, it is necessary to kill all cells stemming from cell division. Rearranging and solving for $\mathrm{S}$, the expression for corrected survival fraction is obtained:

$$
S=1-\exp \left[\frac{\ln (1-F)}{\bar{N}}\right]
$$

where $\bar{N}$ is the multiplicity factor; $\mathrm{F}$ is the survival fraction without correction and $S$ is the corrected survival fraction for the multiplicity.

\subsection{Data Analysis}

Experimental data are often accompanied by errors. That is why the resultant outcomes of the dependent variable vary even though the independent variables remain constant. Regression or curve fitting is the procedure carried out to estimate the trend of the outcomes. The fit of the raw experimental data to approximating equation or model is generally not unique for a given data set. 
Thus, the result of fitting process is to estimate the parameters of the model giving the minimal deviation from all data points. This can be obtained by least squares fitting method (LSM). ${ }^{58}$ Moreover, Monte Carlo technique is used to generate thousand of similar random experiments with the same statistics as the experiments. The simulated data are also fitted and the residuals analyzed.

The analyses were performed for different dose ranges defined by the arrows in Fig.3.2. The minimum range was limited to $4 \mathrm{~Gy}$.

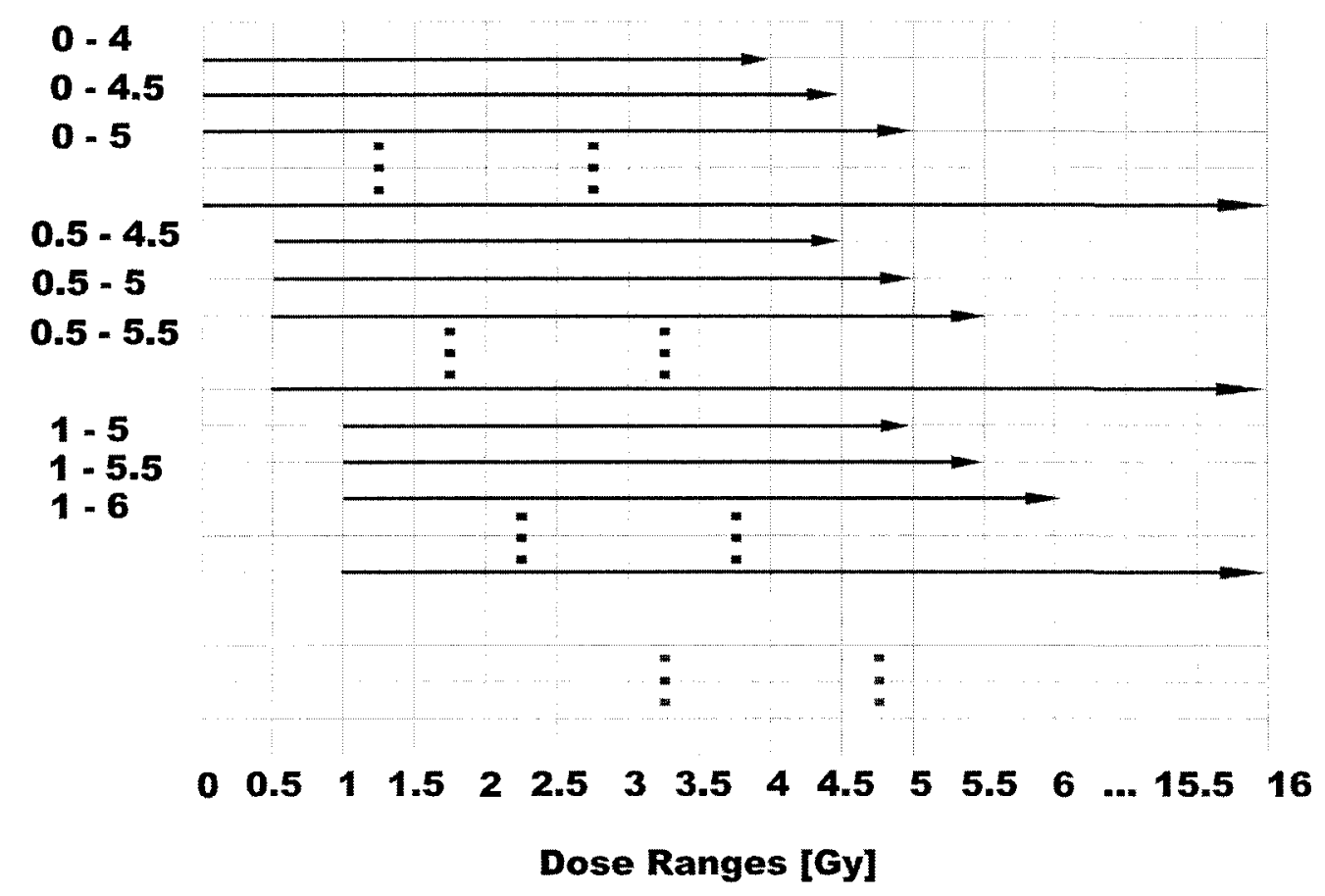

Fig.3.2 Dose ranges definition. Fixing the lower bound of the range at $0 \mathrm{~Gy}$, the upper bound changes every $0.5 \mathrm{~Gy}$, keeping at least $4 \mathrm{~Gy}$ as the minimal range. Next, the lower bound will move to the right also every $0.5 \mathrm{~Gy}$, each time repeating the same procedure described above.

First, we fixed the initial dose at 0 Gy and let the final dose vary with 0.5 Gy increments, keeping always at least 4 Gy as minimal range. Next, the initial dose 
is increased in 0.5 Gy and fixed while the final dose again varies with increments of $0.5 \mathrm{~Gy}$. These steps are repeated until the complete range of the experimental data was covered.

\subsubsection{Least Squares Fitting Method. Goodness of Fit}

The LSM obtains the coefficient estimates by minimizing the summed square of residuals. Assuming that the individual measurements $y_{i}$ are independent, the residual for the $\mathrm{i}^{\text {th }}$ data point is defined as the difference of the observed response $y_{i}$ and fitted response $\hat{y}_{i}$ :

$$
r_{i}=y_{i}-\hat{y}_{i}
$$

When each data point does not provide equally precise information, in other words, the response data do not have the same variance $\sigma$, weighted least squares regression can be used to improve the fit. Weighted least squares fitting maximizes the efficiency of parameter estimation giving more influence to those points with smaller variance and less influence to the points with higher variance. The summed square of residuals becomes

$$
S S E=\sum_{i=1}^{n} w_{i}\left(y_{i}-\hat{y}_{i}\right)^{2}
$$

where $\mathrm{n}$ is the number of data points and the weights are given by 


$$
w_{i}=\frac{1}{\sigma^{2}}
$$

Now suppose that the measured values at the ith point, $y_{i}, i=1,2, \ldots n$, have known errors $\sigma_{i}$. If $y_{i}$ is regarded as an independent Gaussian random variable centered about the true value $\lambda_{i}$, then $\varepsilon_{i}$ is also a normal distributed random variable with mean 0 and standard deviation $\sigma_{i}, \mathrm{~N}\left(0, \sigma_{i}\right)$.

$$
\begin{gathered}
\mathcal{E}_{i}=y_{i}-\lambda_{i} \\
E\left[\varepsilon_{i}\right]=0 \quad E\left[\mathcal{\varepsilon}_{i}^{2}\right]=V\left[\varepsilon_{i}\right]=\sigma^{2}
\end{gathered}
$$

The hypothesis test to specify $\lambda_{i}$ is based on the measurements. For example, the hypothesis $\mathrm{H}_{0}$ is represented by:

$$
\lambda_{i}=f_{i} \quad i=1,2, \ldots n
$$

If the hypothesis is true then the variable $u_{i}$ defined below is a standard normal distributed variable, $\mathrm{N}(0,1)$.

$$
u_{i}=\frac{y_{i}-f_{i}}{\sigma_{i}}
$$

Hence, variable $T$ given by Eq.3.14 will follow $x^{2}$-distribution with $n$ degrees of freedom. 


$$
T=\sum_{i=1}^{n} u_{i}^{2}=\sum\left(\frac{y_{i}-f_{i}}{\sigma_{i}}\right)^{2}=\chi^{2}
$$

Therefore, if the hypothesis is false the contribution of the deviation of each measurement from the values predicted by the hypothesis $\mathrm{H}_{0}$ and normalized by $\sigma_{i}$ will produce higher values of $\mathrm{T}$ than when the hypothesis is true. In other words, larger value of $x^{2}$ implicates larger discrepancy between the data and the hypothesis.

Since the expectation value of $a x^{2}$-distributed variable is equal to the number of degrees of freedom, df, the quantity $x^{2}$ divided by the number of degrees of freedom, $x^{2} / d f$, is used as a measure of goodness of fit. When $x^{2} / d f$ is much bigger than one, there is some reason to be uncertain about the hypothesis.

In order to reject the hypothesis it is necessary to establish the desired significance level $\alpha_{s l}$, which means the probability of having the chance of rejecting a true hypothesis. In the case of the test variable $T$, the significance level $\alpha_{\mathrm{sl}}$ is defined as:

$$
\alpha_{s l}=\int_{x_{1-\alpha}^{2}}^{\infty} f(z ; d f) d z
$$

where $f(z ; d f)$ is the $x^{2}$ - distribution for df degrees of freedom. Then, if $\mathrm{T}$ falls in the critical region defined by $T>x^{2}\left(1-\alpha_{s l}, d f\right)$, the hypothesis is rejected. It means 
that $T$ is bigger than the quantile $x^{2}\left(1-\alpha_{s 1}\right)$ of the $x^{2}$-distribution for df degrees of freedom.

The level of agreement between the observed measurements and the hypothesis can be tested as well determining the observed significance level, called P-value. In this case, the procedure is the other way around, instead of defining a significance level and finding the corresponding $x^{2}$-value for the distribution, now the significance level is determined using the same Eq.3.15, but in this case the integration is from the value of $x^{2}$ obtained from the fit until infinity. The P-value gives the probability of expected worse $x^{2}$ values than the one actually obtained. Very small observed significance level or P-value means that if the tested hypothesis were true, $\mathrm{x}^{2}$ as high or higher than the value already obtained by the fit would not likely be expected. Hence, the hypothesis could be safely rejected.

When the hypothesis tested is given by a function $\lambda\left(x_{i} ; \vec{\theta}\right)$ of independent variables $x_{i}$, with $k$ parameters $\vec{\theta}(1,2, \ldots k)$, the number of degrees of freedom is reduced. Certainly, if the estimated parameters are now functions of the measurements then the standard normal variables $u_{i}$ are no longer all independent. Therefore, the number of degrees of freedom is reduced by number of parameters giving equations of constrain (df- $k$ ). In that case Eq.3.14 becomes: 


$$
\chi^{2}(\vec{\theta})=\sum\left(\frac{y_{i}-\lambda\left(x_{i} ; \vec{\theta}\right)}{\sigma_{i}}\right)^{2}
$$

LSM does not assume a normal distribution of the errors when calculating the estimated parameters. The procedure is used even in those cases to estimate the parameters that give the minimum of summed square, as long as the measured variables $y_{i}$ are independent. However, the method works better for normal distributed errors and the results will coincide with Maximum Likelihood estimators. ${ }^{59}$ Moreover, statistical inference such as confidence and prediction bounds need normally distributed errors, expressed through Eq.3.12.

If the measurements are not independent, a more general expression for Eq.3.16 is represented by Eq.3.17, where $V$ is the known covariance matrix. ${ }^{59}$ This equation reduces to Eq.3.16 if the covariance matrix and consequently its inverse are diagonal which is the case for independent variables.

$$
\chi^{2}(\vec{\theta})=\sum_{i, j=1}^{n}\left(y_{i}-\lambda\left(x_{i} ; \vec{\theta}\right)\right)\left(V^{-1}\right)_{i j}\left(y_{j}-\lambda\left(x_{j} ; \vec{\theta}\right)\right)
$$

From Eq.3.11 we see that if the measured variable $y_{i}$ can be considered, as independent Gaussian distributed, then finding the true estimator $\lambda_{i}$ we will have random errors with mean equal zero. For that reason, when fitting normally distributed data to a model and the residuals given by Eq.3.8 do not have mean zero, then it might be that the model is not the right choice. 


\subsubsection{Parameter Estimation and Confidence Regions in LSM}

Although LSM could be applied to any kind of function $\lambda(x ; \vec{\theta})$ for simplicity and because the estimators obtained in that case have especially desirable properties, the analysis will be carried out for a linear function of the estimated parameters as shown in Eq.3.18. ${ }^{58}$

$$
\lambda(x ; \vec{\theta})=\sum_{j=1}^{k} a_{j}(x) \theta_{j}
$$

where $a_{j}(x)$ are linearly independent functions. For $i^{\text {th }}$ point it can be written as:

$$
\lambda\left(x_{i} ; \vec{\theta}\right)=\sum_{j=1}^{k} a_{j}\left(x_{i}\right) \theta_{j}=\sum_{j=1}^{k} A_{i j} \theta_{j}
$$

The Eq.3.17 in matrix notation becomes

$$
\chi^{2}=(\vec{y}-\vec{\lambda})^{T} V^{-1}(\vec{y}-\vec{\lambda})=(\vec{y}-A \vec{\theta})^{T} V^{-1}(\vec{y}-A \vec{\theta})
$$

where $\vec{y}=\left(y_{1}, \ldots y_{n}\right)$ is the vector of measured values and $\vec{\lambda}=\left(\lambda_{1}, \ldots \lambda_{n}\right)$ are the predicted values.

Calculating the first derivatives of $\mathrm{X}^{2}$ in Eq.3.20 with respect to $\theta_{i}$ and setting them to zero we can find its minimum,

$$
\nabla \chi^{2}=-2\left(A^{T} V^{-1} \vec{y}-A^{T} V^{-1} A \vec{\theta}\right)=0
$$


This can be solved for the estimators $\hat{\theta}$. A non-singular matrix $A^{\top} V^{-1} A$ must be provided.

$$
\hat{\theta}=\left(A^{T} V^{-1} A\right)^{-1} A^{T} V^{-1} \vec{y} \equiv B \vec{y}
$$

The solutions represented by the vector $\hat{\theta}$, are linear functions of the measurements $\vec{y}$ when $\lambda\left(x_{i} ; \vec{\theta}\right)$ is a linear function of the parameters $\vec{\theta}$.

The covariance matrix for the estimator $\mathrm{U}_{\mathrm{ij}}$ can be found using error propagation

$$
\begin{aligned}
& U_{i j}=\operatorname{cov}\left(\hat{\theta}_{i} ; \hat{\theta}_{j}\right) \\
& U=B V B^{T}=\left(A^{T} V^{-1} A\right)^{-1}
\end{aligned}
$$

and correspondingly the inverse covariance matrix, known as error matrix, is

$$
\left(U^{-1}\right)_{i j}=\frac{1}{2}\left[\frac{\partial^{2} \chi^{2}}{\partial \theta_{i} \partial \theta_{j}}\right]_{\vec{\theta}=\hat{\theta}}
$$

There are different ways of estimating the confidence region of the fitted parameters: analytical, graphical or using Monte Carlo simulation among others. ${ }^{58}$ Confidence region can be seen as parameter space reflecting its statistical uncertainty. The analytical method consists of determining the second derivatives with respect to the parameters, which for not very complicated functions can be done numerically. Then, calculate the error matrix as in Eq.3.25; 
next, invert it to obtain the covariance matrix, whose diagonal elements represent the variance of the fitted parameters. The square roots of the diagonal elements define the symmetric confidence limits or sometime called symmetric errors. ${ }^{59}$

If the function $\lambda(x ; \vec{\theta})$ is not a linear function of the parameters $\vec{\theta}$ we can still relate it to a linear case situation expanding the function in Taylor series about the initial value of the vector $\vec{\theta}$ obtained in another way. Then, keeping just the first term we get exactly what we have in Eq.3.20.

Thus, in the nonlinear case our considerations about LSM remain similar to the linear case. However, for the convergence of the method a good first approximation is needed. Certainly, if within the region between the first approximation and the solution, the first term truncated Taylor series represent a good approximation, then the probability of convergence will increase. It means that the approximation works better when the nonlinear deviations during the iteration process are small in the region where the parameters vary and also when the variations of the parameters are small.

The covariance matrix in nonlinear case also has the same interpretation as before, especially, in the case of small measurement errors. This is due to the fact, that propagating small original errors, we will have small errors for the fitted parameters. 


\subsubsection{Introduction to Monte Carlo Method}

The Monte Carlo method is a technique, which provides approximate solutions to a variety of mathematical and physical problems by performing statistical sampling experiments on a computer. The method applies to problems with no probabilistic content as well as to those with inherent probabilistic structure. The method is based on calculation of probabilities and other related quantities by using random numbers.

A Polish born mathematician, Stanislaw Ulam, invented Monte Carlo method. He worked for John von Neumann on the United States' Manhattan Project during World War II and he is known for designing the hydrogen bomb with Edward Teller in 1951. Ulam invented the Monte Carlo method in 1946 while pondering the probabilities of winning a card game of solitaire. ${ }^{60}$

However, Monte Carlo methods were actually first used as a research tool during the Second World War for the developing of the atomic bomb. It was applied to simulate probabilistic problems related to neutron diffusion in fissile material. Along with computer progress Monte Carlo methods have been extensively applied in the past decades. Some of them are mentioned below:

- Evaluation of integrals including path integrals

- Integral equations

- Boundary value problems for Partial Differential Equations 
- Ordinary differential equations with random entries

- Ordinary stochastic differential equations

- Simulation of random variables, stochastic processes and fields

Those implementations are applied to different fields of science. One of the areas as we mentioned is the use of Monte Carlo method for simulation. The technique not only could be used to simulate processes that follow statistical laws, but also measurements and its errors.

\subsubsection{Monte Carlo Method for Simulation. Modeling}

In modeling, Monte Carlo analysis has been widely used to provide accurate and reliable statistics for the parameters of a model fitting experimental data. The problem with fitting is that the parameters obtained have a finite uncertainty value, which is unknown, but necessary in order to interpret the confidence one may have about the fitted results. However, some conditions have to be accomplished. We must have a reliable data that can be considered Gaussian distributed and the model has to successfully fit the data in order to have Gaussian distributed residuals with mean zero and the same variance as the original data.

In these conditions, the solution from the initial best-fit parameters will produce a certain variance, which reflects the error level in the data. Using a random 
number generator, you regenerate random errors, which have the same properties as the error in the original data, i.e., they are random, and have a Gaussian distribution with the same standard deviation (or variance) as the original dataset. Then you add this error distribution to your best-fit solution (the fit) and you have an equivalent experimental data set that is slightly different from the original dataset, and can be refitted. The Monte Carlo analysis can be performed repeating this procedure thousands of times to accurately determine the uncertainties contained in the parameter estimates and test goodness of fit from the actual distribution of $x^{2}$ variable. Thus, instead of repeating complicated experiments many times, which could be very time consuming, expensive or sometimes impossible, they are simulated.

One of the reasons for not having normally distributed residual with mean zero, assuming normality of the data, as was explained before is that the model does not properly describe the data. In this study, the Monte Carlo technique is applied, similar to what was explained above, to repeatedly simulate random data sets based on statistics of the measured data. However, we do not have a reliable model and what we want to determine is the correspondence of $L Q$ model with the experimental data for different ranges of doses.

The measured survival fraction is assumed to be a normally distributed variable since the clonogenic survival assay is a technique involving many different kinds of errors. According to the central limit theorem the total error will be Gaussian 
distributed if the error may be regarded as the sum of large number of small contributions. Hence, as is shown in Eq.3.11 a measured value at $\mathrm{i}^{\text {th }}$ point $y_{i}$, is regarded as a Gaussian random variable centered about the true value $\lambda_{i}$. Therefore, if the form of the hypothesis is correct, which means that we chose the right model, it would be expected to have: random residuals normally distributed with mean zero, $\mathrm{N}\left(0 ; \sigma_{i}\right) ; \mathrm{X}^{2}$-distribution of the variable $\mathrm{T}$ in Eq.3.16; and flat distribution for the P-values. Otherwise, if the hypothesis were incorrect, the mentioned distributions would be far from the expected.

The steps followed to implement Monte Carlo simulation of random input data are:

- In order to have a reliable data set, because the simulation is based on the statistics of measured data, three experiments were performed. Each of them is the result of three more experiments; in total we have nine contributions to the average survival fraction, whose error is represented as the standard error of the mean. This was carried out for a large range of different doses, as explained in Clonogenic Assays section.

- At each dose random Gaussian error is added to the magnitude of survival fraction for all measurements. The error is based on the mean value obtained from the average during the measurement process.

- The LQ model fit is applied and $x^{2}$ and P-values are identified and saved.

- The simulation was repeated 1000 times and the distributions were plotted. 
This is basically a generation of normally distributed variable with the mean equal to average survival fraction and standard deviation from the standard error of the mean for each dose point. ${ }^{61}$

The T variable in Eq.3.16 becomes in Monte Carlo experiments in Eq.3.26 where $\lambda_{i}\left(\hat{\theta}_{1} ; \hat{\theta}_{2}\right)$ is a model of two parameters $\hat{\theta}_{1}, \hat{\theta}_{2}$, resulting from the LQ fit of the $i^{\text {th }} \mathrm{MC}$ data.

$$
\chi^{2}=\sum\left[\frac{M C\left(y_{i} ; \sigma_{i}\right)-\lambda_{i}\left(\hat{\theta}_{1} ; \hat{\theta}_{2}\right)}{\sigma_{i}}\right]^{2}
$$

In this way, the procedure simulates repeated identical experiments on the same sample, and for each simulation the data is fit and the outcomes determined. This Monte Carlo technique is not the ideal because the method cannot account for the bias introduced during the estimation of the survival fraction. However, we do not know if the hypothesis of LQ model is the correct or not, thus, this is what we want to verify and the statistics of the measured data are the closest to the reality that we have. ${ }^{61}$ In addition, it is important to note that if the form of the hypothesis is not correct, then a small statistical error in the parameter is not sufficient to imply small uncertainty in the estimates. 


\section{Chapter 4}

\section{RESULTS AND DISCUSSION}

The results presented in this chapter are based on the methods described in Chapter 3. First of all, detailed data sets were obtained for the cell lines studied and the importance of inclusion of a multiplicity factor is shown. The broad dose range of survival curves allowed a more complete analysis of the agreement between LQ approach and experimental survival, which has not been done before. The results are founded on the $\chi^{2} / d f$ statistics from the fit of the LQ model to experimental surviving fraction for different dose ranges. In addition, a thousand experiments were generated applying Monte Carlo technique to estimate the difference of our ' $X$ ' and 'P-value' distributions under the hypothesis that LQ model properly describes the survival and assuming that errors are standard normal distributed. 


\subsection{Multiplicity Correction}

A comparison between cell survival measured by the clonogenic assay with and without taking into account the multiplicity factor for U373MG cell line is shown in Fig.4.1.

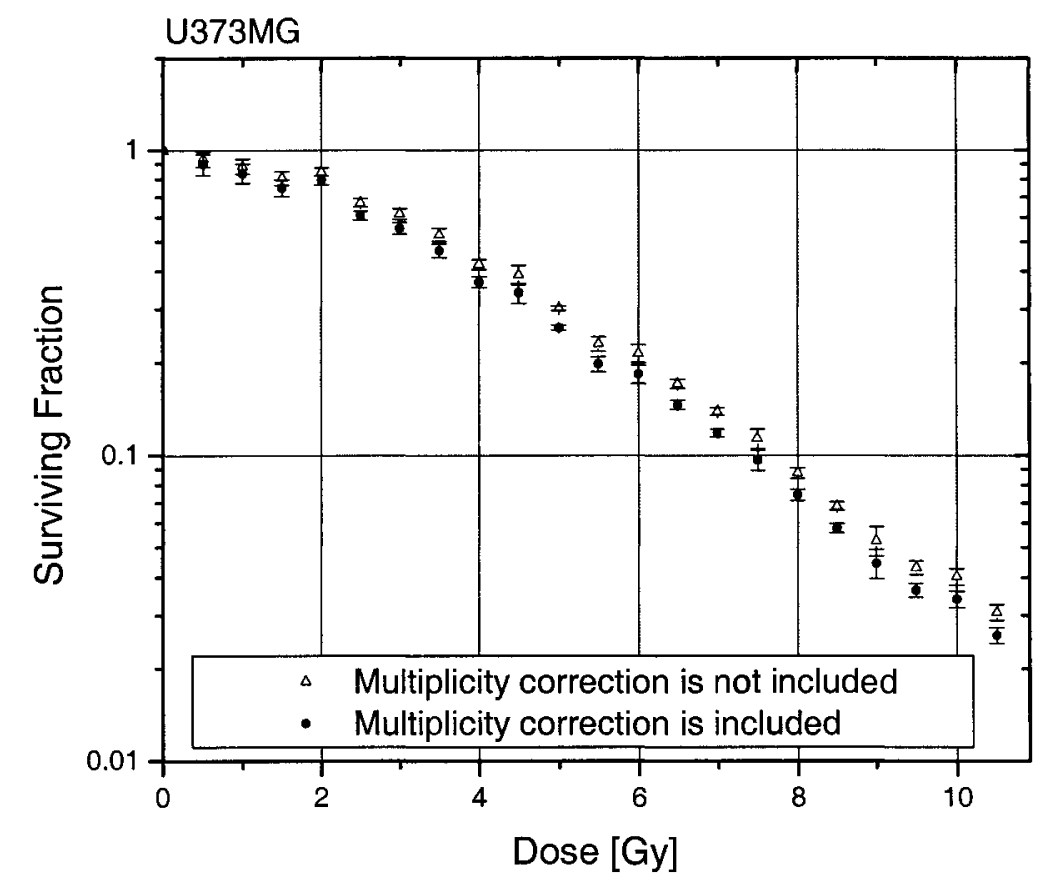

Fig. 4.1 Survival Curve for U373MG cell line from clonogenic assay.

The multiplicity factors for the studied cell lines are shown in the Table 4.1. The table shows that in $18 \mathrm{~h}$ all the cells studied have time enough to replicate and the cellular division is a faster process in the CHOAA8 cell line compared to the 
other cell lines. Even for the case of U373MG, which reflects the lower multiplicity factor, there is an evident influence in survival response as observed in Fig.4.1.

Table 4.1 Multiplicity factor for studied cell lines

\begin{tabular}{|l|c|c|}
\hline Cell line & Multiplicity Factor & Standard Deviation \\
\hline CHOAA8 & 2.2833 & 0.1142 \\
\hline U373MG & 1.1891 & 0.0144 \\
\hline CP3 & 1.2670 & 0.0876 \\
\hline DU145 & 1.2372 & 0.0558 \\
\hline
\end{tabular}

Since $18 \mathrm{~h}$ is a long enough period for cells to reproduce and all cell lines show an influence of multiplicity factor on survival, we corrected the data before any further analysis.

\subsection{Experimental Survival Curves}

The clonogenic survival curves were determined as defined in Eq.3.2. As we said before, each data point is the average of three independent experiments and error bars represent the standard error of the mean.

The quality of the data obtained from the clonogenic assays varies for the different cell lines. The process of colony formation depends on the growth properties and the adaptability to in vitro conditions of each cell line. In addition, these properties will also change with different radiation dose. The size, shape 
and characteristics of the colonies are altered by the ionizing radiation. The colonies in the control flasks are fairly uniform in size, about $2-3 \mathrm{~mm}$, while the irradiated flasks display colonies of different sizes, some of them barely visible. Moreover, while the control colonies are compact the irradiated colonies are very dispersed with vacant areas due to cell detachment and 'giant' cells formation. The Fig.4.2 shows the stained colonies obtained in the clonogenic assay for CHOAA8 and CP3 cell lines. 


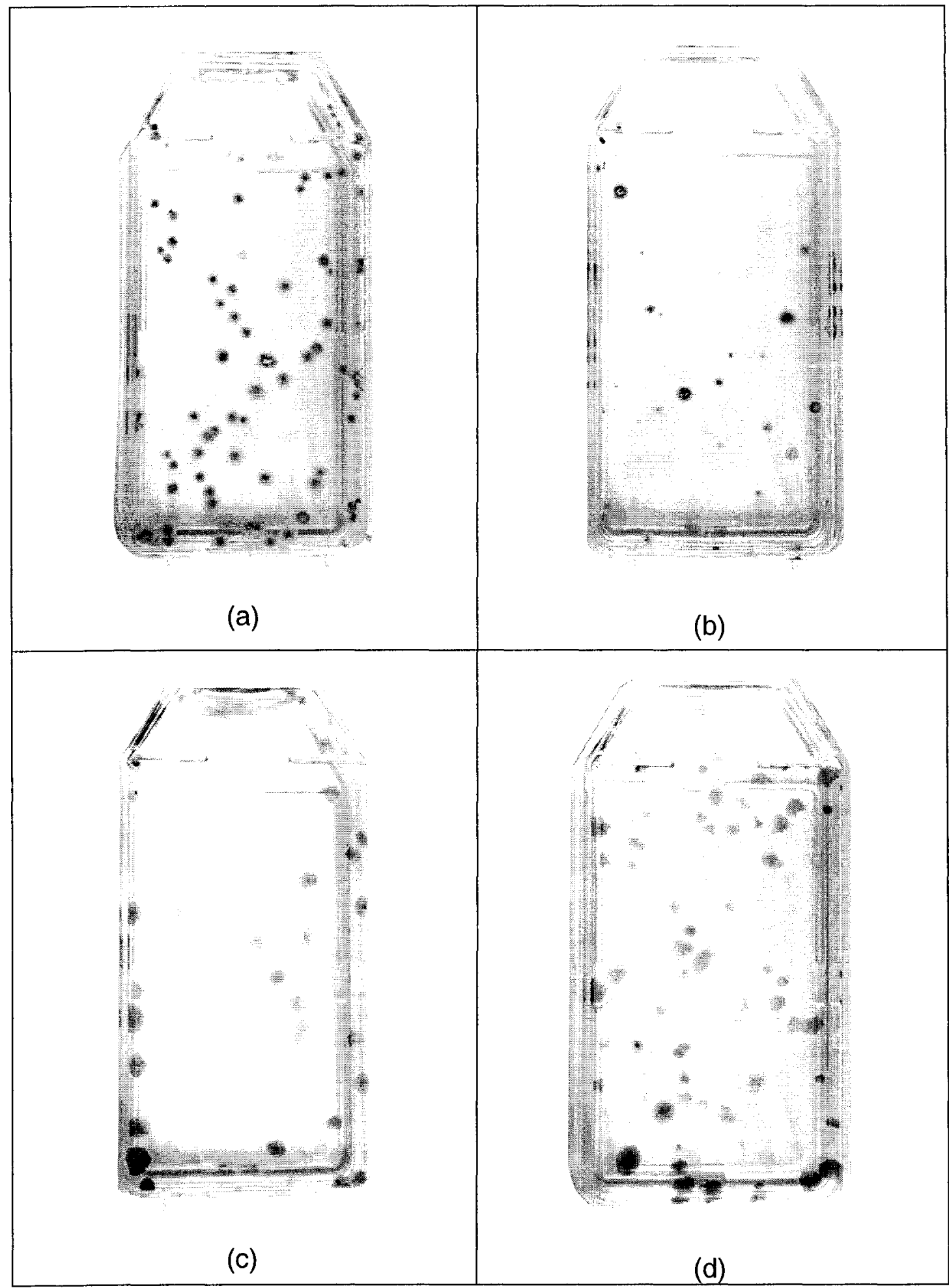

Fig.4.2 Stained colonies. (a) Control for CHOAA8, (b) High dose for CHOAA8, (c) Control for CP3, (d) High dose for CP3. 


\subsubsection{Hamster fibroblast survival curve}

The Fig.4.3 shows clonogenic survival curve of CHOAA8 hamster fibroblast cells within a range of 0-16 Gy, with an increment of $0.5 \mathrm{~Gy}$, which gives in total 33 data points for the total range.

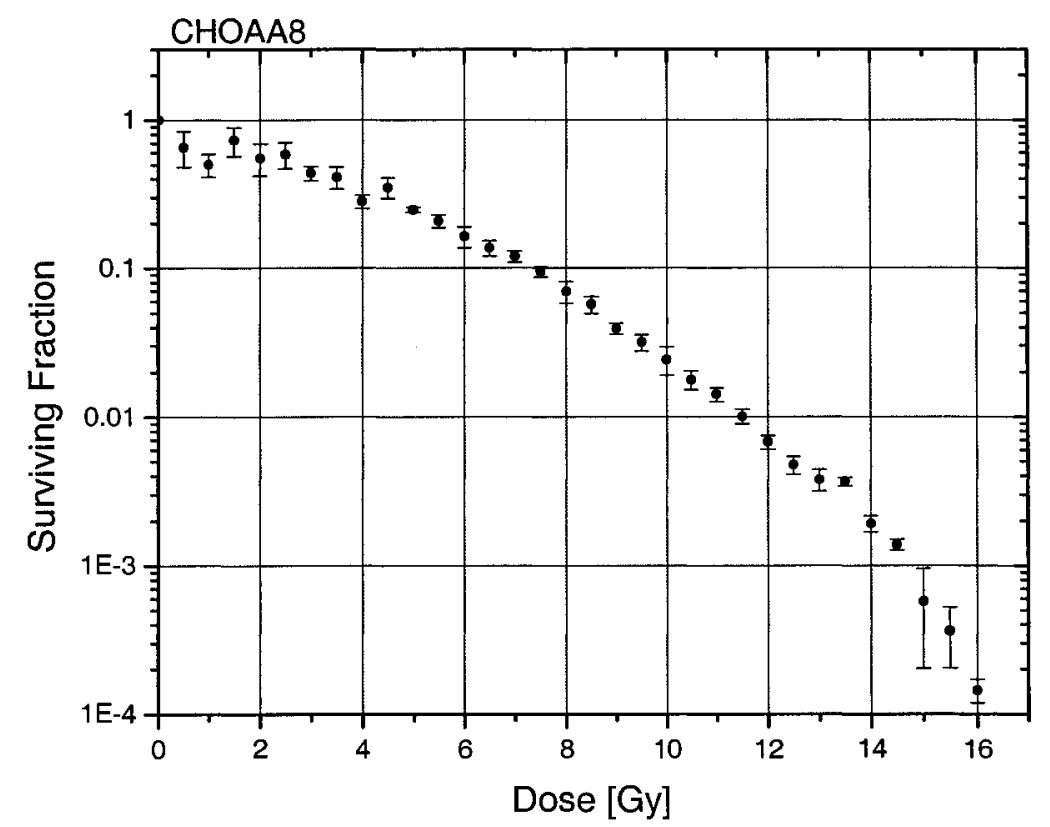

Fig.4.3 Survival Curve for CHOAA8 cell line from clonogenic assay.

This graph shows that survival levels of $10^{-4}$ were reached. However, it has huge error bars for the points with large doses compared to the low dose values. Relative errors of 65,44 and $18 \%$ were obtained in the last three data points. Thus, for these particular data small values of survival were obtained but they greatly fluctuate because of the large uncertainties. The inaccuracy of clonogenic 
survival technique at high doses due to the difficulty of the colony counting procedure, and colony shape and size variability is the reason of survival level limitation.

Errors a bit bigger than $20 \%$ were also obtained for the low dose range $(\leq 2.5$ Gy). Nonetheless, the survival curve reflects an increase of radiosensitivity for very low doses, less than $\sim 1$ Gy followed by an increase of the cell line radioresistance at 1.5 Gy where the curve starts bending downward in its expected trend.

\subsubsection{Human glioma cell survival curve}

The clonogenic survival curve for U373MG glioblastoma cells was shown in Fig.4.1 The data with a total number of 22 data points reached a surviving fraction of 0.026 at dose of 10.5 Gy. The uncertainties are more stable throughout the curve compared to CHOAA8 cell line with an average relative error of $5 \%$. There is just one data point with error bigger than $10 \%$ at 9 Gy with a relative error of $10.98 \%$. The curve as in the earlier case seems to display hypersensitive effects at doses below $\sim 1.5$ Gy followed by an increase of the surviving fraction at 2 Gy. 
Even though the uncertainties in this experiment are small, the hypersensitivity/radioresistance effect is small compared to the effect seen in the CHOAA8 cell line.

\subsubsection{Human prostate cancer cell survival curve. $\mathrm{CP} 3$ cell line.}

The prostate carcinoma cell line, CP3, displays more radiosensitivity compared to the other studied cell lines. A total number of 29 data points were obtained and they are shown in Fig.4.4. With the clonogenic assay the lowest surviving fraction reached is $3.5 \times 10^{-5}$ at dose of $14 \mathrm{~Gy}$. The clonogenic assay for CP3 cell line was more complicated compared to the cell lines above. This cell line is hard to work with and the limitations of the technique, related to low survival levels are emphasized. The survival curve displays unsteady uncertainties along the whole dose range. In the first 7 Gy the relative errors are much less than $20 \%$ with an average of $12 \%$. At higher doses the errors fluctuate extremely with relative errors within a range between 8 and $46 \%$. In addition, this cell line exhibits an increase of the survival curve at doses below $\sim 1$ Gy which could be considered an adaptive response at low doses. 


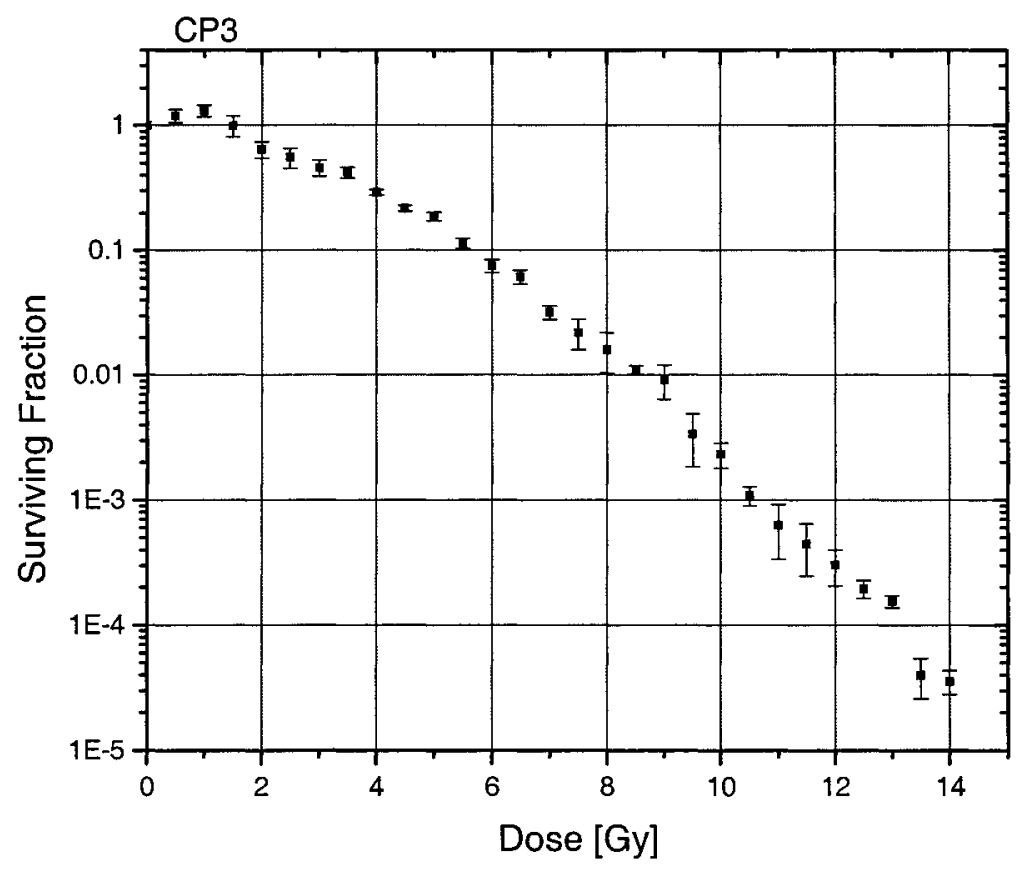

Fig.4.4 Survival Curve for CP3 cell line from clonogenic assay.

\subsubsection{Human prostate cancer cell survival curve. DU145 cell line}

The prostate carcinoma cell line, DU145, exhibited more radioresistance than the other prostate carcinoma cell line studied. The data obtained consist in 29 points with a maximal dose of 14 Gy (See Fig.4.5). The minimal surviving fraction 
reached is 0.0045 . Although the error bars are not large with an average relative error of $5.4 \%$, there are fluctuations in the data and the magnitude of the uncertainties and even the errors seems to be underestimated.

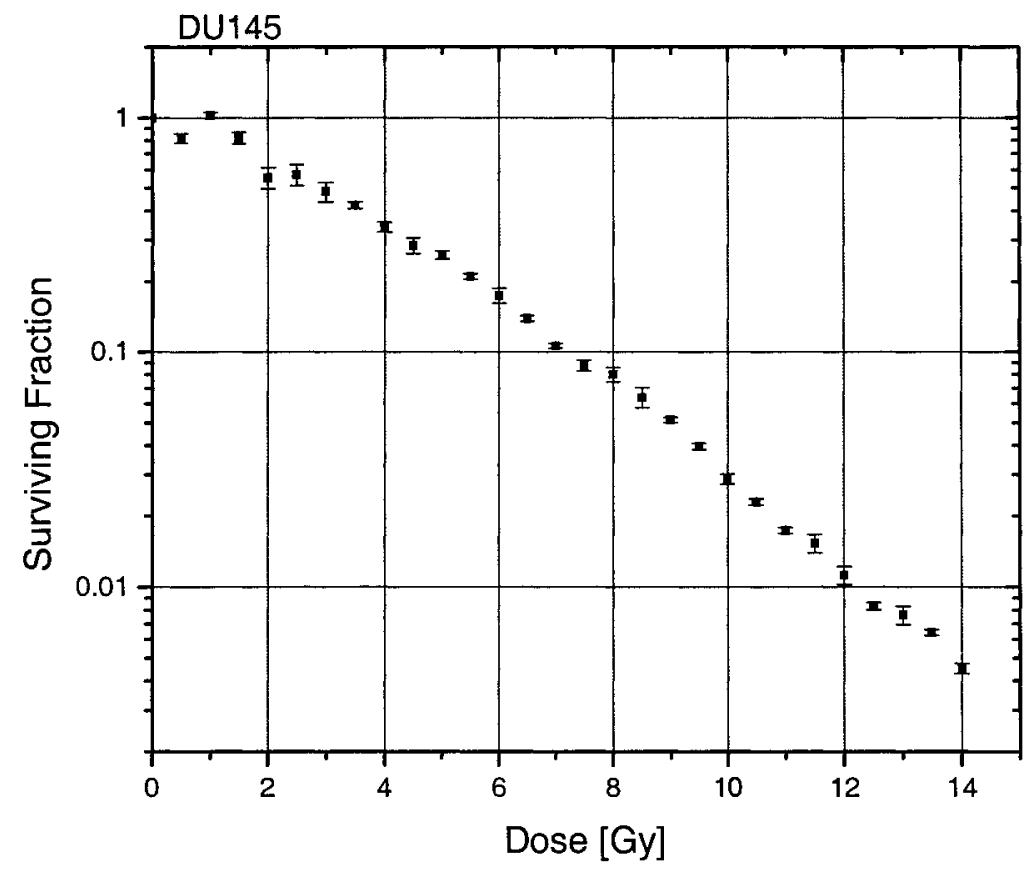

Fig.4.5 Survival Curve for DU145 cell line from clonogenic assay. 


\subsubsection{Discussion}

\subsubsection{Survival Curve Uncertainties}

The clonogenic cell survival assay is a widely applied technique used to determine the cellular response to ionizing radiation. The clonal growth of the cells in tissue culture is technically difficult, especially for human tumour cells because of their often low growth rate and particular growth requirements. On the other hand, the clonogenic survival assay technique itself involves several procedures carrying large uncertainties and as mentioned before the cell adaptation and response in in vitro conditions vary from one cell line to another.

Certainly, there are many factors concerned with the sources of the uncertainties. There are alterations in the media and solutions employed in the clonogenic assays, such as: growth medium, trypsin used to detach cells from the surface, and isotonic solution used to wash the cells.

There are also uncertainties related with manipulation during single-cell suspension and dilution preparations and counting. In addition, there are inexactnesses in time, temperature and oxygen concentration during incubation. Moreover, there are inaccuracies in the irradiation procedure and the colony counting process is very imprecise due to the variability in shape and definition of the colonies for different cell lines and applied doses. 
In practice, even though we try to keep all these conditions constant, they still have some inaccuracies, as well as the cellular response of different cell lines to these conditions differ. That is why there is variability in the exactness among different clonogenic survival assays and for different dose ranges. CHOAA8 and CP3 cell lines exhibited larger uncertainties compared to U373MG and DU145. In addition to what was explained before, there are other factors that seem to be involved with the lack of precision for these cell lines.

The number of surviving colonies obtained in CHOAA8 at very high doses was larger than the expected having more than 200 of colonies in the flasks. This made the process of colony counting very difficult increasing the uncertainties. Sometimes the colonies overlap or a few surviving cells will grow in just one colony. The data published until now did not go beyond 10 Gy of dose. Therefore, we had to extrapolate from known models to approximately determine the expected surviving fraction in order to have a reasonable number of surviving cells, able to grow and form colonies. However, as we know, the models do not always properly work at very high doses.

For the CP3 cell line, we have an increase of the uncertainties from about 7 Gy. In this assay, there were two main problems that seem to be related to the increase of the error bars. One was the low plating efficiency and the other one was that the fixed and stained colonies were very light. In general, CP3 cells do not easily adapt to in vitro conditions. Thus, we had a very low number of 
surviving colonies, making the statistics very poor especially in the mentioned region where the surviving fraction becomes smaller.

Comparing with similar published data from other clonogenic survival assays and presented in section 1.2 for CHOAA8, CP3 and DU145 cell lines, the uncertainties in the performed experiments seem to be comparable. This is even praiseworthy if we take into account that the survival curves were determined in a very extensive dose range where very small values of surviving fraction were obtained and the clonogenic survival technique is very difficult. Table 4.2 displays a comparison of the level of uncertainties from different clonogenic survival assays for the same group of cell lines investigated in this study. The first row shows the maximal dose used in the obtaining of the survival curve for both our experiment and data published by other authors. ${ }^{25,26,62}$ The second row displays the average of the relative error in percentage, over the total dose range studied and the last row the range of its variability.

Table 4.2 Comparison of uncertainties obtained from different clonogenic survival assays. Our experiments and other similar data are included.

\begin{tabular}{|l|c|c|c|c|c|c|c|c|}
\hline \multirow{2}{*}{ Experiments } & \multicolumn{2}{|c|}{ CHO Data } & \multicolumn{2}{c|}{ CP3 Data } & \multicolumn{2}{c|}{ DU145 Data } & \multicolumn{2}{c|}{ U373 Data } \\
\cline { 2 - 9 } & $\begin{array}{c}\text { Published } \\
\text { Ref. 26 }\end{array}$ & $\begin{array}{c}\text { This } \\
\text { work }\end{array}$ & $\begin{array}{c}\text { Published } \\
\text { Ref. 25 }\end{array}$ & $\begin{array}{c}\text { This } \\
\text { work }\end{array}$ & $\begin{array}{c}\text { Published } \\
\text { Ref. 25 }\end{array}$ & $\begin{array}{c}\text { This } \\
\text { work }\end{array}$ & $\begin{array}{c}\text { Published } \\
\text { Ref. 62 }\end{array}$ & $\begin{array}{c}\text { This } \\
\text { work }\end{array}$ \\
\hline Maximal Dose [Gy] & $6-10$ & 16 & $6-10$ & 14 & $6-10$ & 14 & 8 & 10.5 \\
\hline Ave. Rel. Err. [\%] & 13 & 16 & 16 & 20 & 25 & 5 & 22 & 5 \\
\hline Rel. Err. Range [\%] & $8-19$ & $4-65$ & $15-20$ & $5-46$ & $12-43$ & $1-10$ & $4-54$ & $2-10$ \\
\hline
\end{tabular}




\subsubsection{Survival Curves. General Trend}

There are a few features that can be discussed from the survival curve behaviour, all of them related to the cell radiosensitivity, which change along different dose ranges due to different cellular mechanism of response.

The range of radiosensitivity varies among several cell lines. We have studied four cell lines, three of them are human tumour cell lines (U373MG, CP3, DU145) and the other one is a hamster normal cell line (CHOAA8). Even though the maximal dose reached in the survival curves is different, Figs. 4.1, 4.3, 4.4 and 4.5 illustrate that the range of radiosensitivity is diverse for the cell lines analyzed.

The low dose region up to around 5 Gy is very important from a mechanistic and practical point of view. The LQ model includes a log linear component of cell inactivation and the dose per daily fraction in clinical radiotherapy are also often in the region of $2 \mathrm{~Gy}$. The correlation of the initial slope of cell survival with clinical response with radiotherapy has made radiosensitivity an aspect of interest. This initial linear behavior is not always clearly seen in these experiments, because it is hidden behind some hypersensitive and adaptive responses, or large uncertainties. However, the survival curves for CHOAA8, CP3 and U373MG still show a linear trend in about the first 4 Gy of dose. This issue will be analyzed in detail later on. The initial slope could be evaluated either 
by the parameter $\alpha$ of $L Q$ model, which we will see later or by the simple procedure of reading off the surviving fraction at dose of $2 \mathrm{~Gy}$.

Table 4.3 summarizes the degree of radiosensitivity among those cell lines already seen in the Figs. 4.1, 4.3, 4.4 and 4.5. First, the surviving fraction and the standard error of the mean at 2 Gy are shown, which is a measure of the initial slope of the survival curve. Next, the same is shown for 10 Gy to compare the level of responsiveness at higher doses. At low doses U373MG seems to be less radiosensitive compared to the other cell lines. The survival response at high doses was more sensitive for CP3 cell line.

Table 4.3 Surviving fractions and standard error of the mean from clonogenic survival for several cell lines at doses of $2 \mathrm{~Gy}$ and $10 \mathrm{~Gy}$.

\begin{tabular}{|l|c|c|c|c|}
\hline \multirow{2}{*}{ Experiments } & \multicolumn{2}{|c|}{2 Gy } & \multicolumn{2}{c|}{10 Gy } \\
\cline { 2 - 5 } & SF2 & SEM2 & SF10 & SEM10 \\
\hline CHOAA8 & 0.552 & 0.134 & 0.024 & 0.005 \\
\hline U373MG & 0.797 & 0.029 & 0.034 & 0.002 \\
\hline DU145 & 0.554 & 0.057 & 0.029 & 0.001 \\
\hline CP3 & 0.643 & 0.095 & 0.002 & 0.0005 \\
\hline
\end{tabular}




\subsection{Data Analysis Results}

This study is focused on finding the degree of correspondence between the survival curve measurements and the LQ model in different dose ranges. That is why the analysis is centered on parametric fitting of the data to the $L Q$ formalism. The methodological scheme used was presented in the Fig.3.2. The data were fitted changing the initial and final values of the range every 0.5 Gy and keeping at least 4 Gy as the minimal range such that all possible ranges were covered. First, we fixed the initial dose and let the final dose vary. In this case we are looking for the influence of including high dose data. Second, we fixed the final dose and the initial dose varies seeing the influence of low dose points in the LQ fit. Thus, every time the dose range is changed the data are fitted and the least square estimators for $L Q$ formalism and $x^{2}$-statistics are obtained. In addition, " $\mathrm{X}$ " and P-value distributions are also procured from Monte Carlo simulation of the experiments for the same determined ranges. The results for each cell line are presented in the next sections. 


\subsubsection{Results of data analysis for CHOAA8 cell line}

\subsubsection{Evaluating goodness of fit for CHOAA8 cell line}

Chi-square statistics divided by the number of degree of freedom $\left(x^{2} / \mathrm{df}\right)$ from the LQ fit are plotted for different dose ranges for CHOAA8 cell line. Figure 4.6 shows a few cases fixing the initial dose at $0,2.5$ and 5.5 Gy while the final dose varies along the $\mathrm{X}$-axis of the graph.

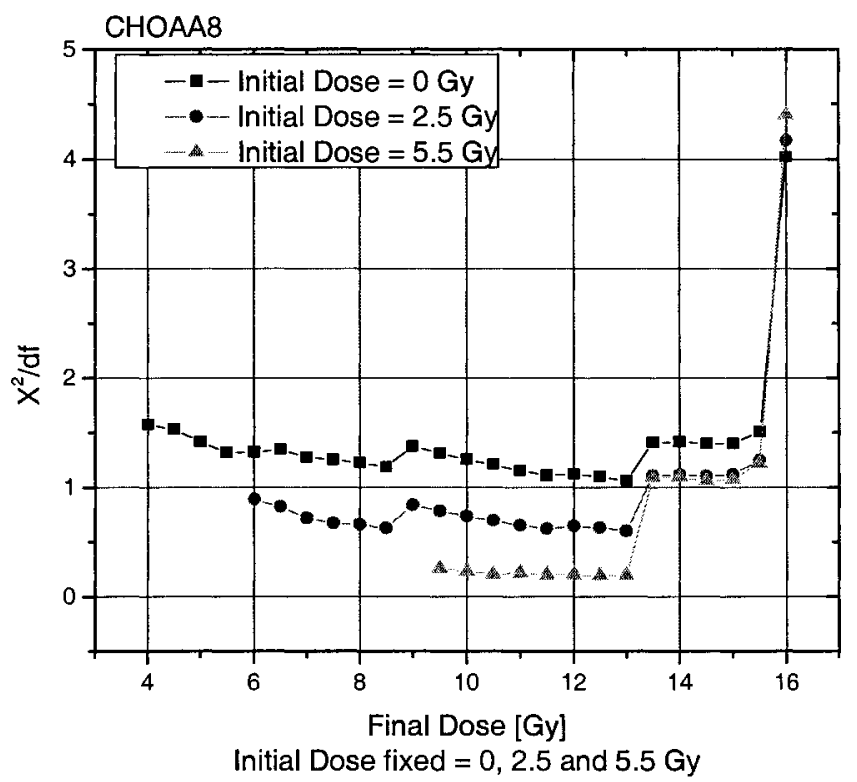

Fig. 4.6 Chi-Square divided by the number of degrees of freedom at different dose ranges for CHOAA8 cell line. Initial dose of the range is fixed at 0, 2.5 and 5.5 Gy. Final dose of the range varies along the $X$-axis.

The figures show that there is variability of $x^{2} / d f$ depending on dose range used in the fit. From Fig.4.6 we can see that at some point for high doses the fit 
worsens defining a clear threshold around 13 Gy, which becomes more evident when the low dose data are not used. This is shown in the graph by the data with triangles, when the initial dose of the range fitted is 5.5 Gy. Another large step in all the curves is seen at 15.5 Gy displaying a substantial disagreement between LQ model and the data with $x^{2} / d f>4$. In addition, there are two shifts observed when the data points below 2.5 Gy and 5.5 Gy are excluded. Hence, the same analysis is carried out for fixing final dose while changing the initial dose.

Even though the analysis was done for any final dose, Fig. 4.7 displays the case when the final dose was fixed at $13 \mathrm{~Gy}$, because it seems that higher doses do not improve the fit. Thus, the harmful impact of high dose data is eliminated which allows seeing a clearer picture of the low dose data influence.

The figure shows three well defined levels with mean values of the $x^{2} / d f$ statistics around 1.08, at very low doses, 0.61 between 1.5 and 4 Gy, and 0.20 for final dose higher than 4 Gy. The analysis reflects an improvement of the fit around $43 \%$ just excluding the range $\leq 1 \mathrm{~Gy}$. If the low dose data up to $4 \mathrm{~Gy}$ are not included $x^{2} /$ df drops in total $81 \%$. 


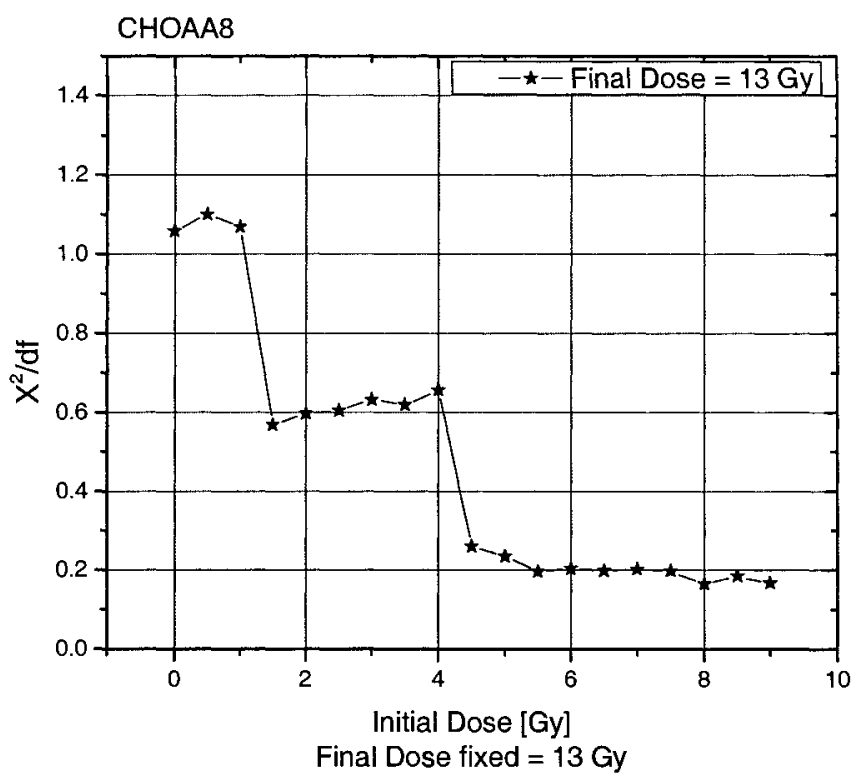

Fig. 4.7 Chi-Square divided by the number of degrees of freedom at different dose ranges for CHOAA8. Final dose of the range is fixed at $13 \mathrm{~Gy}$. Initial dose of the range varies along $X$ axis.

On the other hand, assuming that the measured values are independent and normally distributed, and the errors are known and standard normal distributed, as explained in section 3.2 .1 ; it is possible to test the hypothesis that the LQ model describes the data $\left(H_{0}\right.$ : LQ model). For that, we chose a significance level of $5 \%$ and the results for several ranges are shown in Table 4.4 . 
Table 4.4 $\mathrm{X}^{2}$-Test for CHOAA8 cell line at several dose ranges. Testing the LQ hypothesis for a significance level of $5 \%$. In the last column the P-value is also shown.

\begin{tabular}{|l|c|c|c|l|c|}
\hline Dose Range [Gy] & df & $\begin{array}{c}\text { T-variable } \\
X^{2} \text { from the fit }\end{array}$ & $\begin{array}{c}\alpha_{\mathrm{sl}}=5 \% \\
\mathrm{X}^{2}(0.95, \mathrm{df})\end{array}$ & $\begin{array}{c}\text { Critical Region } \\
\mathrm{T}>\mathrm{X}^{2}(0.95, \mathrm{df})\end{array}$ & P-value \\
\hline $0-16$ & 31 & 124.58 & 44.99 & $\mathrm{H}_{0}:$ Rejected & $3.58 \times 10^{-13}$ \\
\hline $0-14$ & 27 & 38.20 & 40.11 & $\mathrm{H}_{0}:$ Not rejected & 0.07 \\
\hline $0-12$ & 23 & 25.78 & 35.17 & $\mathrm{H}_{0}:$ Not rejected & 0.31 \\
\hline $0-10$ & 19 & 23.85 & 30.14 & $\mathrm{H}_{0}:$ Not rejected & 0.20 \\
\hline $0-8$ & 15 & 18.37 & 25.00 & $\mathrm{H}_{0}:$ Not rejected & 0.24 \\
\hline $2-16$ & 27 & 109.05 & 40.11 & $\mathrm{H}_{0}:$ Rejected & $8.05 \times 10^{-12}$ \\
\hline $2-14$ & 23 & 24.89 & 35.17 & $\mathrm{H}_{0}:$ Not rejected & 0.36 \\
\hline $2-12$ & 19 & 11.98 & 30.14 & $\mathrm{H}_{0}:$ Not rejected & 0.89 \\
\hline $2-10$ & 15 & 10.63 & 25.00 & $\mathrm{H}_{0}:$ Not rejected & 0.78 \\
\hline $2-8$ & 11 & 6.77 & 19.68 & $\mathrm{H}_{0}:$ Not rejected & 0.82 \\
\hline $4-16$ & 23 & 106.19 & 35.17 & $\mathrm{H}_{0}:$ Rejected & $1.18 \times 10^{-12}$ \\
\hline $4-14$ & 19 & 23.89 & 30.14 & $\mathrm{H}_{0}:$ Not rejected & 0.20 \\
\hline $4-12$ & 15 & 10.67 & 25.00 & $\mathrm{H}_{0}:$ Not rejected & 0.78 \\
\hline $4-10$ & 11 & 9.57 & 19.68 & $\mathrm{H}_{0}:$ Not rejected & 0.57 \\
\hline $6-16$ & 19 & 86.72 & 30.14 & $\mathrm{H}_{0}:$ Rejected & $1.27 \times 10^{-10}$ \\
\hline $6-14$ & 15 & 17.44 & 25.00 & $\mathrm{H}_{0}:$ Not rejected & 0.29 \\
\hline $6-12$ & 11 & 2.34 & 19.68 & $\mathrm{H}_{0}:$ Not rejected & 0.997 \\
\hline $6-10$ & 7 & 1.64 & 14.07 & $\mathrm{H}_{0}:$ Not rejected & 0.977 \\
\hline
\end{tabular}

The table exhibits that almost in all the ranges, even including low dose data the LQ model cannot be rejected for a significance level of $5 \%$. However, like in Figs.4.6 and 4.7 for the $x^{2} /$ df statistics, an improvement of the LQ fit is seen by an increment of the P-values when low dose points are excluded. 


\subsubsection{Monte Carlo Simulation for CHOAA8 cell line}

In the last section, we presented the results of goodness of fit evaluation through numerical measures of $\mathrm{X}^{2}$ - test and P-value statistics for CHOAA8 cell line. In this section we present a graphical measure of the goodness of fit by way of plotting the T-variable and P-value distributions from one thousand similar experiments simulated using Monte Carlo technique. The results for $\mathrm{T}$ - variable and $\mathrm{P}$-Value in different ranges are shown in Figs. 4.8 and 4.9.

Monte Carlo analysis demonstrates dependence on dose range used in the data fitting. Fig.4.8 shows the T-distribution from $L Q$ fits displaying the " $\mathrm{X}^{2 \text { " variable in }}$ dark bars for CHOAA8 cell line. The theoretical $X^{2}$ is also plotted in solid lines. It is clear that the residuals are not $\mathrm{X}^{2}$ distributed for the total dose range from 0 to 16 Gy. Reducing the final dose of the range and keeping the initial dose equal to zero, the Monte Carlo distribution starts moving closer to the expected theoretical distribution. The best result is reached for the final dose equal to $13 \mathrm{~Gy}$; further reduction in the final dose does not noticeably improve the concordance between the actual and the expected distributions for the number of degrees of freedom involved. See Fig.4.8 in the ranges from 0 to $16,13,12$ and 10 Gy.

The next two cases displayed in Fig.4.8 are in the range from 2 to 13 Gy and from 5.5 to 13 Gy. There is a better agreement when the first 2 Gy range is excluded and the best concordance is reached for the fit between 5.5 and 13 Gy. Increasing the lower bound of the range does not substantially improve the 
agreement. In order to quantify the degree of correspondence between both distributions, the expectations of the theoretical $x^{2}$ - distribution, which is the number of degrees of freedom and the expectation value obtained from the Monte Carlo distribution, are shown in Table 4.5.

Table 4.5 Expectations for the theoretical and $M C X^{2}$ - distributions at several dose ranges. The difference is also displayed in the last column.

\begin{tabular}{|l|c|c|c|}
\hline Dose Range [Gy] & Expectation for $X_{\text {th }}^{2}$ & Expectation for $X^{2}$ MC & Difference \\
\hline $0-16$ & 31 & 155 & 124 \\
\hline $0-14$ & 27 & 65 & 38 \\
\hline $0-13$ & 25 & 51 & 26 \\
\hline $0-12$ & 23 & 49 & 26 \\
\hline $0-10$ & 19 & 43 & 24 \\
\hline $2-13$ & 21 & 34 & 13 \\
\hline $4-13$ & 17 & 28 & 11 \\
\hline $5.5-13$ & 14 & 17 & 3 \\
\hline $6-13$ & 13 & 16 & 3 \\
\hline
\end{tabular}

Figure 4.9 also shows that the best agreement between LQ model and the survival data is found in the region from 5.5 to $13 \mathrm{~Gy}$. Even though the complete flat distribution for the P-values is not achieved, the graphs exhibit an evident difference among several dose ranges. 


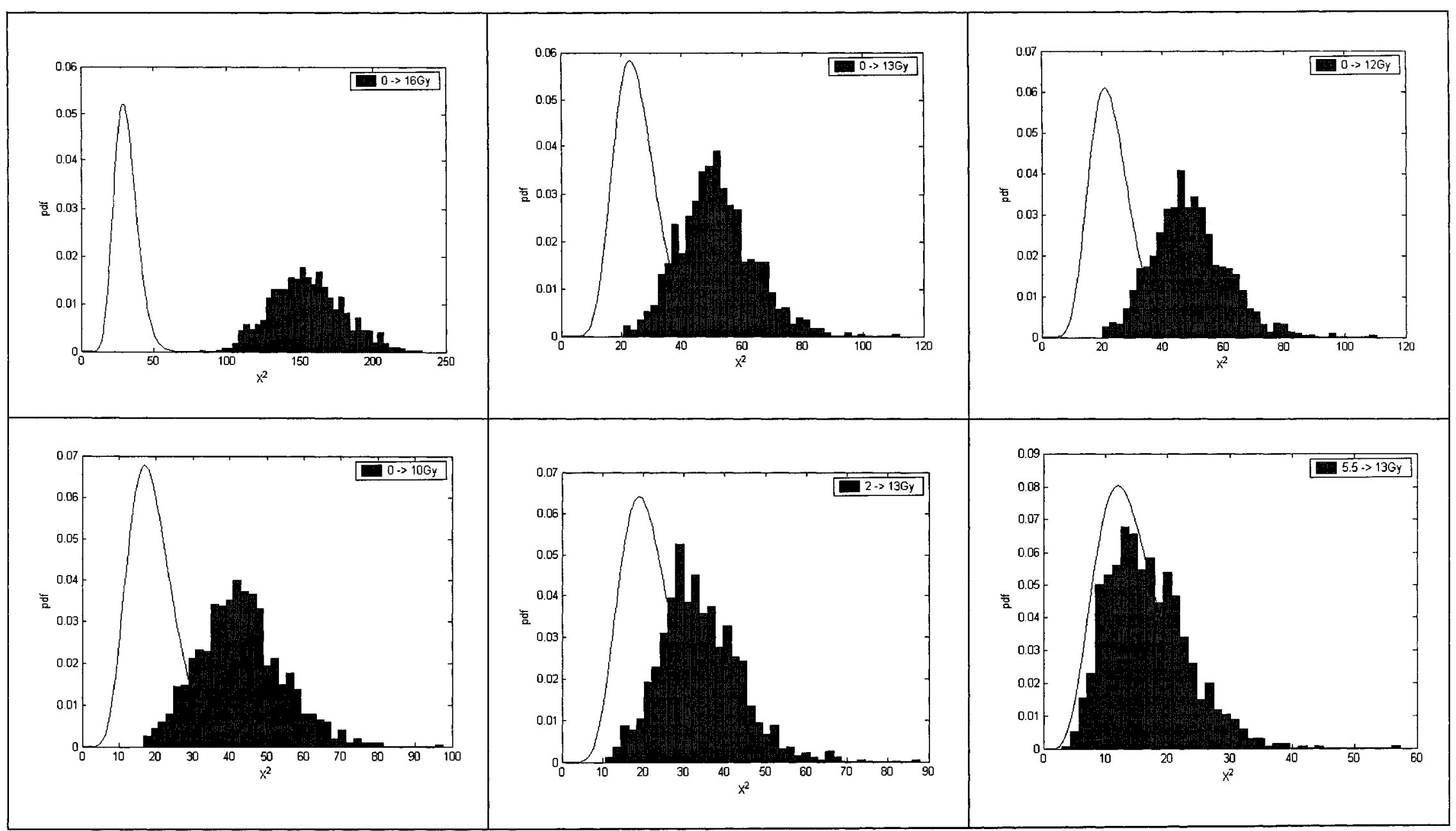

Fig. 4.8 Plots of Chi-Square distribution from Monte Carlo experiment for CHOAA8 in dark bars and the theoretical expected Chi-Square distribution in solid lines. The graphs show the distributions for different dose ranges (see the upper right corner of each graph). 


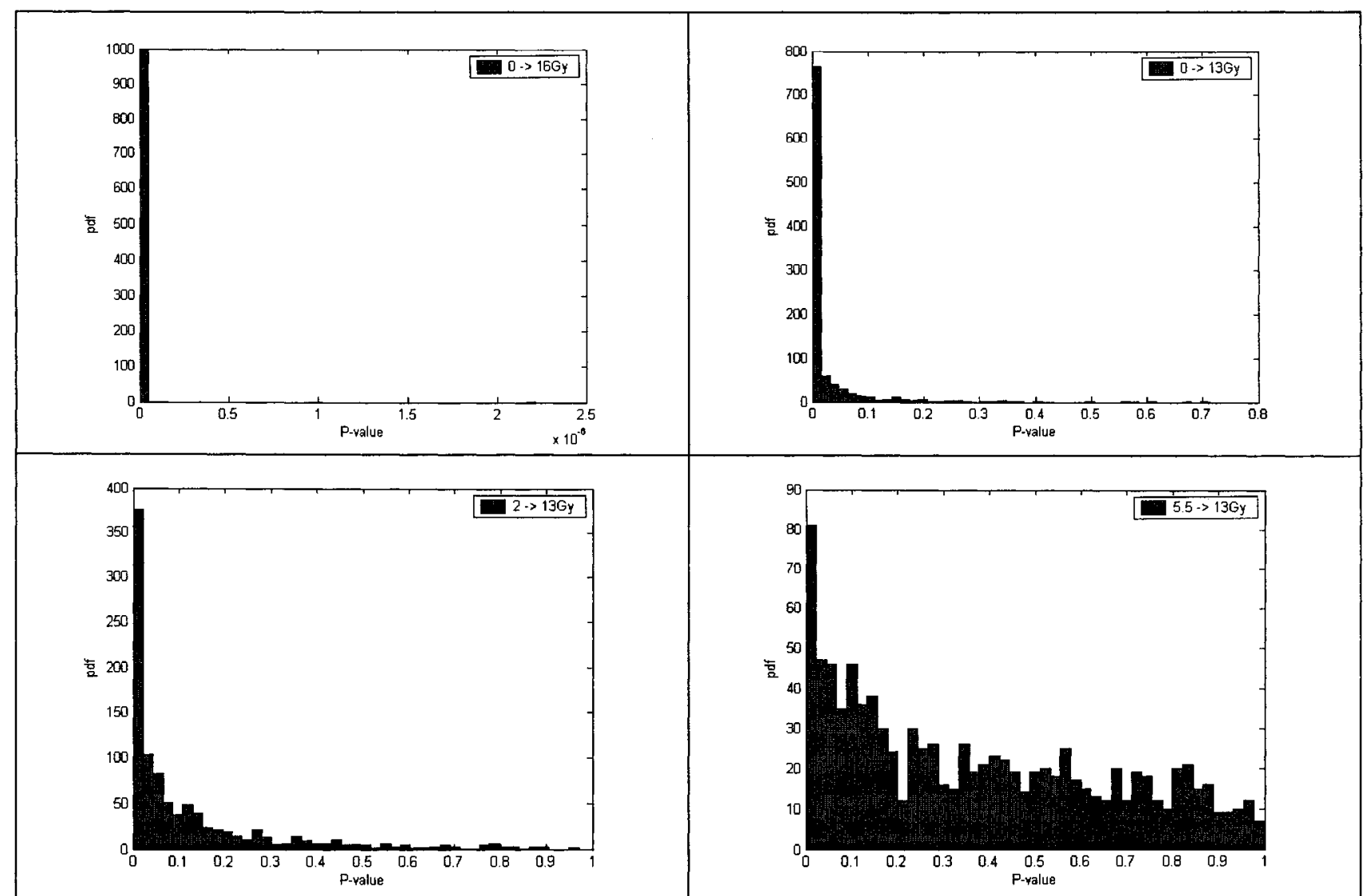

Fig. 4.9 P-value distribution from Monte Carlo experiment at different dose ranges for CHOAA8. One can see an evident difference among the different dose ranges although the complete flat distribution for P-value is not achieved. 


\subsubsection{Estimated parameter for CHOAA8 cell line.}

The least square estimators of the LQ fit to the survival data for several dose ranges are shown in Figs. 4.10 and 4.11. The parameters $\alpha$ and $\beta$ for LQ model were obtained as explained in section 3.2.2. The error bars represent the standard deviation of the fitted parameters, obtained by taking the square root of the diagonal elements from the covariance matrix.

Both parameters $\alpha$ and $\beta$ have big uncertainties at low dose ranges, especially for the $\beta$ parameter, which in general are smaller than $\alpha$ and have huge error bars in this region. However, this region is followed by a decrease of $\alpha$ and an increase of $\beta$ showing that the parameters $\alpha$ and $\beta$ from LQ model are negatively and highly correlated. The next region seems to have more stable values, with small changes when the high dose data are included. The figures also display no great difference between the parameters when the first 2.5 Gy are excluded especially if the final dose in the range reaches values $\geq 9 \mathrm{~Gy}$. Another feature is seen when the initial dose is fixed at 5.5 Gy. The parameters are very constant, no matter what is the final dose, unless it reaches values $>13$ Gy as shown before, and the curves undergo a small shift in that region. 


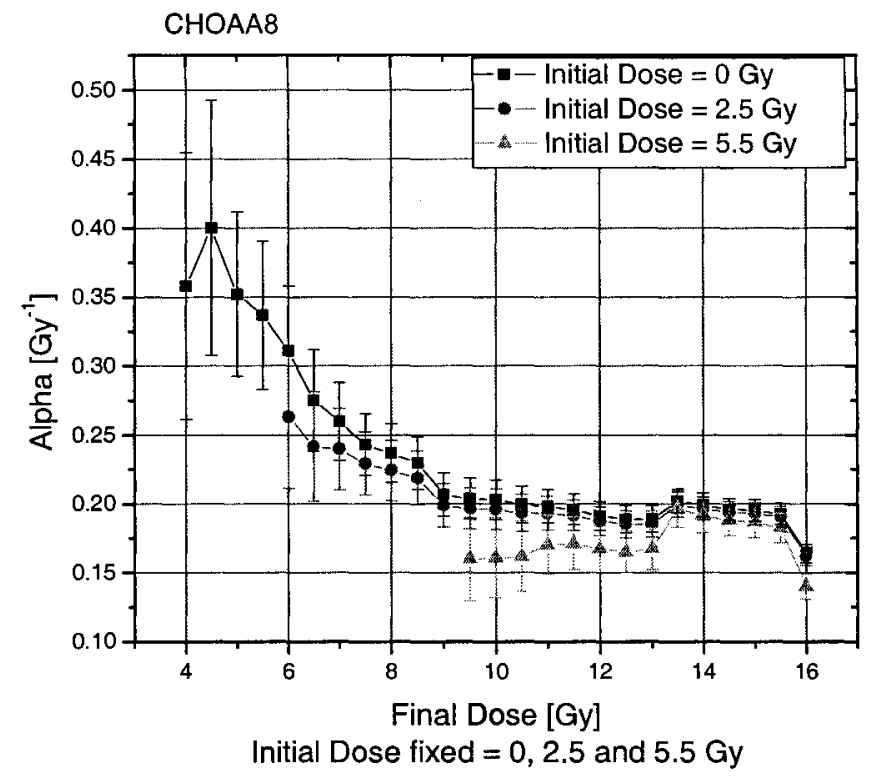

Fig.4.10 Alpha parameters for the LQ model obtained from LSM. Initial dose of the range is fixed at 0 , 2.5 and $5.5 \mathrm{~Gy}$.

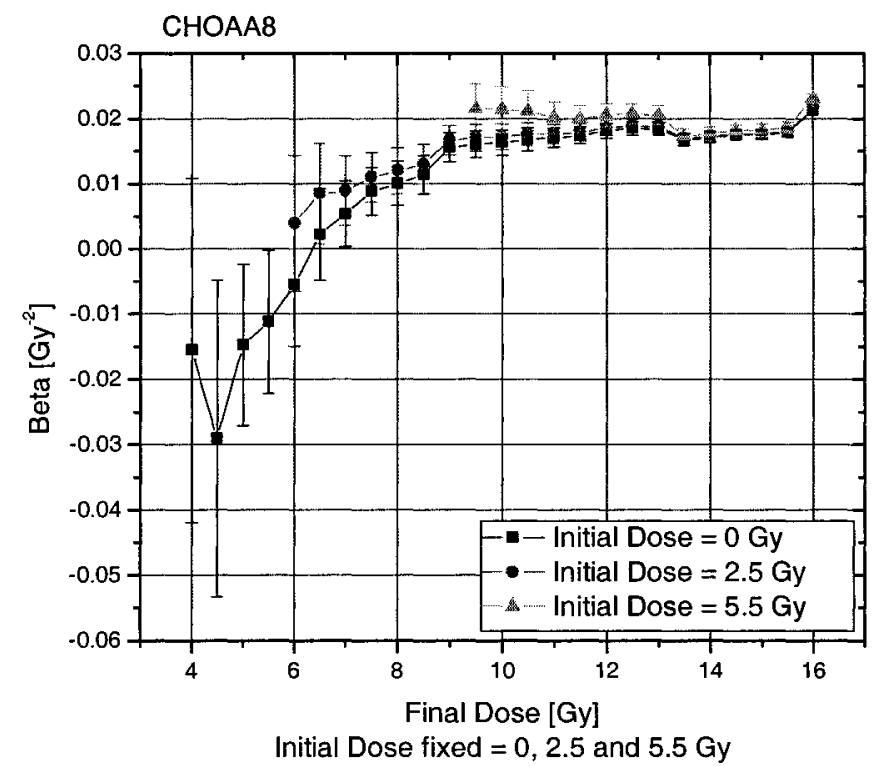

Fig.4.11 Beta parameters for the LQ model obtained from LSM. Initial dose of the range is fixed at 0 , 2.5 and 5.5 Gy. 
Table 4.6 summarizes some numerical values of $\alpha$ and $\beta$ parameters and $x^{2}$ statistics for several dose ranges presented before and Fig.4.12 graphically displays the result of the fit.

Table 4.6 The parameters $\alpha$ and $\beta$ from the LQ model least square fit to the CHOAA8 survival data, and their corresponding standard deviation $\sigma_{\alpha}$ and $\sigma_{\beta}$. The $x^{2} / d f$ statistics from the fit and the correlation coefficient between $\alpha$ and $\beta$ are also given.

\begin{tabular}{|l|c|c|c|c|c|c|}
\hline Dose Range $[\mathrm{Gy}]$ & $\begin{array}{c}\text { Alpha } \\
{\left[\mathrm{Gy}{ }^{-1}\right]}\end{array}$ & $\begin{array}{r}\sigma_{\alpha} \\
{\left[\mathrm{Gy}^{-1}\right]}\end{array}$ & $\begin{array}{r}\text { Beta } \\
{\left[\mathrm{Gy}^{-2}\right]}\end{array}$ & $\begin{array}{r}\sigma_{\beta} \\
{\left[\mathrm{Gy}^{-2}\right]}\end{array}$ & $\mathrm{X}^{2} \mathrm{df}$ & $\rho_{\alpha, \beta}$ \\
\hline $\begin{array}{l}\text { Total range } \\
0-16\end{array}$ & 0.164 & 0.007 & 0.0213 & 0.0006 & 4.02 & -0.94 \\
\hline $\begin{array}{l}\text { Excluding high doses } \\
0-13\end{array}$ & 0.189 & 0.010 & 0.0185 & 0.0010 & 1.06 & -0.96 \\
\hline $\begin{array}{l}\text { Excluding high and very low doses } \\
2.5-13\end{array}$ & 0.186 & 0.010 & 0.0188 & 0.0010 & 0.60 & -0.96 \\
\hline $\begin{array}{l}\text { Best LQ fit range found } \\
5.5-13\end{array}$ & 0.167 & 0.015 & 0.0205 & 0.0015 & 0.20 & -0.98 \\
\hline
\end{tabular}




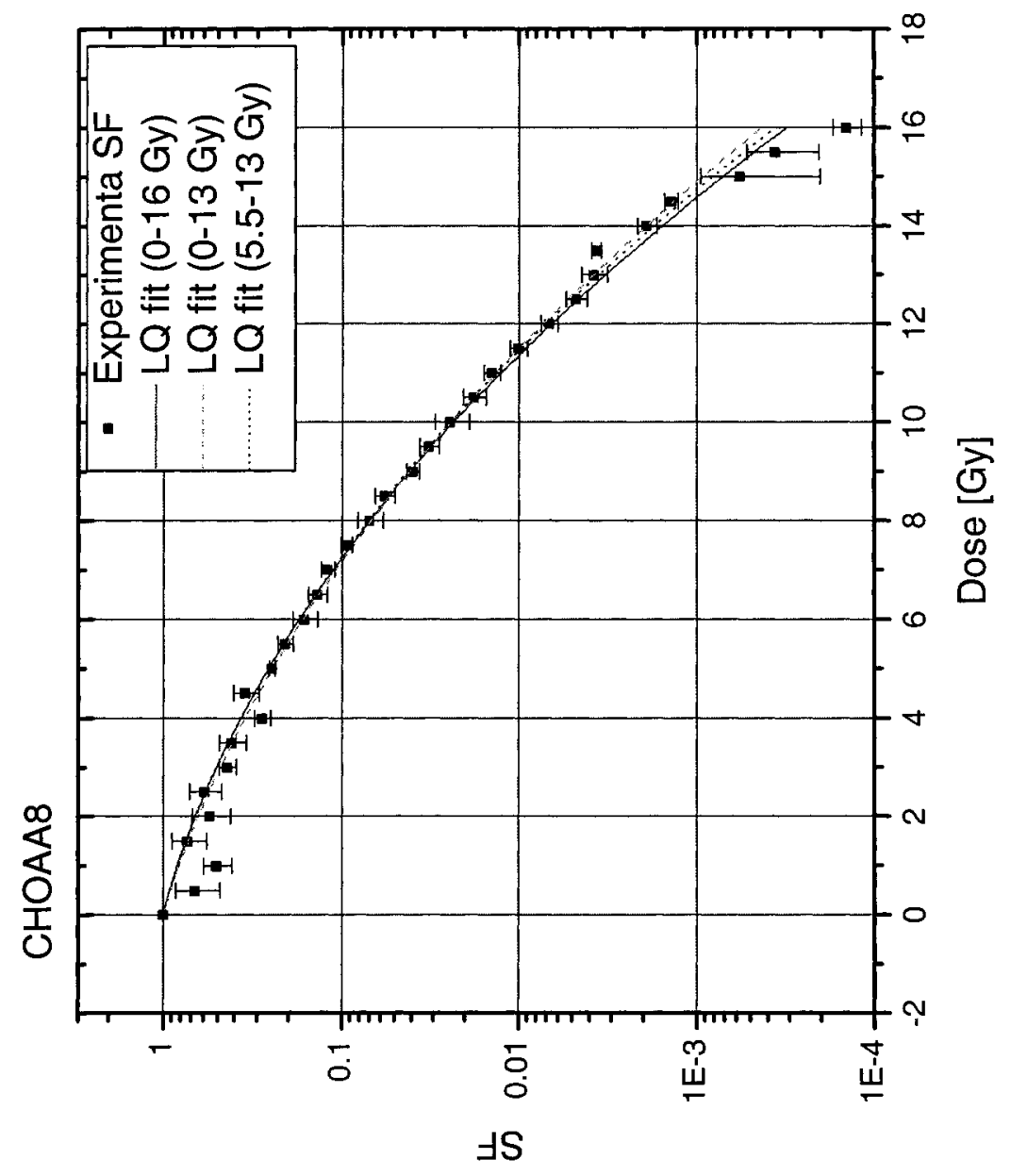






\subsubsection{Discussion}

The general shape of survival curve is described by an initial slope, followed by a shoulder and final linear trend for mammalian cells irradiated with $X$-rays, and the fact that the shape varies depending on cell line and dose range point out that the goodness of $L Q$ relationship to fit the data will also depend on cell line and dose range.

The least squares fitting method gives a powerful tool for evaluating the goodness of fit. The $x^{2} / d f$ statistics and hypothesis test, together with Monte Carlo simulation and a residuals exam, are used to carry out the $L Q$ dose range analysis.

In the section 3.2.1 we described how $x^{2} / \mathrm{df}$ is a measure of the agreement between the observed data and the hypothesis and the conditions assumed by the data. The functional form for the hypothesis is in this case the LQ model. Figures 4.6 and 4.7 clearly show several levels in $x^{2} /$ df variability for CHOAA8 cell line; therefore the discussion will be centered in some regions: very low dose region ( $<1 \mathrm{~Gy}$ ), low dose region defined by the first few Gray, middle dose region, and high dose region.

A decline of fit quality at very low doses ( $<1 \mathrm{~Gy}$ ) based on $x^{2} / \mathrm{df}$ is observed in the cell line. This result is explained by the increase of radiosensitivity observed in CHOAA8. An HRS/RR- type response has not been reported before for this 
cell line. Therefore, to ensure its existence, maybe a more detailed analysis must be done. However, it is obvious that $L Q$ formalism could only explain the general trend of the survival curve.

The LQ model cannot describe the hypersensitive effect because the mechanistic basis on which it is founded is not able to explain the response to very low-dose radiation, especially when cells respond uniquely to very low-dose radiation, i.e. the mechanisms of response for low and high doses are different.

In addition, a very clear improvement of the fit is discerned at slightly higher doses. Certainly, removing the first $4 \mathrm{~Gy}$ from the fit causes $\mathrm{X}^{2} / \mathrm{df}$ to drop $81 \%$. This could be a result of the linearity existent in the trend of survival curve at low doses that will affect the total fit in a range from 0 Gy to final dose. However, there are other factors that could influence the improvement in the fit. One could be a possible decrease of uncertainties within this dose range, and the other one is that the surviving fraction at dose point of 4 Gy seems to be lower than the general trend of the curve, indicating that it might be an outlier.

The study of the experimental uncertainties along the survival curve exhibited that there is an increase of the error bars at low doses, with relative errors bigger than $20 \%$ but just within the first 2.5 Gy range. Hence, the big step down in the $\mathrm{X}^{2} / \mathrm{df}$ plot at $4.5 \mathrm{~Gy}$ in Fig.4.7 might be not related to the uncertainties. Although we will come back later to the influence of the uncertainties in the LQ fit, we initially explored the other possibility. 
Hence, we repeated the same procedure as in Fig. 4.7 but removing the point at dose of 4 Gy. The analysis revealed a shift down in the first 4 Gy of the surviving fraction. See Table 4.7 and compare with the value in Fig. 4.7. Moreover, a fluctuating but dropping of $X^{2} / \mathrm{df}$ quantity between 1.5 and 5.5 Gy is observed as shown in Fig. 4.13. Above 5.5 Gy the $x^{2} / d f$ quantity seems to stabilize approximately around 0.199 .

Table 4.7 Chi-Square divided by the number of degrees of freedom at different dose ranges and excluding 4Gy data point in the first two columns and excluding 13.5Gy in the last two columns for CHOAA8. The points are excluded due to the fact that they may be outliers.

\begin{tabular}{|l|l|l|c|}
\hline \multicolumn{2}{|c|}{4 Gy excluded } & \multicolumn{2}{c|}{13.5 Gy excluded } \\
\hline Dose Range [Gy] & $X^{2} / \mathrm{df}$ & Dose Range [Gy] & $\mathrm{X}^{2} / \mathrm{df}$ \\
\hline $0-13$ & 0.8475 & $5.5-12$ & 0.2035 \\
\hline $0.5-13$ & 0.8843 & $5.5-12.5$ & 0.1960 \\
\hline $1-13$ & 0.8386 & $5.5-13$ & 0.1960 \\
\hline $1.5-13$ & 0.2836 & $5.5-13.5$ & 0.1960 \\
\hline $2-13$ & 0.2978 & $5.5-14$ & 0.2097 \\
\hline $2.5-13$ & 0.2867 & $5.5-14.5$ & 0.2521 \\
\hline $3-13$ & 0.3006 & $5.5-15$ & 0.2805 \\
\hline $3.5-13$ & 0.2473 & $5.5-15.5$ & 0.3829 \\
\hline $4-13$ & 0.2614 & & \\
\hline $4.5-13$ & 0.2614 & & \\
\hline $5-13$ & 0.2351 & & \\
\hline $5.5-13$ & 0.1961 & & \\
\hline $6-13$ & 0.2040 & & \\
\hline $6.5-13$ & 0.1982 & & \\
\hline $7-13$ & 0.2009 & & \\
\hline $7.5-13$ & 0.1975 & & \\
\hline
\end{tabular}




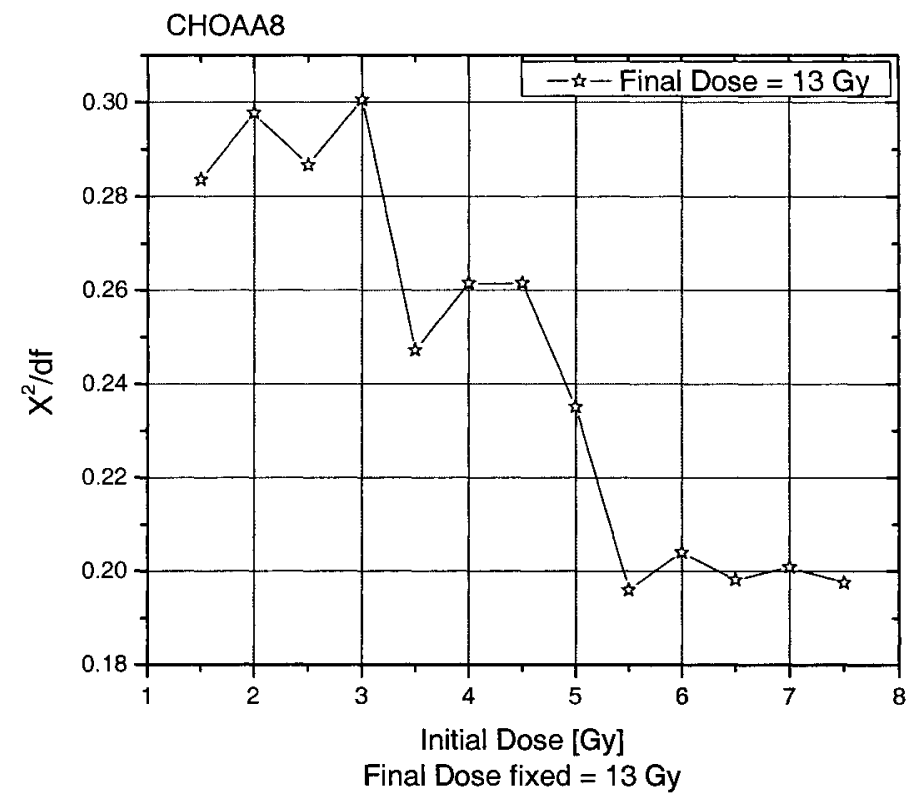

Fig. 4.13 Chi-Square divided by the number of degrees of freedom at different dose ranges and excluding $4 \mathrm{~Gy}$ data point for CHOAA8. Final dose of the range is fixed at $13 \mathrm{~Gy}$. Initial dose of the range varies along the $X$-axis.

Therefore, it seems that LQ model works better in the middle dose region within the range from 5.5 to 13 Gy. This is confirmed by examination of the residuals in the region for different dose range fits. The hypothesis of linearity for low dose region might be consistent since the $L Q$ relationship overestimates the experimental survival curve in the first 4 Gy region with residuals systematically negative for the fits at different dose ranges. The residuals between the $L Q$ model and the data as in Eq.3.8 are shown in Fig.4.14. 


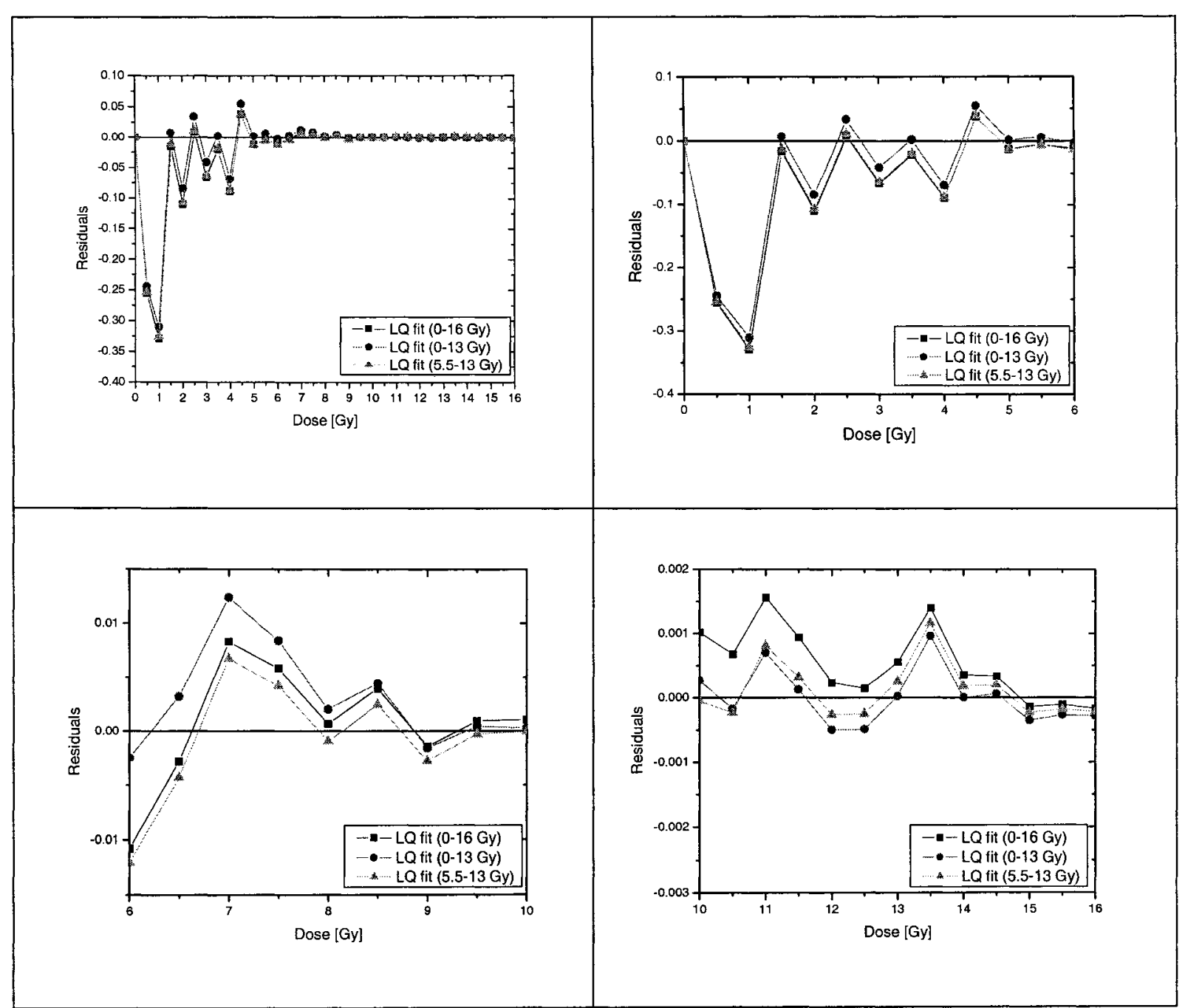

Fig.4.14. Residuals from LQ fit for CHOAA8. The plots show the total range investigated (upper-left corner) and a more detailed behavior of the same residuals for smaller dose scales: (0-6) Gy in upper-right corner, (6-10) Gy in lower-left corner, (10-16) Gy in lower-right corner. 
Figures 4.12 and 4.14 reflect that $L Q$ model does not properly fit the data at low doses but also at high dose region. The analysis of $x^{2} / d f$ and residuals exhibit a threshold at $13 \mathrm{~Gy}$, but at dose equal 13.5 Gy the data point has a 'suspicious' higher value in this case. Then, the procedure was repeated fitting from 5.5Gy until different final dose at high dose region excluding 13.5 Gy data point. The initial dose in this case was fixed at 5.5 Gy to remove the bad influence of low dose region. The results are shown in the last two columns of Table 4.7. A threshold is still seen at 14 Gy.

On the other hand, a $x^{2}$-test for goodness of fit for a significance level of $5 \%$ displayed that the hypothesis of LQ relationship even including the hypersensitive and low dose regions cannot be rejected in almost all the dose ranges from 0 to 15 Gy. The only region where the hypothesis can be safely rejected is when including $16 \mathrm{~Gy}$. See Table 4.4. The large errors coming from the clonogenic survival technique applied to the CHOAA8 cell line ensure small values of $X^{2}$ and consequently large $P$-values from the $L Q$ fit for the most of the dose ranges. In fact, some fits are better than expected given the size of the measurement errors yielding values of $x^{2} / d f$ much less than one. That is why, if the data could be considered independent and Gaussian distributed with known errors and the hypothesis were true, by the data samples obtained in this experiment, the LQ model would not be refuted in any range from 0 to 15 Gy. 
In spite of big uncertainties we still want to do relative comparison and define regions of better agreement and certainly which we already did by calculating $\mathrm{X}^{2} / \mathrm{df}$ and comparing them as shown in Figs. 4.6 and 4.7. Looking to Eq.3.14 or 3.16 it is seen that $T$ is a measure of the agreement between the hypothesis and measured data since quantity $u_{i}$ in Eq.3.13 is a measure of the deviation between the $\mathrm{i}^{\text {th }}$ measurement and the value predicted by the hypothesis. Even though the data do not meet the conditions explained in the previous paragraph, Eqs.3.14 and 3.16 still give a measure of the deviation between the model and the data but no statistical inference could be done, for which we need the conditions in Eqs.3.11 and 3.12 to be satisfied. That is why verifying the residuals is another way to evaluate the goodness of fit as it seen in Fig. 4.14.

One of the reasons of not having standard normally distributed errors is that the model is not the right choice for the data, and then the T variable in Eq.3.14 will not be $x^{2}$ distributed either. Therefore, thousand of generated Monte Carlo data similar to the experiment will show the distribution of $\mathrm{T}$ variable. Note that as we explained in section 3.2.4 we are not using here Monte Carlo in the regular way of improving statistical accuracy of estimated parameter by increasing the data sample. We are assuming that the average from in total 9 experiments at each data point is giving the value of surviving fraction closest to the actual value. We know that the arithmetic mean of a random variable is a random variable itself; then, this is an assumption, the same way we assume that the errors are known 
and equal to the standard error of the mean from the experiment. However, this cannot be used to quantify statistical accuracy of the parameters since we cannot account for the bias introduced when assuming that the mean of experimental surviving fraction is the actual value; it is just used to see relative discrepancy between LQ model and the data at different dose ranges. Figures 4.8 and 4.9 for the $\mathrm{T}$ and $\mathrm{P}$-value distributions show that the results are in correspondence with the $\mathrm{X}^{2} / \mathrm{df}$ outcome, indicating that the LQ model seems to fit the data better in the dose range from 5.5 to $13 \mathrm{~Gy}$.

Finally, Figs. 4.10 and 4.11 display that the fitted parameters $\alpha$ and $\beta$ are not known accurately for the low dose range. The small quadratic component and its huge error bars indicate that this term is not contributing to improve the LQ fit. This region is followed by a portion of high variability in the coefficients due to their correlation. At last, the constancy in the parameters supports the theory of LQ behaviour at middle doses since when a major part of this region is included in the fit, adding more points to the fit will not change the estimated value.

Starting from the general survival shape, described as initial linear followed by a linear quadratic response at middle doses and final slope at higher doses, we could predict that there are three main regions involved and influencing the fit for CHOAA8 cell line. First, we have a linear region (L Region) with larger values of alpha than beta parameters. However, we are not discussing this region in detail because it is not the objective of this study and the region is substantially 
affected by the HRS/RR effect, but at least it could be said that LQ coefficients are not accurately determined. The boundary between the linear and the following linear quadratic region is characterized by strong $\alpha$ and $\beta$ variability. The linear quadratic region (LQ Region) is characterized by an improvement of goodness of fit and Monte Carlo experiment outcome. Finally, there is a high dose region that $L Q$ model cannot explain. 


\subsubsection{Results of data analysis for CP3 cell line}

\subsubsection{Evaluating goodness of fit for $\mathrm{CP} 3$ cell line}

Figure 4.15 shows $X^{2} / d f$ from the $L Q$ fit at different dose ranges. The results are now plotted for CP3 cell line for fixing the initial dose at 0, 2.5 and 5.5 Gy while the final dose varies along the $\mathrm{X}$-axis of the graph as we did for CHOAA8.

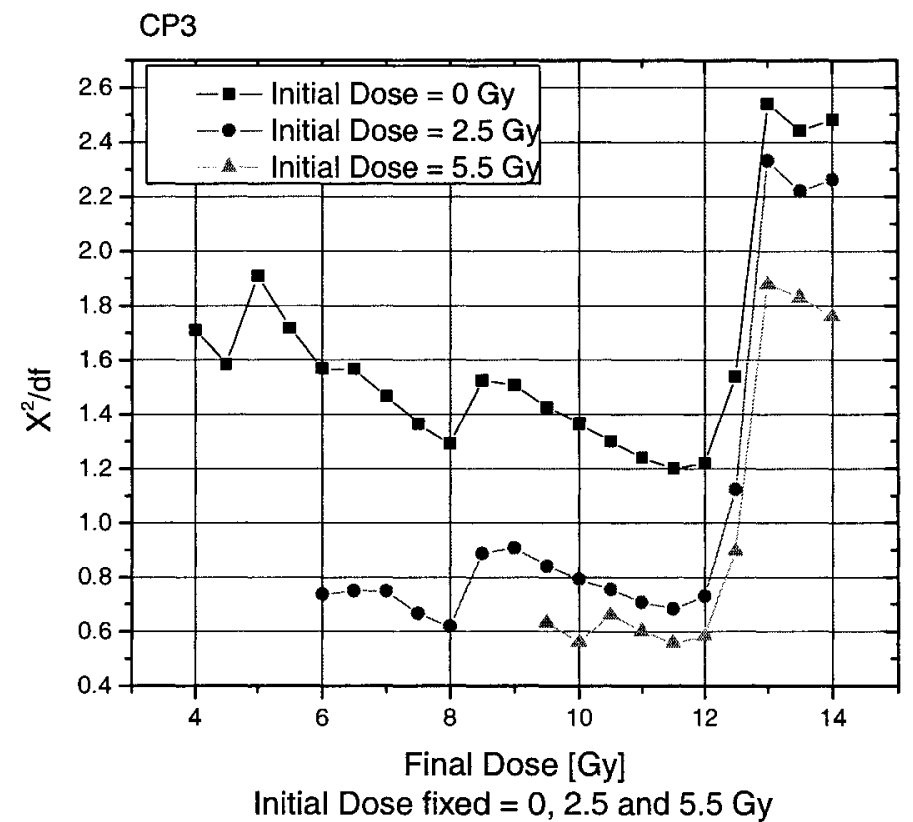

Fig. 4.15 Chi-Square divided by the number of degrees of freedom at different dose ranges for CP3 cell line. Initial dose of the range is fixed at $0,2.5$ and $5.5 \mathrm{~Gy}$. Final dose of the range varies along the $\mathrm{X}$-axis.

The figures show a variability of $x^{2} / d f$ depending on dose range used in the fit.

From Fig. 4.15 we can see that at some point for high doses the fit worsens 
defining a clear threshold around 12 Gy where $x^{2} / \mathrm{df}$ starts increasing. There are two shifts observed when the data points below 2.5 Gy and 5.5 Gy are excluded. However, it is obvious that the biggest improvement is seen when the first 2.5 Gy data are removed, which is confirmed from the analysis fixing the final dose while changing the initial dose as seen in Fig. 4.16.

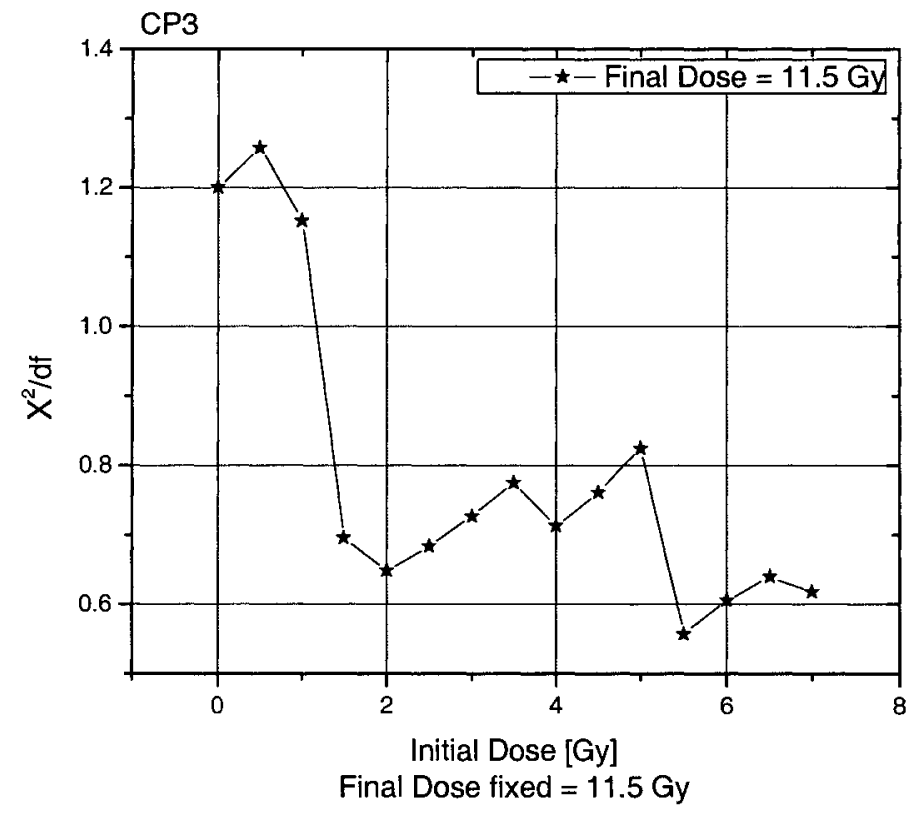

Fig. 4.16 Chi-Square divided by the number of degrees of freedom at different dose ranges for CP3. Final dose of the range is fixed at $11.5 \mathrm{~Gy}$. Initial dose of the range varies along the $\mathrm{X}$-axis.

The figure 4.16 shows a level with mean value of the $x^{2} /$ df statistics around 1.20 at very low doses ( $\leq 1 \mathrm{~Gy}$ ). Some fluctuations around $x^{2} / d f \approx 0.73$ are following between 1.5 and $5 \mathrm{~Gy}$, and a next step from $5.5 \mathrm{~Gy}$ around $x^{2} / \mathrm{df} \approx 0.61$. The 
analysis reflects a fit improvement of $39 \%$ just excluding the range $\leq 1 \mathrm{~Gy}$. If the low dose data up to 5 Gy are not included $x^{2} /$ df drops in total by $49 \%$.

The results of testing the LQ hypothesis for a significance level of $5 \%$ for several dose ranges are presented in Table 4.8.

Table $4.8 x^{2}$-Test for CP3 cell line at several dose ranges. Testing the LQ hypothesis for a significance level of $5 \%$. In the last column the P-value is also shown.

\begin{tabular}{|c|c|c|c|c|c|}
\hline $\begin{array}{l}\text { Dose Range } \\
\text { Gy] }\end{array}$ & df & $\begin{array}{l}\text { T-variable } \\
\mathrm{X}^{2} \text { from the fit }\end{array}$ & $\begin{array}{c}\alpha_{s l}=5 \% \\
x^{2}(0.95, d f)\end{array}$ & $\begin{array}{l}\text { Critical Region } \\
T>x^{2}(0.95, d f)\end{array}$ & P-value \\
\hline $0-14$ & 27 & 67.00 & 40.11 & $\mathrm{H}_{0}:$ Rejected & $2.97 \times 10^{-05}$ \\
\hline $0-12$ & 23 & 28.08 & 35.17 & $\mathrm{H}_{0}:$ Not rejected & 0.21 \\
\hline $0-10$ & 19 & 25.95 & 30.14 & $\mathrm{H}_{0}:$ Not rejected & 0.13 \\
\hline $0-8$ & 15 & 19.39 & 25.00 & $\mathrm{H}_{0}:$ Not rejected & 0.20 \\
\hline $2-14$ & 23 & 49.83 & 35.17 & $\mathrm{H}_{0}:$ Rejected & $9.71 \times 10^{-04}$ \\
\hline $2-12$ & 19 & 13.18 & 30.14 & $\mathrm{H}_{0}:$ Not rejected & 0.83 \\
\hline $2-10$ & 15 & 11.13 & 25.00 & $\mathrm{H}_{0}:$ Not rejected & 0.74 \\
\hline $4-14$ & 19 & 45.87 & 30.14 & $\mathrm{H}_{0}:$ Rejected & $5.15 \times 10^{-04}$ \\
\hline $4-12$ & 15 & 11.39 & 25.00 & $\mathrm{H}_{0}:$ Not rejected & 0.7246 \\
\hline $4-10$ & 11 & 9.35 & 19.68 & $\mathrm{H}_{0}:$ Not rejected & 0.59 \\
\hline
\end{tabular}

The table shows that the LQ model for a significance level of $5 \%$ cannot be rejected except when the highest dose point is included, for which the hypothesis can be safely rejected, not just $T>X^{2}(0.95, d f)$ but the $P$-value is also very small. However, like in Figs. 4.15 and 4.16 for the $x^{2} /$ df statistics, an improvement of the LQ fit is seen by an increment of the P-values when low dose points are excluded. 


\subsubsection{Monte Carlo Simulation for CP3 cell line}

Monte Carlo analysis for CP3 cell line exhibits dependence on dose range used in the data fitting as well. Figure 4.17 shows the T-distribution from the LQ fits, which are in dark bars and the theoretical $x^{2}$ is also plotted in solid lines as before. It is clear that the residuals are not $x^{2}$ distributed for the total dose range from 0 to 14 Gy. Reducing the final dose of the range and keeping the initial dose at zero, the Monte Carlo distribution starts moving closer to the expected theoretical distribution. The best result is reached for the final dose equal to 11.5 Gy; further reduction in the final dose will not noticeably improve the concordance between the actual and the expected distributions for the number of degrees of freedom involved. See the graph in the ranges from 0 to $14,11.5$ and 10 Gy.

The next displayed cases in Fig.4.17 are in the range from 2, 4 and 5.5 Gy to $11.5 \mathrm{~Gy}$. There is a better agreement when the first 2 Gy range is excluded and the best concordance is reached for the fit between 5.5 and 11.5 Gy. Increasing the lower bound of the range does not improve the agreement. The expectations of the theoretical $\mathrm{X}^{2}$-distribution, which is the number of degrees of freedom and the expectation value obtained from the Monte Carlo distribution, are shown in Table 4.9 as we did for CHOAA8 cell line. 
Table 4.9 Expectations for the theoretical and $M C \mathrm{x}^{2}$ - distributions at several dose ranges for CP3 cell line. The difference is also displayed in the last column.

\begin{tabular}{|l|c|c|c|}
\hline Dose Range [Gy] & Expectation for $X^{2}$ in & Expectation for $X^{2}{ }_{\text {MC }}$ & Difference \\
\hline $0-14$ & 27 & 94 & 67 \\
\hline $0-12$ & 23 & 51 & 28 \\
\hline $0-11.5$ & 22 & 49 & 27 \\
\hline $0-10$ & 19 & 45 & 26 \\
\hline $0-8$ & 15 & 35 & 20 \\
\hline $2-11.5$ & 18 & 30 & 12 \\
\hline $4-11.5$ & 14 & 24 & 10 \\
\hline $5.5-11.5$ & 11 & 17 & 6 \\
\hline $6-11.5$ & 10 & 16 & 6 \\
\hline
\end{tabular}

Figure 4.18 displays the P-value distribution, which is not flat as well, but it also reveals that the best agreement between the LQ model and the survival data is found in the region from 5.5 to 11.5 Gy. 




Fig. 4.17 Plots of Chi-Square distribution from Monte Carlo experiment for CP3 in dark bars and the theoretical expected Chi-Square distribution in solid lines. The graphs show the distributions for different dose ranges (see the upper right corner of each graph). 


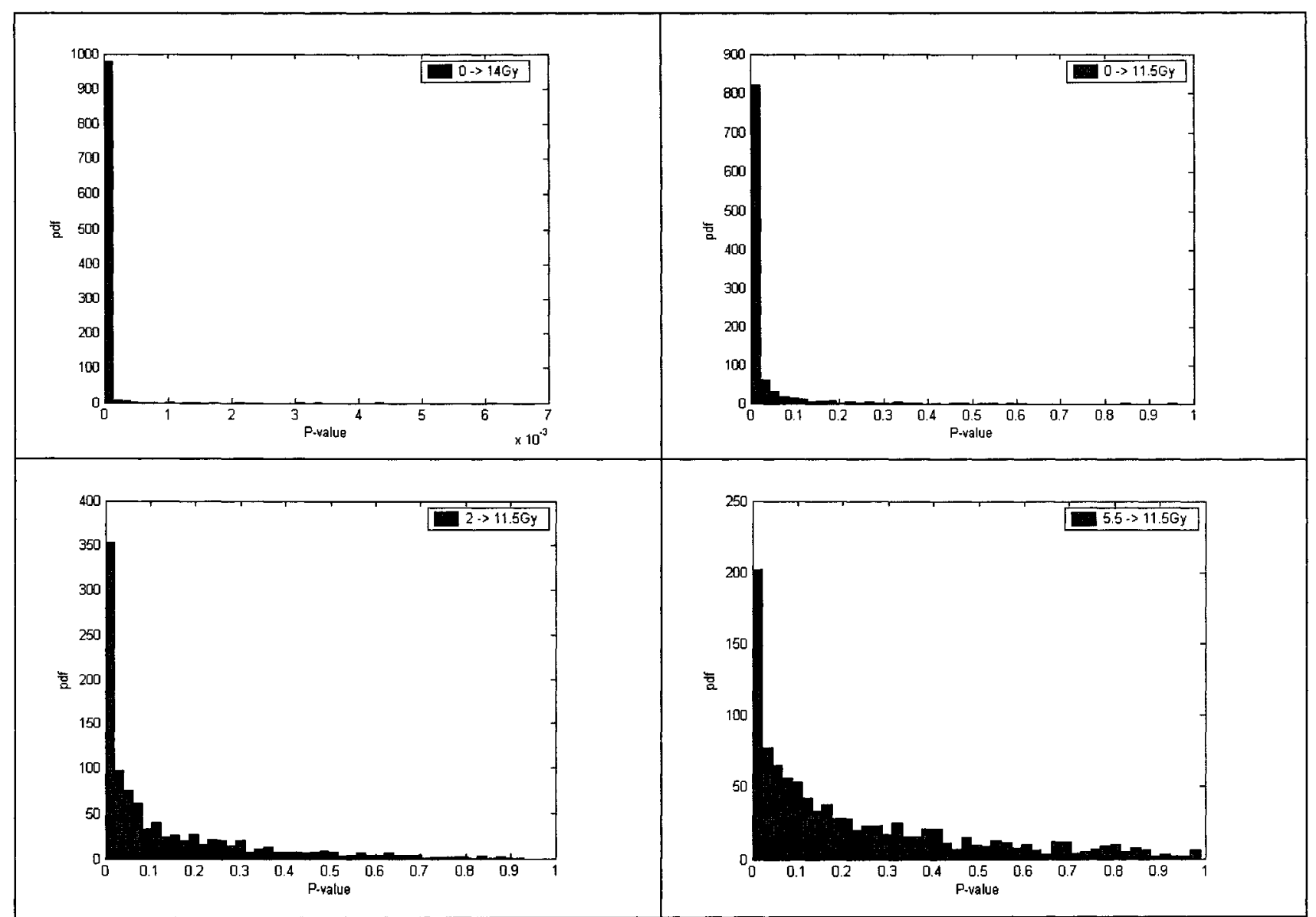

Fig. 4.18 P-value distribution from Monte Carlo experiment at different dose ranges for CP3. One can see an evident difference among the different dose ranges although the complete flat distribution for P-value is not achieved. 


\subsubsection{Estimated parameter for $\mathrm{CP} 3$ cell line.}

The least square estimators for the parameters of the $L Q$ fit to the survival data for several dose ranges are shown in Figs. 4.19 and 4.20.

The LQ parameters $\alpha$ and $\beta$ have big uncertainties at low dose ranges, but for CP3 cell line it becomes very large for $\alpha$ parameters, which in general are smaller than $\beta$ and have huge error bars in this region. This region is followed by an increase of $\alpha$ and a decrease of $\beta$ showing their inverse correlation. The values at last stabilize between 8.5 and 12 Gy. There is another small step when the high dose data is included. The figures also display no great difference between the parameters when the first 2.5 Gy are excluded, similar to the $\mathrm{CHO}$ cell line, especially if the final dose in the range reaches values $\geq 8.5$ Gy. Even though the first 2.5 Gy of the survival is responsible for the $x^{2} /$ df-statistics increase, it seems to have no great influence in the estimated coefficients at middle doses. Shift up for $\alpha$ and down for $\beta$ parameters is also seen when the initial dose of the range is fixed at $5.5 \mathrm{~Gy}$. 


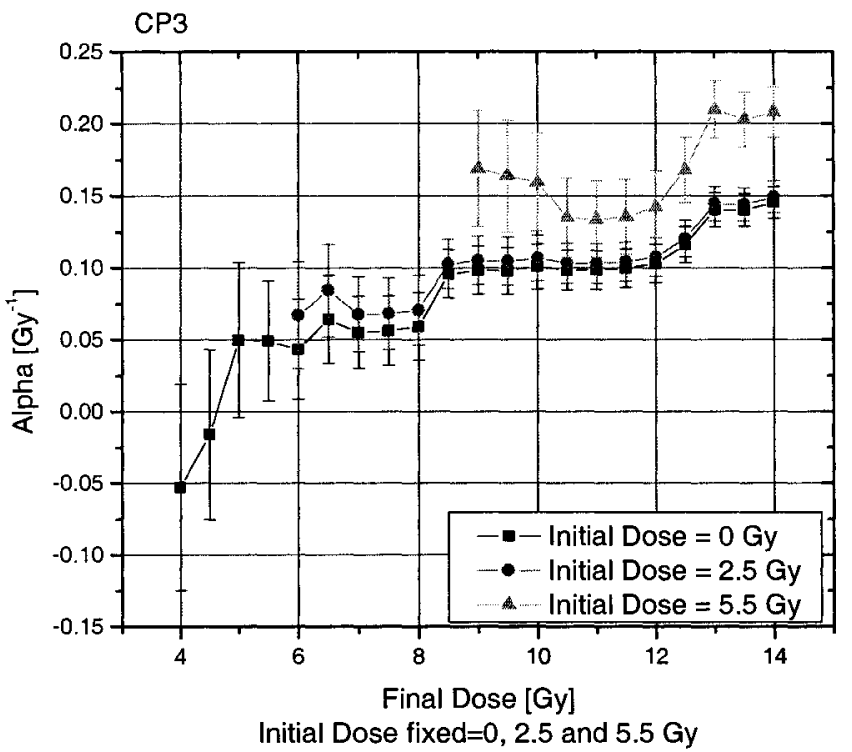

Fig.4.19 Alpha parameters for the LQ model obtained from LSM. Initial dose of the range is fixed at 0 , 2.5 and 5.5 Gy. CP3 cell line.



Fig.4.20 Beta parameters for the LQ model obtained from LSM. Initial dose of the range is fixed at 0 , 2.5 and 5.5 Gy. CP3 cell line. 
Table 4.10 summarizes some numerical values of $\alpha$ and $\beta$ parameters and $x^{2}-$ statistics for several dose ranges presented before. In Fig. 4.21 the data and the LQ fits are plotted.

Table 4.10 The parameters $\alpha$ and $\beta$ from LQ model least square fit to the CP3 survival data. Their corresponding standard deviation $\sigma_{\alpha}$ and $\sigma_{\beta}$. The $\chi^{2} / \mathrm{df}$ statistics from the fit and the correlation coefficient between $\alpha$ and $\beta$.

\begin{tabular}{|l|c|c|c|c|c|c|}
\hline Dose Range [ Gy] & $\begin{array}{c}\text { Alpha } \\
{\left[\mathrm{Gy}^{-1}\right]}\end{array}$ & $\begin{array}{r}\sigma_{\alpha} \\
{\left[\mathrm{Gy}^{-1}\right]}\end{array}$ & $\begin{array}{c}\text { Beta } \\
{\left[\mathrm{Gy}^{2}\right]}\end{array}$ & $\begin{array}{c}\sigma_{\beta} \\
{\left[\mathrm{Gy}^{-2}\right]}\end{array}$ & $\mathrm{x}^{2} / \mathrm{df}$ & $\rho_{\alpha, \beta}$ \\
\hline $\begin{array}{l}\text { Total range } \\
0-14\end{array}$ & 0.146 & 0.011 & 0.0443 & 0.0013 & 2.48 & -0.94 \\
\hline $\begin{array}{l}\text { Excluding high doses } \\
0-11.5\end{array}$ & 0.100 & 0.014 & 0.0518 & 0.0020 & 1.20 & -0.95 \\
\hline $\begin{array}{l}\text { Excluding high and very low doses } \\
2.5-11.5\end{array}$ & 0.104 & 0.014 & 0.0512 & 0.0020 & 0.68 & -0.95 \\
\hline $\begin{array}{l}\text { Best LQ fit range found } \\
5.5-11.5\end{array}$ & 0.136 & 0.026 & 0.048 & 0.003 & 0.56 & -0.98 \\
\hline
\end{tabular}




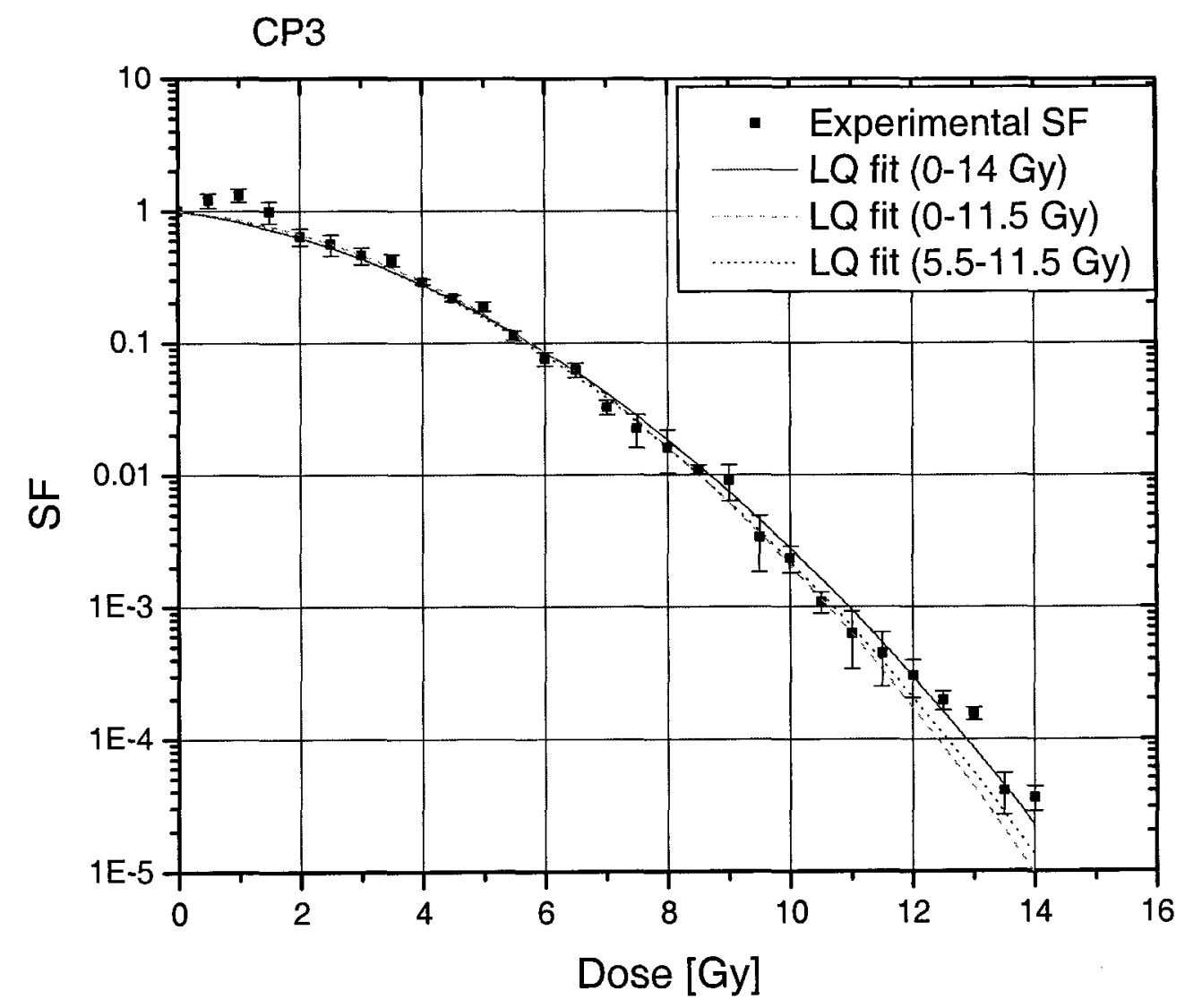

Fig. 4.21 LQ fit at different dose ranges for CP3 cell line. The LQ models are extrapolated to the total dose range. 


\subsubsection{Discussion}

A decline of fit quality at very low doses ( $\leq 1 \mathrm{~Gy}$ ) based on $x^{2} / \mathrm{df}$ is observed in the CP3 cell line. The result is explained by what appears to be an adaptive response. This increase of the survival fraction is related to the growing ability of the cells to adapt to the new environment, triggered by a small dose of radiation. The clonogenic survival assay normalizes the surviving fraction to the number of cells that survive after being plated when no radiation is given, that way the contribution of dying cells not due to radiation damage is removed. Thus, what the curve is showing is that the cells under very low-dose radiation develop a defence mechanism, which makes them able to adapt to the new conditions. The increase in the survival curve at very low doses causes high uncertainty in the estimated parameters when the low dose data are included in the fit.

Even though $x^{2} / d f$ statistic shows a bigger impact due to the influence of the first 1 Gy segment of the survival curve data (see Figs.4.15 and 4.16), the Monte Carlo outcome in Table 4.9 exhibits great effects of eliminating the initial 5.5 Gy from the fit. See also Figs. 4.17 and 4.18. For the CP3 cell line the agreement between the theoretical and the actual expectation of T-distribution is affected by the increase of the experimental uncertainties at middle doses. Moreover, it is this range from 0 to 5.5 Gy that results in a noticeable change of LQ estimators. 
The residuals between $L Q$ model and the experimental survival curve also reflect that the model systematically underestimate the surviving fraction in the low dose region. See Fig.4.22. Moreover, the residuals appear to behave randomly for the fit in the region from 5.5 to $11.5 \mathrm{~Gy}$.

Therefore, the $L Q$ formalism seems to work better in the middle region between 5.5 and 11.5 Gy for the data set presented here. In order to be more precise it is necessary to reduce the uncertainties especially in the middle and high dose region. Trying to establish and define a possible linear region will need a more careful analysis since it is evident that the adaptive response has a huge impact in the fitted coefficients and it is present in an important segment of the low dose linear region. 


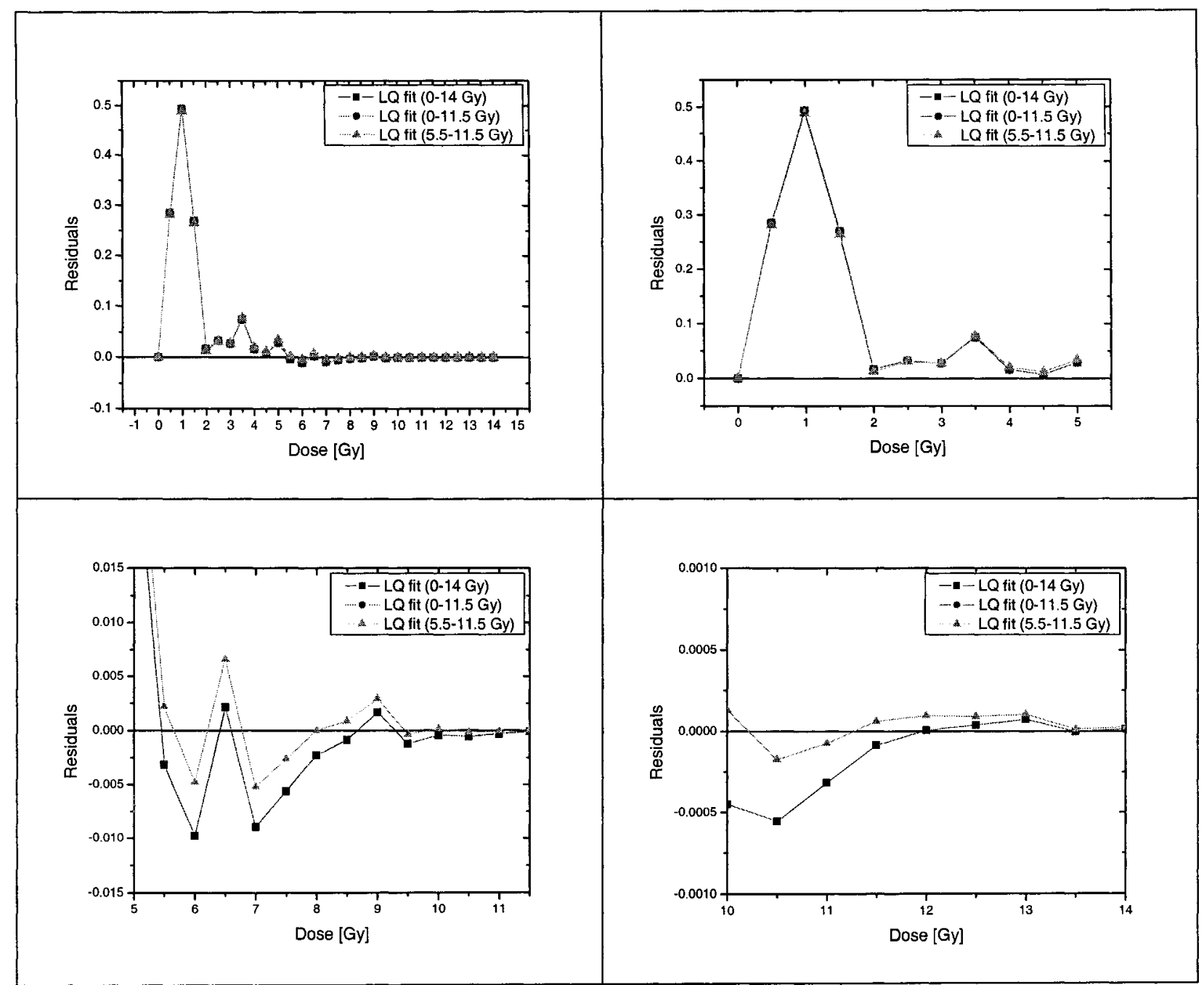

Fig.4.22 Residuals from LQ fit for CP3. The plots show the total range investigated (upper-left corner) and a more detailed behaviour of the same residuals for smaller dose scales: (0-5) Gy in upper-right corner, (5-11) Gy in lower-left corner, (10-14) Gy in lower-right corner 


\subsubsection{Results of data analysis for U373MG cell line}

\subsubsection{Evaluating goodness of fit for U373MG cell line}

The $x^{2} / \mathrm{df}$ behaviour from the $L Q$ fit at different dose ranges is displayed in Fig.4.23 for the U373MG cell line. The graph shows the results for fixing the initial dose at 0,2.5 and 4 Gy while the final dose varies along the $X$-axis.

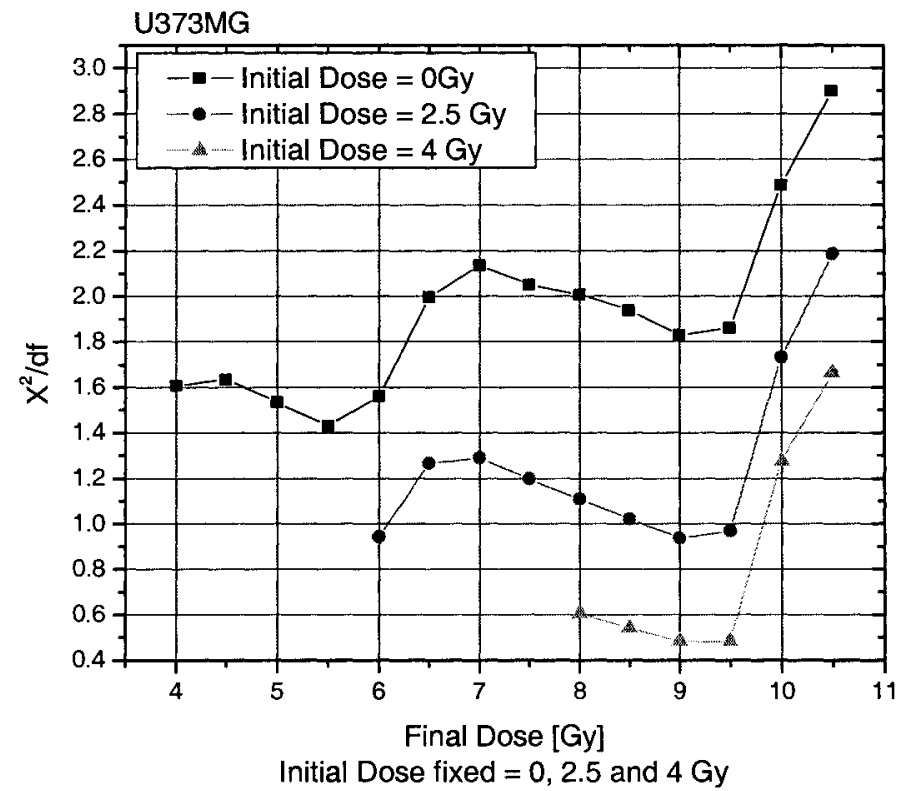

Fig. 4.23 Chi-Square divided by the number of degrees of freedom at different dose ranges for U373MG cell line. Initial dose of the range is fixed at $0,2.5$ and $4 \mathrm{~Gy}$. Final dose of the range varies along the $\mathrm{X}$-axis. 
Figure 4.23 exhibits two steps at 6 and 9.5 Gy in the $X^{2} / d f$ plot when the initial dose is fixed at 0 Gy. Eliminating the first 2.5 Gy the first step is still seen but the values go down again to the same level. The curves are shifted when the data points below 2.5 and 4 Gy are excluded. This feature is expressed in Fig.4.24 as an initial step for doses $\leq 2$ Gy, followed by a decrease of $x^{2} / d f$ values until they finally stabilize around 0.48 at 4 Gy, which mean an improvement of $77 \%$.

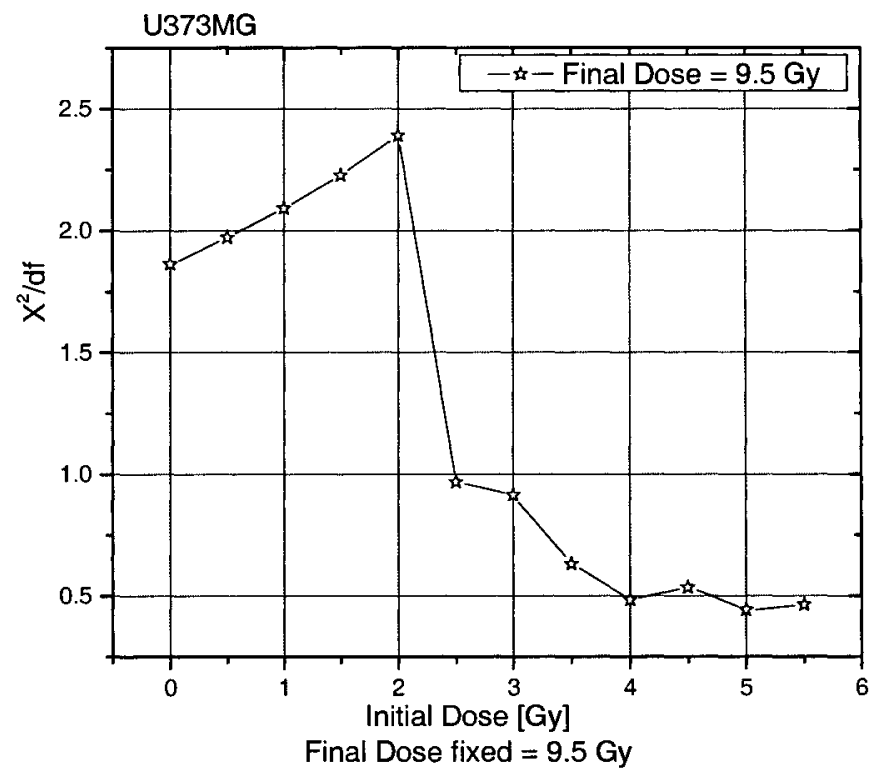

Fig. 4.24 Chi-Square divided by the number of degrees of freedom at different dose ranges for U373MG. Final dose of the range is fixed at $9.5 \mathrm{~Gy}$. Initial dose of the range varies along the $\mathrm{X}$-axis.

The results of testing the LQ hypothesis for a significance level of $5 \%$ for several dose ranges are in Table 4.11. 
Table 4.11 $\mathrm{X}^{2}$-Test for U373MG cell line at several dose ranges. Testing the LQ hypothesis for a significance level of $5 \%$. In the last column the P-value is also shown.

\begin{tabular}{|c|c|c|c|c|c|}
\hline $\begin{array}{l}\text { Dose Range [ } \\
\text { Gy] }\end{array}$ & $d f$ & $\begin{array}{l}\text { T-variable } \\
\mathrm{X}^{2} \text { ' from the fit }\end{array}$ & $\begin{array}{c}\alpha_{\mathrm{sl}}=5 \% \\
\mathrm{X}^{2}(0.95, \mathrm{df})\end{array}$ & $\begin{array}{l}\text { Critical Region } \\
T>x^{2}(0.95, d f)\end{array}$ & P-value \\
\hline $0-10.5$ & 20 & 58.00 & 31.41 & $\mathrm{H}_{0}:$ Rejected & $1.45 \times 10^{-5}$ \\
\hline $0-10$ & 19 & 47.27 & 30.14 & $\mathrm{H}_{0}:$ Rejected & 0.0003 \\
\hline $0-9.5$ & 18 & 33.52 & 25.00 & $\mathrm{H}_{0}:$ Rejected & 0.0144 \\
\hline $0-8$ & 15 & 30.11 & 25.00 & $\mathrm{H}_{0}:$ Rejected & 0.0115 \\
\hline $2-10.5$ & 16 & 57.85 & 26.30 & $\mathrm{H}_{0}:$ Rejected & $2.00 \times 10^{-6}$ \\
\hline $2-10$ & 15 & 47.19 & 25.00 & $\mathrm{H}_{0}:$ Rejected & $3.44 \times 10^{-5}$ \\
\hline $2-8$ & 11 & 29.98 & 19.68 & $\mathrm{H}_{0}:$ Rejected & 0.0016 \\
\hline $4-10.5$ & 12 & 19.99 & 21.03 & $\mathrm{H}_{0}:$ Not rejected & 0.0672 \\
\hline $4-10$ & 11 & 14.01 & 19.68 & $\mathrm{H}_{0}:$ Not rejected & 0.23 \\
\hline $4-9.5$ & 10 & 4.83 & 18.31 & $\mathrm{H}_{0}:$ Not rejected & 0.90 \\
\hline $4-8$ & 7 & 4.24 & 14.07 & $\mathrm{H}_{0}:$ Not rejected & 0.75 \\
\hline
\end{tabular}

The table shows that the LQ model for a significance level of $5 \%$ is rejected unless the low-dose region is excluded. This characteristic differentiates the U373MG cell line from the other cell lines presented before where in order for the LQ hypothesis not to be rejected the high dose region cannot be included and less influence was seen from low dose region. However, for U373MG the test for a $5 \%$ significance level does not show any difference if the high dose region is included or not and although the total measured range is shorter, the LQ hypothesis is always rejected when the low dose data are included. The hypothesis cannot be rejected only if the low dose region is left out of the fit and also an increment of the P-values is observed. 


\subsubsection{Monte Carlo Simulation for U373MG cell line}

Monte Carlo analysis for the U373MG cell line is shown in Figs. 4.25 and 4.26 for the T-and P-value distributions. It is clear that the residuals are not $X^{2}$ distributed for the total dose range from 0 to $10.5 \mathrm{~Gy}$. Reducing the final dose of the range and keeping the initial dose equal zero, the Monte Carlo T-distribution starts moving closer to the expected theoretical distribution. The best result is reached for the final dose equal to 9.5 Gy; further reduction in the final dose will not noticeably improve the concordance between the actual and the expected distributions for the number of degrees of freedom involved. See the graph in the ranges from 0 to $10.5,9.5,8$ and 6 Gy.

The next displayed cases in Fig.4.25 are in the range from 2 and 4 Gy to 9.5 Gy. There is no improvement comparing the results in the range between 0 and 9.5 Gy with the range from 2 to $9.5 \mathrm{~Gy}$. The best concordance is reached for the fit between 4 and 9.5 Gy. The expectations of the theoretical $x^{2}$-distribution and the expectation value obtained from Monte Carlo simulation are shown in Table 4.12 for the U373MG cell line.

Fig.4.26 shows the P-value distribution, displaying that the best agreement between LQ model and the survival data is found in the region from 4 to $9.5 \mathrm{~Gy}$. 
Table 4.12 Expectations for the theoretical and $M C X^{2}$ - distributions at several dose ranges for U373MG cell line. The difference is also displayed in the last column.

\begin{tabular}{|c|c|c|c|}
\hline Dose Range [Gy] & Expectation for $X^{2}$ th & Expectation for $X^{2}$ MC & Difference \\
\hline $0-10.5$ & 20 & 78 & 58 \\
\hline $0-10$ & 19 & 66 & 47 \\
\hline $0-9.5$ & 18 & 51 & 33 \\
\hline $0-9$ & 17 & 48 & 31 \\
\hline $0-8$ & 15 & 45 & 30 \\
\hline $0-6$ & 11 & 28 & 17 \\
\hline $2-9.5$ & 14 & 47 & 33 \\
\hline $3-9.5$ & 12 & 23 & 11 \\
\hline $4-9.5$ & 10 & 15 & 5 \\
\hline $4.5-9.5$ & 9 & 14 & 5 \\
\hline
\end{tabular}




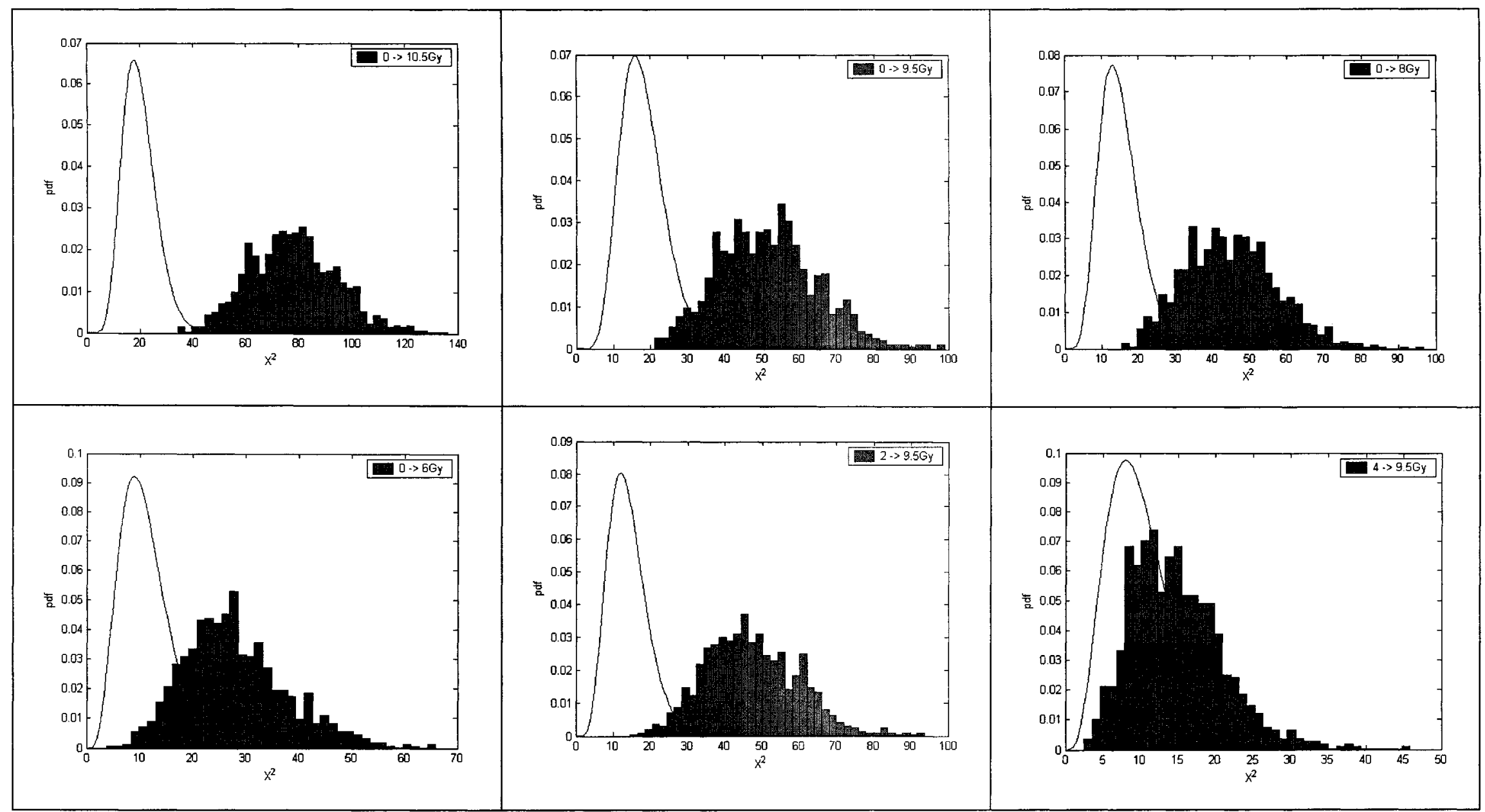

Fig. 4.25 Plots of Chi-Square distribution from Monte Carlo experiment for U373MG in dark bars and the theoretical expected Chi-Square distribution in solid lines. The graphs show the distributions for different dose ranges (see the upper right corner of each graph). 


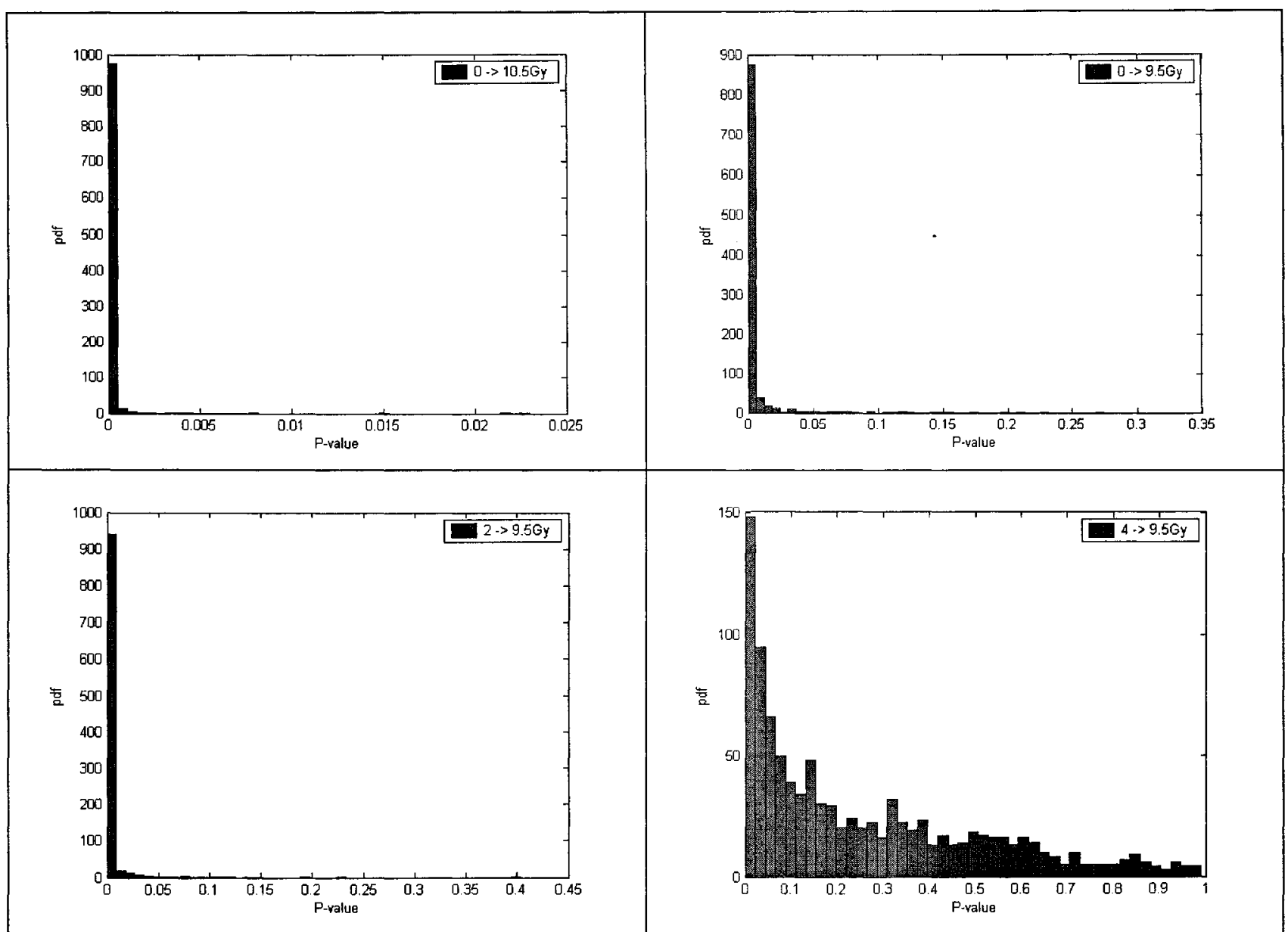

Fig. 4.26 P-value distribution from Monte Carlo experiment at different dose ranges for U373MG. One can see an evident difference among the different dose ranges although the complete flat distribution for P-value is not achieved. 


\subsubsection{Estimated parameter for U373MG cell line.}

The least square estimates of the LQ fit to the survival data for several dose ranges are shown in Figs. 4.27 and 4.28.

The LQ parameters $\alpha$ and $\beta$ have big uncertainties at low dose range for the U373MG cell line. In general $\alpha$ is larger than $\beta$ but the coefficients are not known accurately. This region is followed by an increase of $\alpha$ and a decrease of $\beta$ showing their anti-correlation. The values seem to become stable between 7 and 9.5 Gy especially if the low dose data are not included. The curve starts bending again when the high dose points are retained. The figures also show that the

plots are also shifted one from the other ones when the first 2.5 and 4 Gy are excluded. 


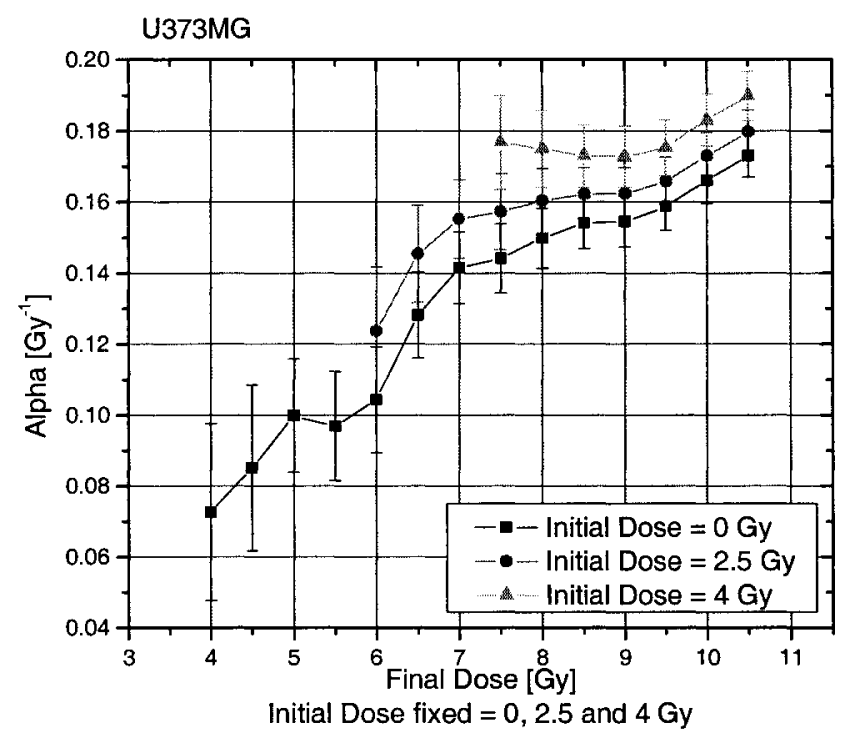

Fig.4.27 Alpha parameters for the LQ model obtained from LSM. Initial dose of the range is fixed at 0, 2.5 and 4 Gy. U373MG cell line.

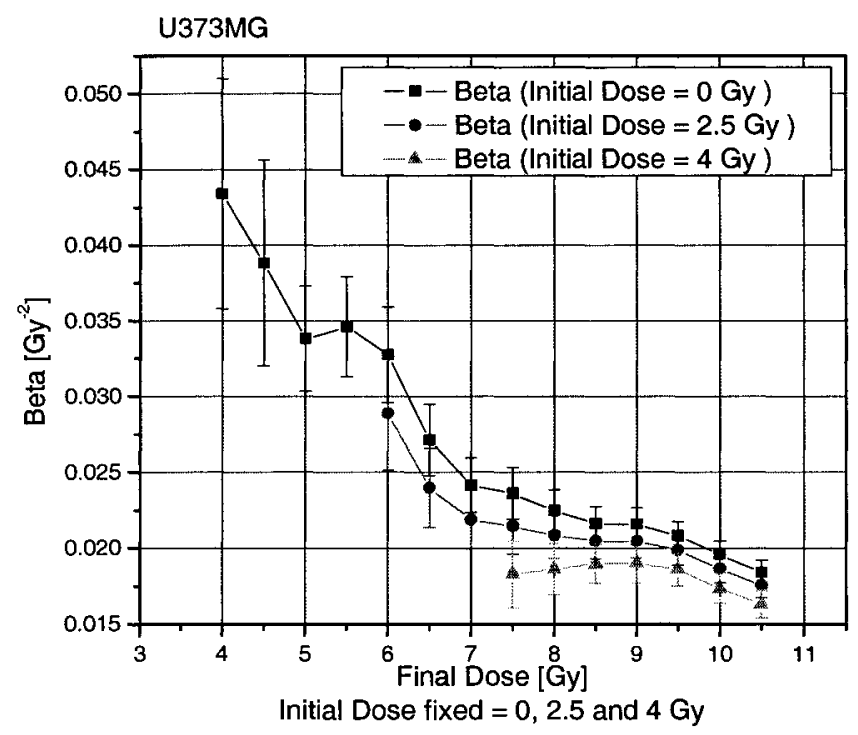

Fig.4.28 Beta parameters for the LQ model obtained from LSM. Initial dose of the range is fixed at $0,2.5$ and 4 Gy. U373MG cell line. 
Table 4.13 summarizes some numerical values of $\alpha$ and $\beta$ parameters and $x^{2}-$ statistics for several dose ranges presented before. In the Fig.4.29 the data and the $L Q$ fits are plotted.

Table 4.13 The parameters $\alpha$ and $\beta$ from LQ model least square fit to the U373MG survival data. Their corresponding standard deviation $\sigma_{\alpha}$ and $\sigma_{\beta}$. The $x^{2} / d f$ statistics from the fit and the correlation coefficient between $\alpha$ and $\beta$.

\begin{tabular}{|l|c|c|c|c|c|c|}
\hline Dose Range [ Gy] & $\begin{array}{c}\text { Alpha } \\
{\left[\mathrm{Gy}^{-1}\right]}\end{array}$ & $\begin{array}{r}\sigma_{\alpha} \\
{\left[\mathrm{Gy}^{-1}\right]}\end{array}$ & $\begin{array}{c}\text { Beta } \\
{\left[\mathrm{Gy} \mathrm{y}^{-2}\right]}\end{array}$ & $\begin{array}{c}\sigma_{\beta} \\
{\left[\mathrm{Gy}^{-2}\right]}\end{array}$ & $\mathrm{x}^{2} / \mathrm{df}$ & $\rho_{\alpha \beta}$ \\
\hline $\begin{array}{l}\text { Total range } \\
0-10.5\end{array}$ & 0.173 & 0.006 & 0.0184 & 0.0008 & 2.90 & -0.97 \\
\hline $\begin{array}{l}\text { Excluding high doses } \\
0-9.5\end{array}$ & 0.159 & 0.007 & 0.0208 & 0.0010 & 1.86 & -0.97 \\
\hline $\begin{array}{l}\text { Excluding high and very low doses } \\
2.5-9.5\end{array}$ & 0.166 & 0.007 & 0.0199 & 0.0010 & 0.97 & -0.97 \\
\hline $\begin{array}{l}\text { Best LQ fit range found } \\
4-9.5\end{array}$ & 0.175 & 0.008 & 0.0186 & 0.0011 & 0.48 & -0.98 \\
\hline
\end{tabular}






Fig.4.29 LQ fit at different dose ranges for U373MG cell line. The LQ models are extrapolated to the total dose range. 


\subsubsection{Discussion}

A decline of fit quality at low doses ( $\leq 2 \mathrm{~Gy}$ ) based on $\mathrm{x}^{2} / \mathrm{df}$ is observed in the cell line. The result is explained by what seems to be an HRS/RR response. Although the data uncertainty in the region is small with relative error of $7 \%$ a further study becomes necessary to ensure the HRS/RR response. This is important, because as in the case of the CHOAA8 cell line the HRS/RR effect has not been detected in human U373MG glioma cells. However, any regional variability from the general trend of the survival cannot be explained by the LQ formalism.

The $x^{2} / d f$ statistic shows that not just the first 2 Gy are responsible and influencing the fit quality as seen in Fig.4.23. The $x^{2} / d f$ plot shifts down when the dose range lower bound is fixed at $4 \mathrm{~Gy}$. This means that eliminating the initial 4 Gy from the fit $x^{2} /$ df quantity will be reduced. This is displayed in Fig.4.24 by a decrease of $x^{2} / \mathrm{df}$ until the values stabilize around $4 \mathrm{~Gy}$. This is also confirmed by the $x^{2}$ test for a $5 \%$ of confidence level.

The Monte Carlo outcome in Table 4.12 also defines 4Gy as the dose where the LQ formalism better fits the data. This is demonstrated by the closest agreement between the theoretical and the actual expectation of T-distribution. See also Figs.4.25 and 4.26 for a graphical representation of $T$ and P-value distributions. The uncertainties in this cell line are smaller compared to the other two lines already discussed; nevertheless, the distributions are not so close to 
the expected, probably due to the bias introduced when assuming the mean as the actual value of the surviving fraction.

These figures also show a threshold around 9.5 Gy strengthened by a clear increment of $x^{2} / \mathrm{df}$ beyond that dose, see Fig. 4.23; and a decrement of observed P-values in Table 4.11.

All the regions seem to have influence in the estimation of $L Q$ parameters and a relatively constant segment is seen at the middle dose coinciding with the region where LQ model works well.

The residuals between the LQ model and the experimental survival curve are displayed in Fig. 4.30 supporting the idea of non-LQ behaviour for low doses. In the middle doses there is not a clear difference about the random behaviour of the residuals between the fits from 0 to $9.5 \mathrm{~Gy}$ and from 4 to $9.5 \mathrm{~Gy}$. The high dose region cannot be well fitted even though high dose data are included.

In conclusion, the LQ formalism seems to work better in the middle region between 4 and 9.5 Gy for the U373MG data set presented here. Trying to establish and define a possible linear region will need a more careful analysis, as was said before, because of the influence of a possible HRS/RR effects at the very low dose region. In addition, this difficulty is emphasized by the shortness of the linear region. 


\subsubsection{Results of data analysis for DU145 cell line}

\subsubsection{Evaluating goodness of fit for DU145 cell line}

Chi-square statistics divided by the number of degree of freedom $\left(x^{2} / d f\right)$ from the LQ fit are plotted in Fig.4.31 for different dose ranges for the DU145 cell line. The figure 4.31 shows a few cases fixing the initial dose at $0,2,4$ and 6 Gy while the final dose varies along the $\mathrm{X}$-axis of the graph.

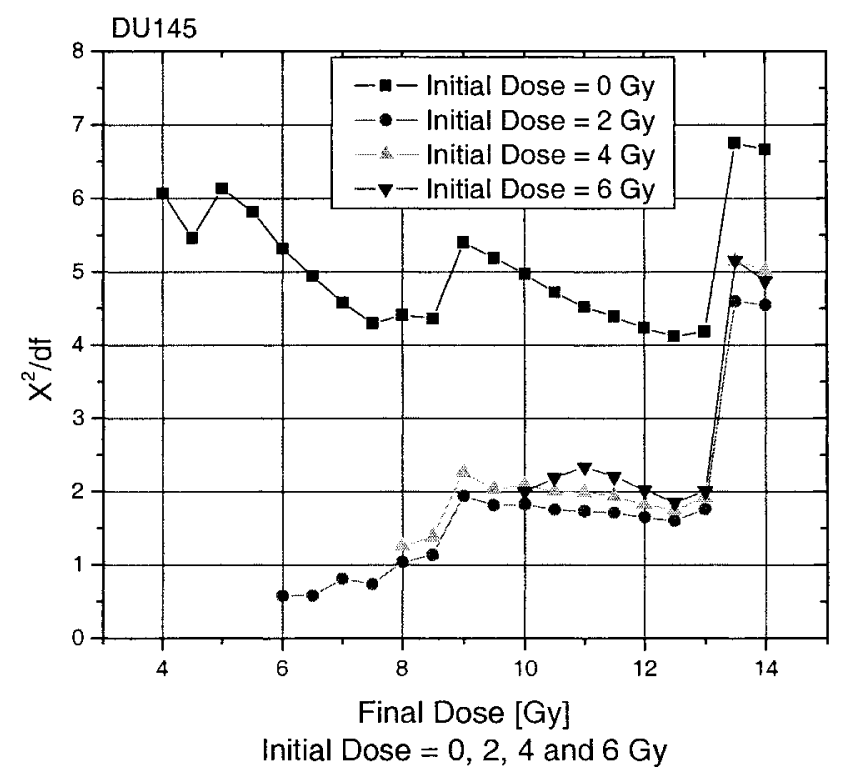

Fig. 4.31 Chi-Square divided by the number of degrees of freedom at different dose ranges for DU145 cell line. Initial dose of the range is fixed at $0,2,4$ and $6 \mathrm{~Gy}$. Final dose of the range varies along the $\mathrm{X}$-axis. 
The figure shows the variability of $x^{2} / \mathrm{df}$ depending on dose range used in the fit. It is clear that including data for dose $<2$ Gy will yield values of $X^{2} /$ df much larger than if the low dose data are removed. However, further exclusion does not exhibit considerable difference. There are two steps evident at 7.5 and $13 \mathrm{~Gy}$, regardless of the lower bound of the range used in the fit.

The influence of low dose data is also shown in Fig.4.32 where the upper bound or final dose was fixed at 7.5 and 13 Gy while the lower bound is changing in the X-axis.

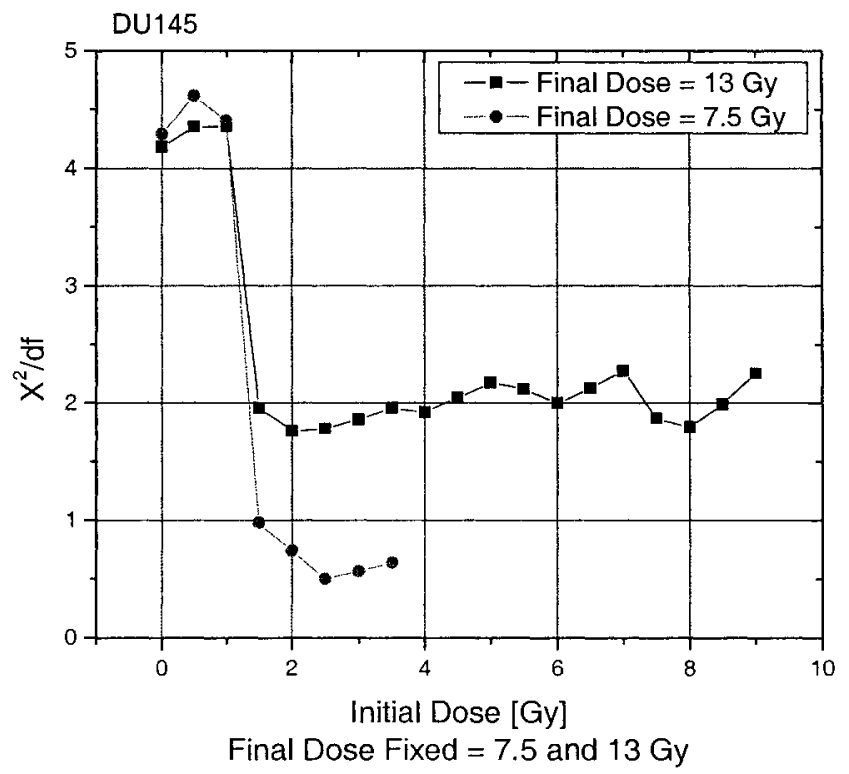

Fig. 4.32 Chi-Square divided by the number of degrees of freedom at different dose ranges for DU145. Final dose of the range is fixed at 7.5 and $13 \mathrm{~Gy}$. Initial dose of the range varies along the $\mathrm{X}$-axis. 
There is a clear improvement when the initial $1 \mathrm{~Gy}$ are left out dropping $x^{2} / \mathrm{df}$ statistics by about $54 \%$ fixing the upper bound of the range at 13 Gy and about $87 \%$ eliminating the first 2.5 Gy and the last 6.5 Gy.

The results of testing the LQ hypothesis for a significance level of $5 \%$ for several dose ranges are shown in Table 4.14.

Table $4.14 \mathrm{x}^{2}$-Test for DU145 cell line at several dose ranges. Testing the LQ hypothesis for a significance level of $5 \%$. In the last column the P-value is also shown.

\begin{tabular}{|c|c|c|c|c|c|}
\hline $\begin{array}{c}\text { DoseRange } \\
\text { [ Gy] }\end{array}$ & df & $\begin{array}{c}\text { T-variable } \\
\mathrm{x}^{2} \text { from the fit }\end{array}$ & $\begin{array}{c}\alpha_{\mathrm{sl}}=5 \% \\
\mathrm{x}^{2}(0.95, \mathrm{df})\end{array}$ & $\begin{array}{c}\text { Critical Region } \\
\mathrm{T}>\mathrm{x}^{2}(0.95, \mathrm{df})\end{array}$ & $\mathrm{P}$-value \\
\hline $0-14$ & 27 & 179.98 & 40.11 & $\mathrm{H}_{0}:$ Rejected & 0 \\
\hline $0-12$ & 23 & 97.39 & 35.17 & $\mathrm{H}_{0}:$ Rejected & $3.96 \times 10^{-11}$ \\
\hline $0-10$ & 19 & 94.36 & 30.14 & $\mathrm{H}_{0}:$ Rejected & $5.56 \times 10^{-12}$ \\
\hline $0-8$ & 15 & 66.11 & 25.00 & $\mathrm{H}_{0}:$ Rejected & $2.18 \times 10^{-08}$ \\
\hline $0-6$ & 11 & 58.43 & 19.68 & $\mathrm{H}_{0}:$ Rejected & $1.81 \times 10^{-08}$ \\
\hline $2-14$ & 23 & 104.51 & 35.17 & $\mathrm{H}_{0}:$ Rejected & $2.32 \times 10^{-12}$ \\
\hline $2-12$ & 19 & 31.33 & 30.14 & $\mathrm{H}_{0}:$ Rejected & 0.0372 \\
\hline $2-10$ & 15 & 27.42 & 25.00 & $\mathrm{H}_{0}:$ Rejected & 0.03 \\
\hline $2-8$ & 11 & 11.41 & 19.68 & $\mathrm{H}_{0}:$ Not rejected & 0.41 \\
\hline $2-6$ & 7 & 4.04 & 14.07 & $\mathrm{H}_{0}:$ Not rejected & 0.78 \\
\hline $4-14$ & 19 & 95.39 & 30.14 & $\mathrm{H}_{0}:$ Rejected & $3.63 \times 10^{-12}$ \\
\hline $4-12$ & 15 & 27.52 & 25.00 & $\mathrm{H}_{0}:$ Rejected & 0.02 \\
\hline $4-10$ & 11 & 22.94 & 19.68 & $\mathrm{H}_{0}:$ Rejected & 0.02 \\
\hline
\end{tabular}

As it was observed in Fig.4.31, Table 4.14 shows that the first 2 Gy are decisive in the results of the $x^{2}$-test for a $5 \%$ significance level. Including the first $2 \mathrm{~Gy}$ no matter what is the upper bound of the range the LQ model can be safely ruled out due to the very small observed P-values. In addition, it is observed that 
including high dose data is also a determinant in the LQ hypothesis rejection. According to this test the $L Q$ model cannot be rejected when the region is included within the range between 2 and 8 Gy.

\subsubsection{Monte Carlo Simulation for DU145 cell line}

The graphical measure of the goodness of fit by plotting the T-variable and Pvalue distributions from one thousand similar experiments simulated using Monte Carlo technique is shown in Figs.4.33 and 4.34.

It is clear that the residuals are far away from $x^{2}$-distribution for the total dose range from 0 to $14 \mathrm{~Gy}$. Reducing the final dose of the range and keeping the initial dose equal to zero, the Monte Carlo distribution starts moving just a little bit closer to the expected theoretical distribution. The best result is reached for the final dose equal to 7.5 Gy; further reduction in the final dose will not noticeably improve the concordance between the actual and the expected distributions for the number of degrees of freedom involved. See the graph in the ranges from 0 to $14,13,7.5$ and 6 Gy.

The next two cases displayed in Fig.4.33 are in the range from 1 and 2.5 to 7.5 Gy. The best concordance is reached for the fit between 2.5 and 7.5 Gy. Increasing the lower bound of the range more than 2.5 Gy does not substantially improve the agreement. In order to quantify the degree of correspondence 
between both distributions, the expectations of the theoretical $x^{2}$-distribution, and the value obtained from the Monte Carlo simulation, are shown in Table 4.15.

Table 4.15 Expectations for the theoretical and $M C \mathrm{X}^{2}$ - distributions at several dose ranges. The difference is also displayed in the last column.

\begin{tabular}{|l|c|c|c|}
\hline Dose Range & Expectation for $x^{2}$ th & Expectation for $x^{2}$ C & Difference \\
\hline $0-14$ & 27 & 207 & 180 \\
\hline $0-13$ & 25 & 130 & 105 \\
\hline $0-9$ & 17 & 109 & 92 \\
\hline $0-8$ & 15 & 81 & 66 \\
\hline $0-7.5$ & 14 & 74 & 60 \\
\hline $0-7$ & 13 & 73 & 60 \\
\hline $0-6$ & 11 & 70 & 59 \\
\hline $1-7.5$ & 12 & 64 & 52 \\
\hline $2-7.5$ & 10 & 17 & 7 \\
\hline $2.5-7.5$ & 9 & 14 & 5 \\
\hline $3-7.5$ & 8 & 13 & 5 \\
\hline $3.5-7.5$ & 7 & 11 & 4 \\
\hline
\end{tabular}

Figure 4.34 also reveals that the best agreement between the LQ model and the survival data is found in the region from 2.5 to 7.5 Gy. Even though the complete flat distribution for the P-values is not achieved, the graphs exhibit an evident difference among several dose ranges. 


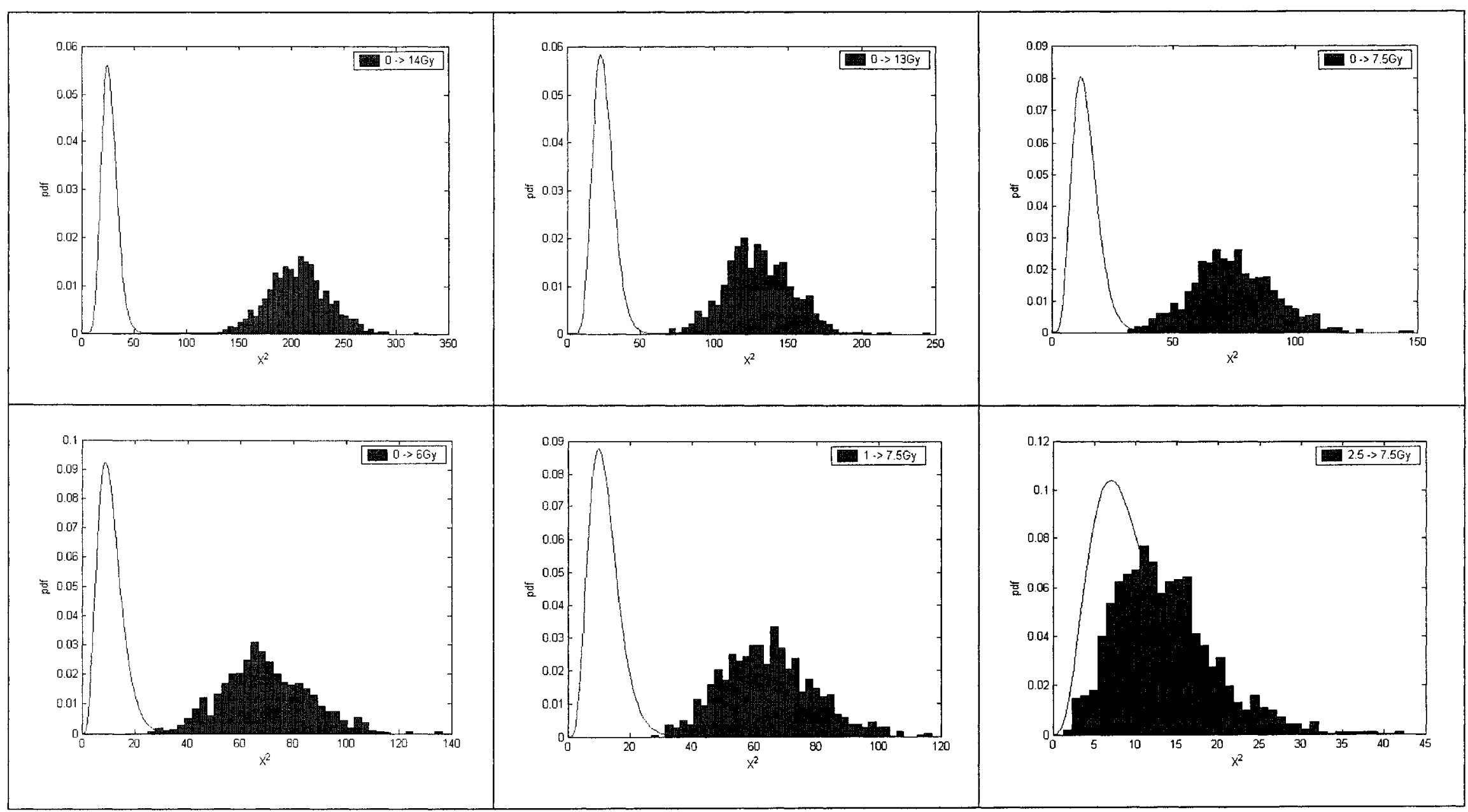

Fig. 4.33 Plots of Chi-Square distribution from Monte Carlo experiment for DU145 in dark bars and the theoretical expected Chi-Square distribution in solid lines. The graphs show the distributions for different dose ranges (see the upper right corner of each graph). 


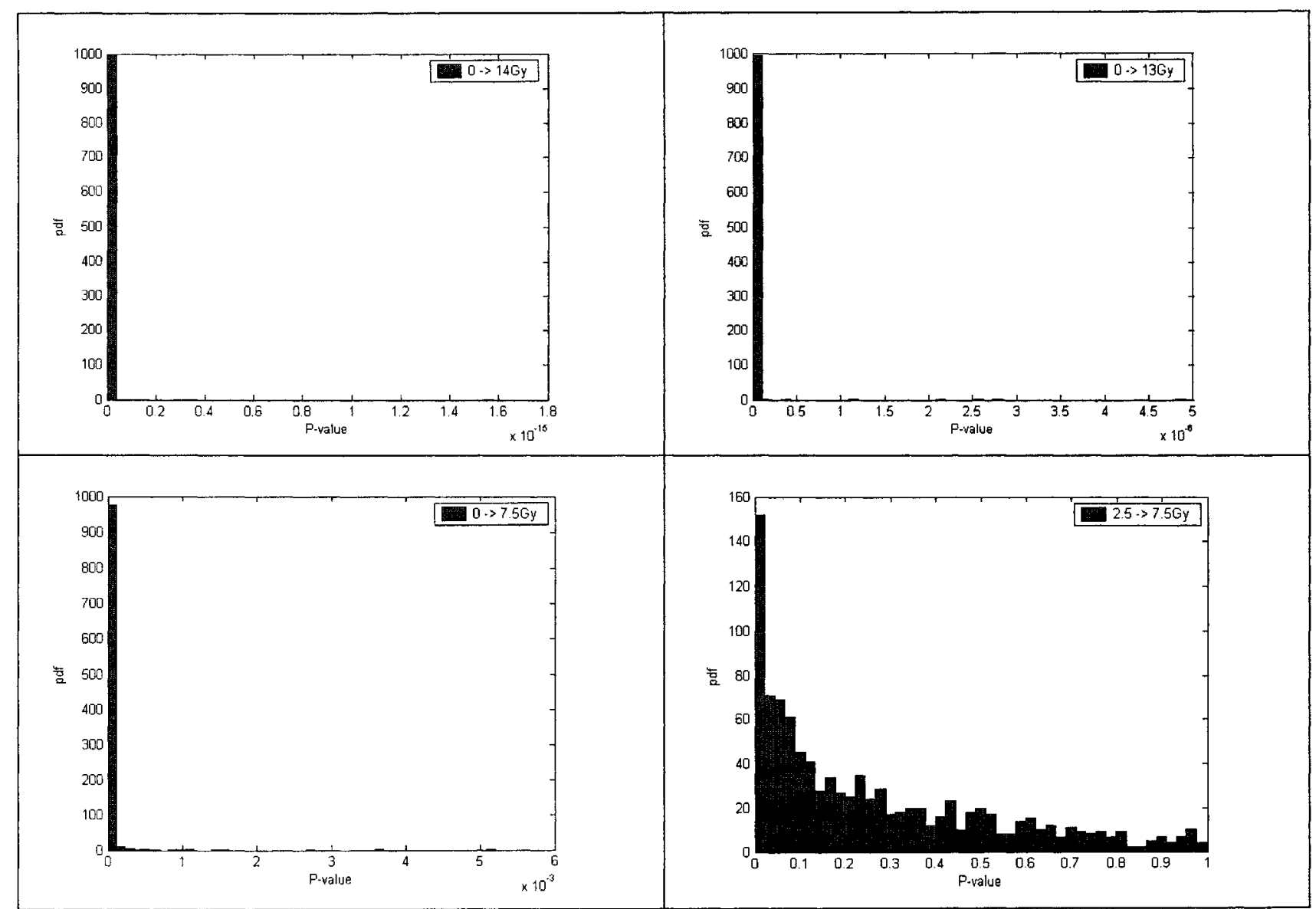

Fig. 4.34 P-value distribution from Monte Carlo experiment at different dose ranges for DU145. One can see an evident difference among the different dose ranges although the complete flat distribution for P-value is not achieved. 


\subsubsection{Estimated parameter for Du145 cell line.}

The least square estimates of the $L Q$ fit to the survival data for several dose ranges are shown in Figs. 4.35 and 4.36.

Both parameters $\alpha$ and $\beta$ have bigger uncertainties at low dose ranges. The parameters $\alpha$ and $\beta$ have about the same value at dose of 4 Gy but the error bars indicate high uncertainty in the estimation. In the curve when the lower bound of the range is fixed at 0 Gy, i.e. low dose data are included, a region of high variability of $\alpha$ and $\beta$ is seen. There is a high increase of $\alpha$ parameter and a high decrease of $\beta$ parameter with the increment of the final dose of the range as expected due to their negative correlation. The estimators appear to stabilize around 6 Gy when a wide portion of the LQ region data is included in the fit. Both Figs. 4.35 and 4.36 do not display a great shift of $\alpha$ or $\beta$ parameters whether the first 2.5 Gy are excluded or not. 


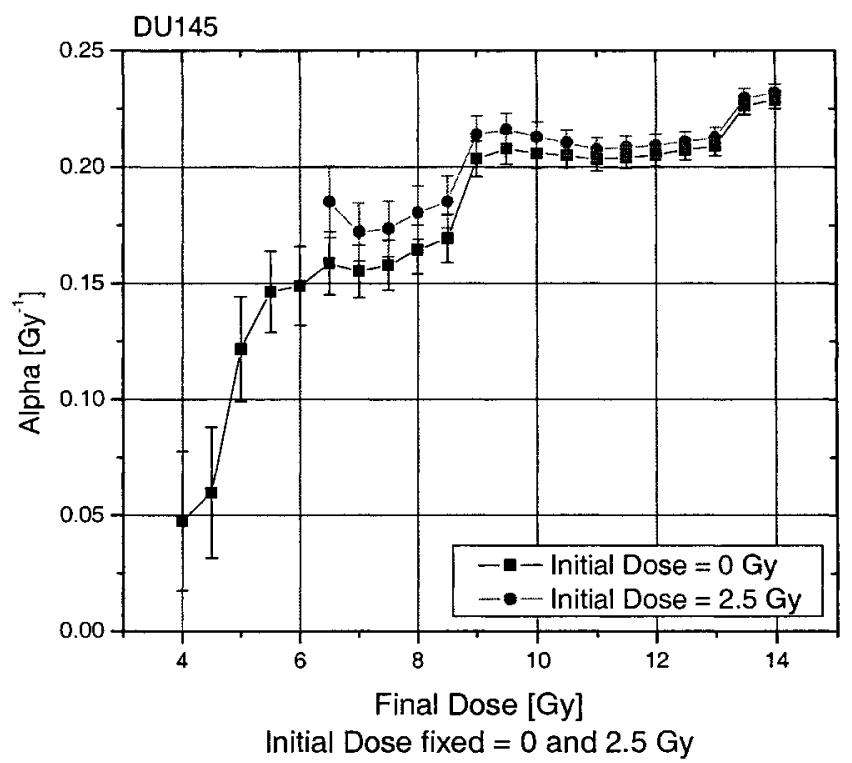

Fig.4.35 Alpha parameters for the LQ model obtained from LSM. Initial dose of the range is fixed at 0 and 2.5 Gy. DU145 cell line.

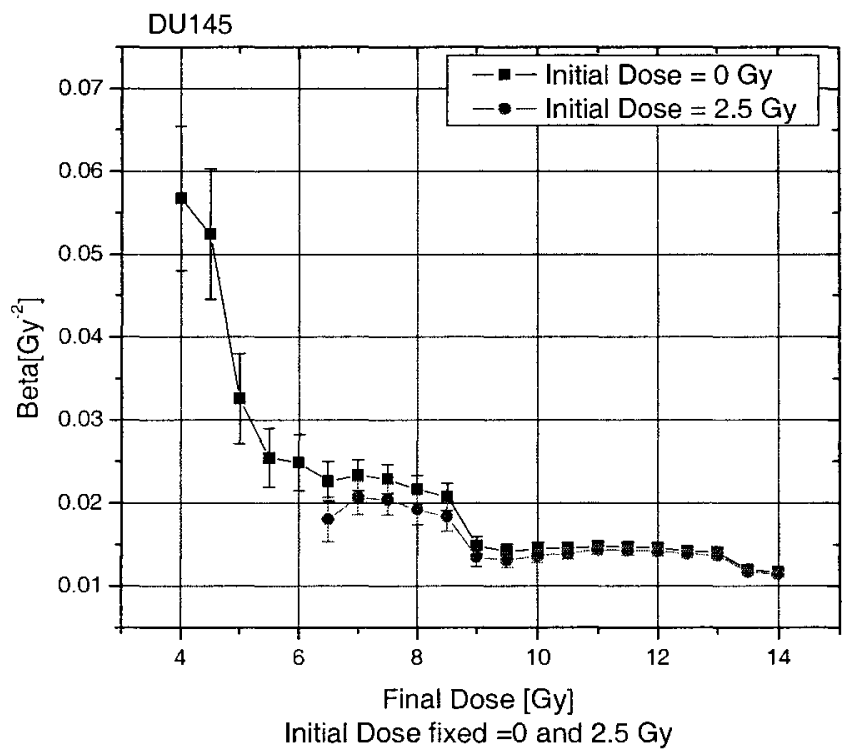

Fig.4.36 Beta parameters for the LQ model obtained from LSM. Initial dose of the range is fixed at 0 and 2.5 Gy. DU145 cell line. 
Table 4.16 summarizes some numerical values of $\alpha$ and $\beta$ parameters and $x^{2}$ statistics for several dose ranges presented before and Fig.4.37 graphically displays the result of the fit.

Table 4.16 The parameters $\alpha$ and $\beta$ from the LQ model least square fit to the DU145 survival data. Their corresponding standard deviation $\sigma_{\alpha}$ and $\sigma_{\beta}$. The $x^{2} / d f$ statistics from the fit and the correlation coefficient between $\alpha$ and $\beta$.

\begin{tabular}{|l|c|c|c|c|c|c|}
\hline Dose Range [Gy] & $\begin{array}{c}\text { Alpha } \\
{\left[\mathrm{Gy}^{-1}\right]}\end{array}$ & $\begin{array}{c}\sigma_{\alpha} \\
{\left[\mathrm{Gy}^{-1}\right]}\end{array}$ & $\begin{array}{c}\text { Beta } \\
{\left[\mathrm{Gy}^{-2}\right]}\end{array}$ & $\begin{array}{c}\sigma_{\beta} \\
{\left[\mathrm{Gy}^{-2}\right]}\end{array}$ & $\mathrm{X}^{2} / \mathrm{df}$ & $\rho_{\alpha, \beta}$ \\
\hline $\begin{array}{l}\text { Total range } \\
0-14\end{array}$ & 0.229 & 0.004 & 0.0117 & 0.0004 & 6.67 & -0.97 \\
\hline $\begin{array}{l}\text { Excluding high doses } \\
0-7.5\end{array}$ & 0.158 & 0.011 & 0.0229 & 0.0017 & 4.29 & -0.98 \\
\hline $\begin{array}{l}\text { Best LQ fit range found } \\
2.5-7.5\end{array}$ & 0.174 & 0.012 & 0.0204 & 0.0019 & 0.51 & -0.99 \\
\hline
\end{tabular}




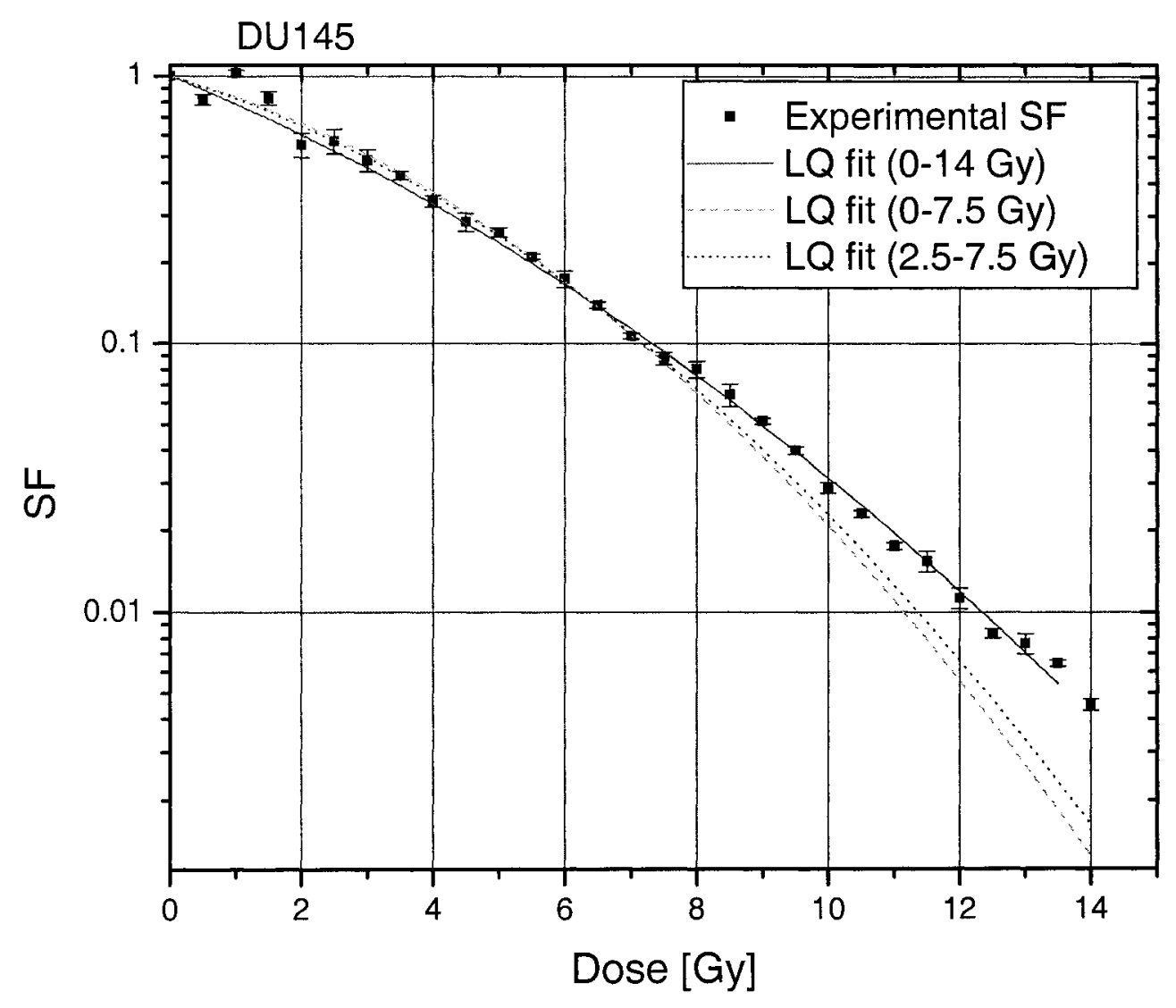

Fig.4.37 LQ fit at different dose ranges for DU145 cell line. The LQ models are extrapolated to the total dose range. 


\subsubsection{Discussion}

Figures 4.31 and 4.32 clearly show several levels in $x^{2} /$ df variability for the DU145 cell line. A decline of fit quality at very low doses ( $<2 \mathrm{~Gy}$ ) based on $x^{2} / \mathrm{df}$ is observed. This result is explained by what seems to be an HRS/RR- type response. However, it is just defined by a few data points making necessary a more detailed analysis in the region. Even though there is no substantial difference in the values by increasing the lower bound up to 4 or 6 Gy, Fig. 4.32 shows a slight improvement for lower bound greater than 2.5 Gy and fixing the upper bound at $7.5 \mathrm{~Gy}$.

In addition, a decline of the fit is seen as well when the high dose data is retained. The step at 13.5 Gy appears due to underestimated errors while the one observed at 9 Gy could be related to the final slope of the survival at higher doses. Therefore, it seems that $L Q$ model works better in the middle dose region within the range from around 2 to $8.5 \mathrm{~Gy}$.

The results from testing the LQ hypothesis for a $5 \%$ significance level indicate that the minimal range where the $L Q$ hypothesis cannot be rejected is approximately within 2 to 8 Gy. See Table 4.14.

Moreover, the outcome of the MC calculations shows that fixing the lower bound at 0 Gy the difference between the expectations of theoretical and MC $x^{2}$ distributions decreases when decreasing the upper bound of the range until it 
reaches the value of 60 at 7.5 Gy. Further reduction of the final dose does not show noticeable change. See the first seven rows in Table 4.15. Now, keeping the upper bound at $7.5 \mathrm{~Gy}$, the table displays that increasing the lower bound the values stabilize at 2.5 Gy with a difference equal 4.52 .

Therefore, it seems that the LQ model works better in the middle dose region within the range from 2.5 to $7.5 \mathrm{~Gy}$. This is confirmed by examination of the residuals in the region for different dose range fits. See Fig.4.38.

The hypothesis of linearity for the low dose region cannot be tested since it is very short compared to the other cell lines studied and matches the presumable HRS/RR region.

An interesting feature coming out after analyzing all cell lines is that there is an increment of the parameter $\alpha$ and a decrease of the parameter $\beta$ when the analysis curve goes from linear to the LQ region for every human tumor cell lines (CP3, U373MG, DU145). Issue which differs for the normal Hampters cell line (CHOAA8). 




Fig.4.38. Residuals from LQ fit for DU145. The plots show the total range investigated (upper-left corner) and a more detailed behaviour of the same residuals for smaller dose scales: (0-5) Gy in upper-right corner, (2-8) Gy in lower-left corner, (8-14) Gy in lower-right corner. 


\subsection{Impact of low dose region in $\alpha / \beta$ ratio}

As introduced before, another important concept in this study and coming from the linear quadratic model is the $\alpha / \beta$ ratio. It represents the dose where the two mechanisms of cell killing, lethal lesions and sub-lethal lesion exchange have equal importance. This can predict how different types of tissue respond to different fractionation schemes. Even though this ratio was calculated from the survival curve fit, it can be used to make relative comparisons among different tissues at various dose ranges.

Table 4.17 presents the LQ regions best fitted in this study. How the $\alpha / \beta$ ratio is influenced by the low dose region is explained further.

Table 4.17 LQ Region for each cell line.

\begin{tabular}{|l|c|c|}
\hline Cell line & Total Range [ Gy] & LQ Region [ Gy] \\
\hline CHOAA8 & $0-16$ & $\sim 5.5-13$ \\
\hline U373MG & $0-10.5$ & $\sim 4-9.5$ \\
\hline CP3 & $0-14$ & $\sim 5.5-11.5$ \\
\hline DU145 & $0-14$ & $\sim 2.5-7.5$ \\
\hline
\end{tabular}

Figures $4.39,4.40,4.41$ and 4.42 show the $\alpha / \beta$ ratio for the studied cell lines. The initial doses were fixed at $0 \mathrm{~Gy}, 2.5 \mathrm{~Gy}$ and at the lowest boundary of the linear quadratic region for every cell line. 


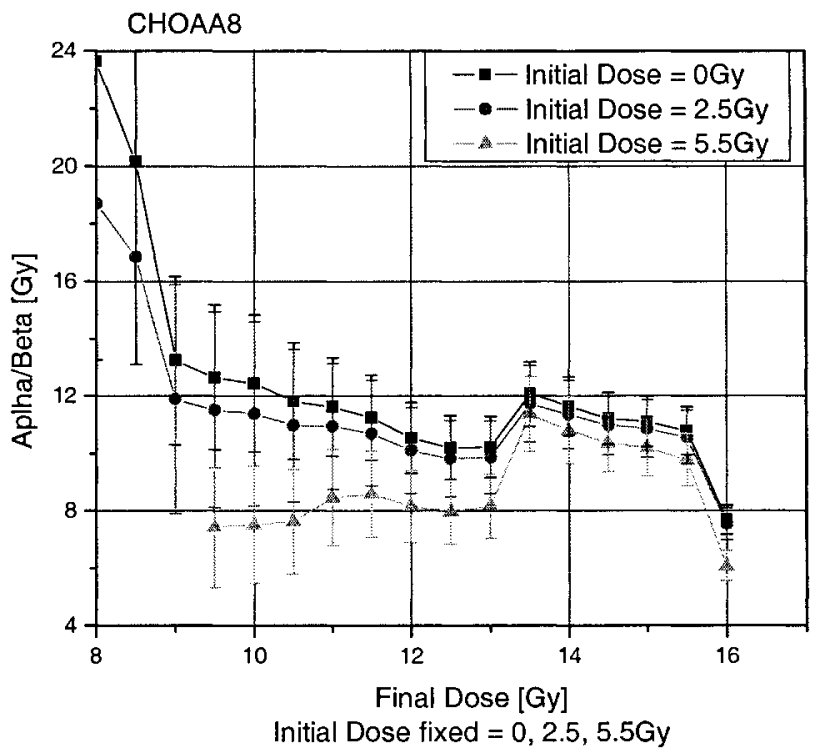

Fig. 4.39 Plot of the $\alpha / \beta$ ratio for CHOAA8 cell line.



Fig. 4.40 Plot of the $\alpha / \beta$ ratio for $\mathrm{CP} 3$ cell line. 


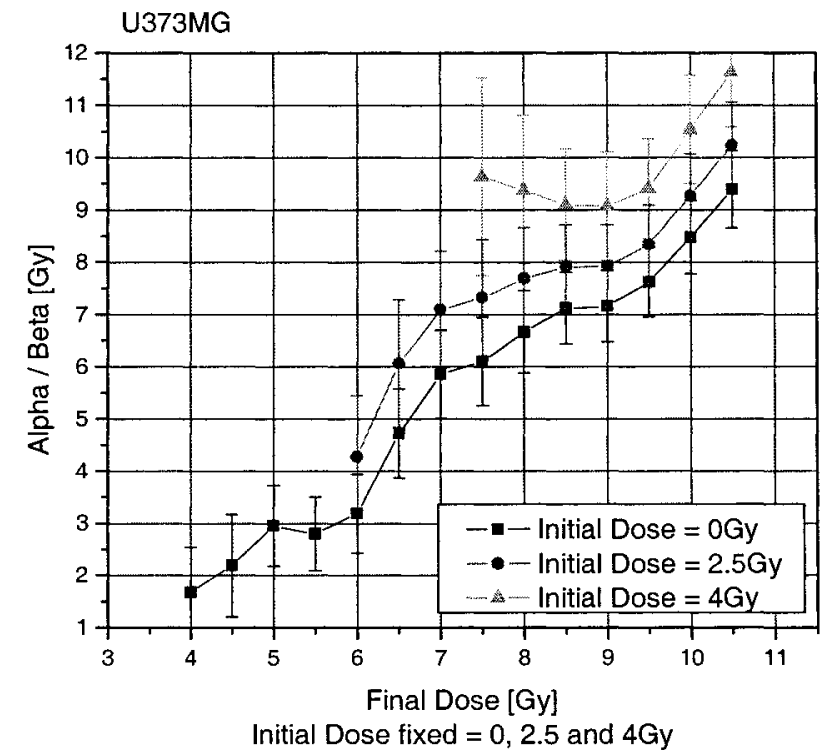

Fig. 4.41 Plot of the $\alpha / \beta$ ratio for U373MG cell line.

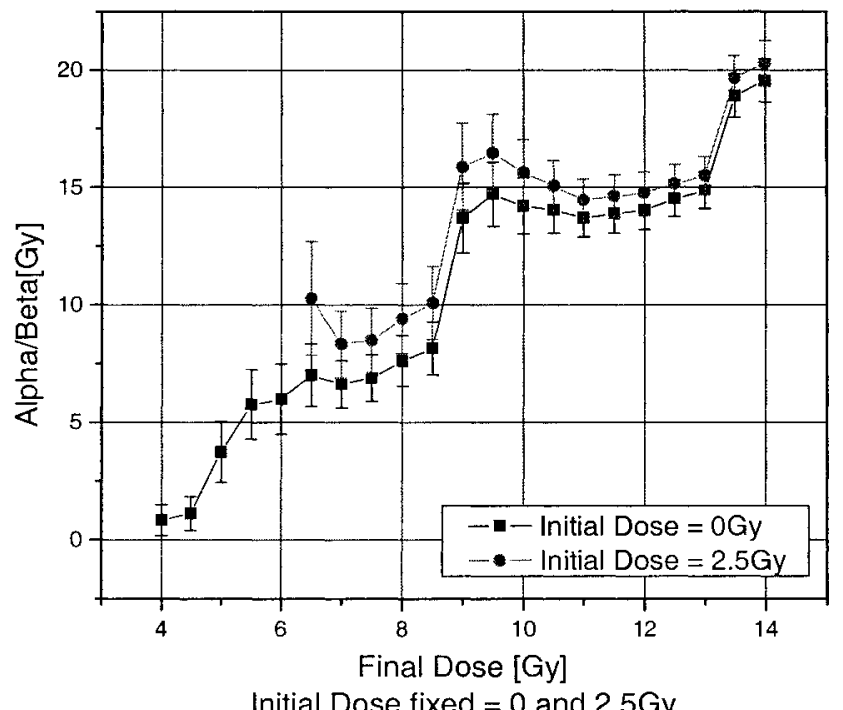

Fig. 4.42 Plot of the $\alpha / \beta$ ratio for DU145 cell line. 
There is a high variability in $\alpha / \beta$ ratio values at the low dose region for all the cell lines studied due to the variability of the parameters $\alpha$ and $\beta$. When an extensive part of the LQ region data is included in the fit the values of the $\alpha / \beta$ ratios appear to stabilize as happened individually with $\alpha$ and $\beta$.

One can see that there is a noticeable difference in the $\alpha / \beta$ ratio by including or excluding low dose data for all studied cell lines in the region of interest, at middle doses.

No big difference is seen in $\alpha / \beta$ values at middle doses if the very low dose data are left out (2 Gy) for CHOAA8 and CP3 cell lines. Even though the very low dose region characterized by HRS/RR and adaptive responses strongly affects the quality of the fit, this is not displayed in the $\alpha / \beta$ ratio results.

The $\alpha / \beta$ ratio is related to the time required for cells to divide and cycle as explained in section 2.1.3. High or low values of this ratio define the tissue response as being early or late response to ionizing radiation. This feature is very important under fractionation scheme in order to control the tumour and spare normal tissues.

The influence of low dose data through the $\alpha / \beta$ ratio is summarized in Fig.4.43. The bar heights exhibit an obvious difference between the values calculated including or excluding low dose data. The percentage differences were found to 
be $42 \%, 40 \%, 23 \%$ and $24 \%$ for the CHOAA8, CP3, U373MG and DU145 cell lines, respectively.

All the results clearly show that the $\alpha / \beta$ ratio is dose range dependent. Although the analyses have been performed for in vitro experiments, we should expect having the same dependence for in vivo clinical studies.

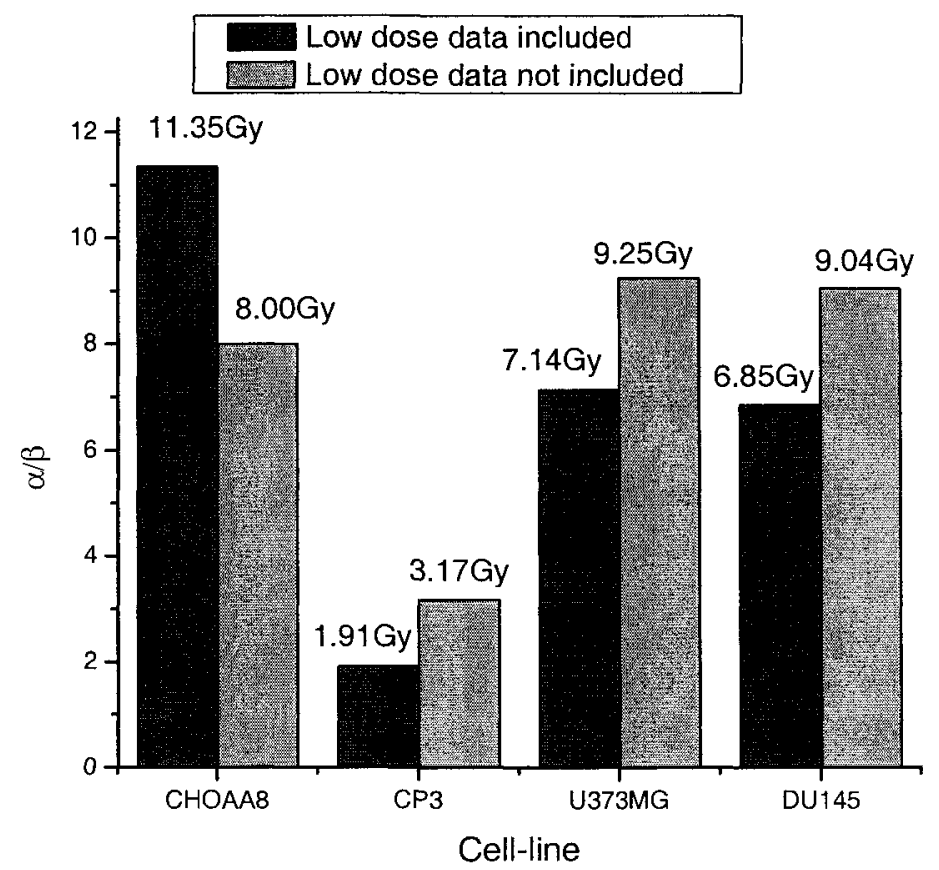

Fig. $4.43 \alpha / \beta$ ratio including and not including low dose data points.

Indeed, the range included in the fit will affect the results of fitted parameters, which just stabilize when the principal LQ region is included in the fit. In addition, the presence of the low dose region that could be related to the linearity of the 
survival curve in that region highly influences the parameters and consequently the value of the $\alpha / \beta$ ratio coming from the LQ approach. 


\section{Chapter 5}

\section{CONCLUSIONS AND FUTURE DIRECTIONS}

\subsection{Conclusions}

Detailed data sets for the desired study from clonogenic assays in a broad range of dose were obtained. The total dose range reached various end-points from 10.5 to 16 Gy depending on the radiosensitivity of the cell line. A dose increment of 0.5 Gy was used over the entire dose range. The four cell lines investigated were: CHOAA8, hamster fibroblast cells; U373MG, human glioblastoma cells; and human prostate carcinoma cell lines CP3 and DU-145. All cell lines showed an influence of multiplicity factor on survival and we corrected the data before any further analysis.

Goodness of fit and MC analysis did exhibit a dependence on dose region. From the general survival curve shape, three main regions were proven to be involved: 
the low dose region, a middle dose region and the high dose region. A drop in the fit quality was found when low doses were included. This could be a result of the linearity in the trend of the survival curve at low doses that will affect the total fit in a range from 0 Gy to the final dose in the linear quadratic region. However, the very low dose region ( 0-2 Gy) strongly influences the decline of the fit quality. This outcome can be explained by the hypersensitive effect observed in CHOAA8 and U373MG data and an adaptive type response in CP3 cell line.

The LQ behaviour characterizes the survival curves in the middle dose region, which is demonstrated by a clear improvement of goodness of fit and MC analyses (Table 4.17). The fit worsening at high doses showed as expected, that the LQ model cannot explain this region. Exactness of region delimitation is affected by hypersensitivity, adaptive response at very low doses and data uncertainties.

The dependence of survival curve trend on dose range did influence the $\alpha$ and $\beta$ parameters and the $\alpha / \beta$ ratio. The impact of including low dose data is shown through $\alpha / \beta$ ratios showing relative differences of $42,40,23$ and $24 \%$ for CHOAA8, CP3, U373MG and DU145, respectively. 


\subsection{Future Directions}

In this study, dose dependence of survival behaviour, LQ response, fitted parameters and $\alpha / \beta$ ratio has been demonstrated. The statements can be drawn from the experimental and analyzed results discussed in chapter 4 . Some of the results pointed out the final conclusions presented above and other ones lead to further questions. The first question is related to the association of the low dose region with the expected linear region. It is hard to define the linear region due to its shortness, superposition of HRS/RR and adaptive type responses, and data uncertainty. The second question is how to verify the existence of HRS/RR and adaptive responses. In both cases, more detailed data sets are necessary. Another important question is how to include the high dose region in the LQ model by adding more parameters to the model. However, this region is highly affected by the precision of the clonogenic survival technique.

Therefore, the LQ model needs to be developed further in order to explain the high dose region ${ }^{45,63}$. One possibility might be the Linear-Quadratic-Linear model $(L Q L)^{45}$ developed by Carlone, which is an extension of the LQ. In order to model the low dose region, more detailed data have to be obtained to identify the survival curve dose dependence on $\alpha, \mathrm{HRS} / \mathrm{RR}$ and adaptive type responses. 


\section{References}

1. E. J. Hall, Radiobiology for radiologist, Fourth Edition, Lippincott Williams \& Wilkins, Philadelphia (2000).

2. D. T. Goodhead, "Spatial and temporal distribution of energy," Health Physics, 55, 231-240 (1988).

3. G.G. Steel, G.E. Adams and A. Horwich, The biological basis of radiotherapy, Second Edition, Elsevier Science Publishers B.V., Amsterdam (1989).

4. T.T. Puck and P. I. Marcus, "Action of X-rays on mammalian cells," J. Exp. Med. 103, 653-666 (1956).

5. J.H. Peacock, M.R. De Almodovar, T.J. Mcmillan, and G.G. Steel, "The nature of the initial slope of radiation cell survival curves," Brit. J. Radiol. 24, S57-S60 (1992).

6. C.A. Tobias, "The repair-misrepair model in radiobiology: comparison to other models," Radiat. Res. 8, S77-95 (1985).

7. S.B. Curtis, "Lethal and potentially lethal lesions induced by radiation--a unified repair model," Radiat. Res. 106, 252-270 (1986). 
8. R.K. Sachs, P. Hahnfeldt, and D.J. Brenner, "Review: The link between low-LET dose-response relations and the underlying kinetics of damage production/repair/misrepair," Int. J. Radiat. Biol. 72, 351-374 (1997).

9. M.J. Marchese, M. Zaider and E.J. Hall, "Dose-rate effects in normal and malignant cells of human origin," Br. J. Radiol. 60, 573-576 (1987).

10.P.J. Deschavanne, B. Fertil, N. Chavaudra, and E.P. Malaise, "The relationship between radiosensitivity and repair of potentially lethal damage in human tumor cell lines with implications for radioresponsiveness," Radiat. Res. 122, 29-37 (1990).

11.G.W. Barendsen, "Parameters of linear-quadratic radiation dose-effect relationships, dependence on LET and mechanisms of reproductive cell death," Int. J. Radiat. Biol. 71, 649-655 (1997).

12.J.D. Chapman, "Single-hit mechanism of tumor cell killing by radiation" Int. J. Radiat. Biol., 79(2), 71-81, (2003).

13. M. Zaider, "Evidence of a neutron RBE of $70( \pm 50)$ for solid-tumor induction at Hiroshima and Nagasaki and its implications for assessing the effective neutron quality factor," Health. Phys. 61, 631-636 (1991).

14.D.J. Brenner, P. Hahnfeldt, S.A. Amundson and R. K. Sachs, "Interpretation of inverse dose-rate effects for mutagenesis by sparsely ionizing radiation," Int. J. Radiat. Biol. 70, 447-458 (1996).

15.E. Schmid, H. Roos, G. Rimpl and M. Bauchinger, "Chromosome aberration frequencies in human lymphocytes irradiated in a multi-layer array by protons with different LET," Int. J. Radiat. Biol. 72, 661-5 (1997).

16. K. H. Chadwick and H. P. Leenhouts, "A molecular theory of cell survival," Phys. Med. Biol. 18, 78-87 (1973).

17.H.R. Whithers, "Biologic basis for altered fractionation schemes," Cancer 55, 2086-2095 (1985).

18. H.D. Thames and J.H. Hendry, Fractionation in radiotherapy, Taylor and Francis, London (1987). 
19.J.F. Fowler, "The linear-quadratic formula and progress in fractionated radiotherapy," Brit. J. Radiol. 62, 679-694 (1989).

20.B. Jones, L.T. Tan and R.G. Dale, "Derivation of the optimum dose per fraction from the linear quadratic model," Br. J. Radiol. 68, 894-902 (1995).

21.J.H. Hendry, S.M. Bentzen, R.G. Dale, J.F. Fowler, T.E. Wheldon, B. Jones, A.J. Munro, N.J. Slevin and A.G. Robertson, "A modelled comparison of the effects of using different ways to compensate for missed treatment days in radiotherapy," Clin. Oncol. (Royal College of Radiologists) 8, 297-307 (1996).

22.C. Robertson, A.G. Robertson, J.H. Hendry, S.A. Roberts, N.J. Slevin and W.B. Duncan, "Similar decreases in local tumor control are calculated for treatment protraction and for interruptions in the radiotherapy of carcinoma of the larynx in four centers," Int. J. Radiat. Oncol. Biol. Phys. 40, 319-329 (1998).

23.D. J. Brenner and E.J. Hall, "Conditions for the equivalence of continuous to pulsed low dose rate brachytherapy," Int. J. Radiat. Oncol. Biol. Phys. 20, 181-190 (1991).

24.D. Dodds, R.P. Symonds, C. Deehan, T. Habeshaw, N.S. Reed and D.W. Lamont, "A linear quadratic analysis of gynaecological brachytherapy," Clin. Oncol. (R. Coll. Radiol.) 8, 90-96 (1996).

25. O. Algan, C.C. Stobbe, A. M. Helt, G. E. Hanks, and J.D. Chapman, "radiatioan inactivation of human prostate cancer cells: the roll of apoptosis," Radiat. Res. 146, 267-275 (1996).

26.D. Bartkowiak et al.,"Cell cycle and growth response of $\mathrm{CHO}$ cells to Xirradiation: Threshold-free repair at low doses," Int. J. Radiat. Oncol. Biol. Phys., 50, 221-227 (2001).

27.R. Sachs, "The mechanistic basis of the linear-quadratic formalism," Medical Physics 75, 2071-2073 (1998). 
28. M. Kellerer and H. H. Rossi, "The theory of dual radiation action," Current Topics in Radiation Research Quarterly 8, 85-158 (1972).

29.M. Cornforth and J. Bedford, "A quantitative comparison of potentially lethal damage repair and the rejoining of interphase chromosome breaks in low passage normal human fibroblasts," Radiat. Res.111, 385-405 (1987).

30.M. Carlone, "Determining the $\alpha / \beta$ ratio for prostate cancer using clinically measured dose response data," Thesis for the degree of Doctor in Philosophy. Dept. of Physics. Carleton University. (2003).

31. A.T. Natarajan et al., "Molecular mechanisms involved in the production of chromosomal aberrations," Mutat. Res. 69, 293-305 (1980).

32.P.E. Bryant, "Enzymatic restriction of mammalian cell DNA using Pvull and BamH1: evidence for the double strand break origin of chromosomal aberations", Int. J. Radiat. Biol. 46, 52-65 (1984).

33.E. Pastwa et al. "Repair of Radiation-induced DNA double-strand breaks is dependent upon radiation quality and the structural complexity of double-strand breaks," Radiat. Res. 159, 251-261 (2003).

34.D. Ristic, M. Modesti, R. Kanaar, C. Wyman, "Rad52 and Ku bind to different DNA structures produced early in double-strand break repair," Nucleic Acids Res. 31, 5229-37 (2003).

35.M. Zaider, "There is no mechanistic basis for the use of the linearquadratic expression in cellular survival analysis," Med. Phys. 25, 791-2 (1998).

36.D.E. Lea, Actions of Radiations on Living Cells, Cambridge University Press, London (1946).

37.C.A. Tobias, "The repair-misrepair model in radiobiology: comparison to other models," Radiat. Res. Suppl. 8, S77-S95 (1985).

38.J. Kiefer, Quantitative Mathematical Models in Radiation Biology, Springer, New York (1988). 
39.W. Sontag, "Comparison of six different models describing survival of mammalian cells after irradiation," Radiat. Environ. Biophysics 29, 185201 (1990).

40. B. Fertil, I. Reydellet and P.J. Deschavanne, "A benchmark of cell survival models using survival curves for human cells after completion of repair of potentially lethal damage," Radiat. Res. 138, 61-69 (1994).

41. K.H. Chadwick and H.P.Leenhouts, The molecular theory of radiation biology, Springer-Verlag, (1981).

42. R.G. Dale, "The application of the linear-quadratic dose-effect equation to fractionated and protracted radiotherapy," Br. J. Radiol.58, 515-528 (1985).

43. M.C. Carlone, D.E. Wilkins, B. Nyiri and G.P. Raaphorst, "Clinical radiobiology, early and late responding tissues, and hypofractionation for prostate cancer," Canadian Organization of Medical Physicists. InterACTIONS, 49, 14-20 (2003).

44.D.J. Brenner and E.G. Hall, "Fractionation and protraction for radiotherapy of prostate carcinoma," Int. J. Radiat. Oncol. Biol. Phys. 43, 1095-1101 (1999).

45. M. Carlone, D. Wilkins and P. Raaphorst, "The modified linear-quadratic model of Guerrero and $\mathrm{Li}$ can be derived from mechanistic basis and exhibits linear-quadratic-linear behavior," Phys. Med. Biol. 50, L9-L15 (2005).

46. B. Marples, P. Lambin, K. A. Skov and M. Joiner, "Low dose hyperradiosensitivity and increased radioresistance in mammalian cells," Int. J. Radiat. Biol. 71, No. 6, 721-735 (1997).

47. A. Chalmers, P. Johnston, M. Woodcock, M. Joiner and B. Marples, "PARP-1, PARP-2, and the cellular response to low doses of ionizing radiation," Int. J. Radiat. Oncol. Biol. Phys. 58, 410-419 (2004). 
48.M.M. Elkind and G.F. Whitmore, The radiobiology of Cultured Mammalian Cells, First Edition, Gordon and Breach, New York-London-Paris (1967).

49.D.P. Heller, "Cell respond uniquely to low-dose ionizing radiation," Lawrence Livermore National Laboratory, S\&TR. August (2003).

50.G.P. Raaphorst and S. Boyden, "Adaptive response and its variation in human normal and tumor cells," Int. J. Radiat. Biol. 75, 865-873 (1999).

51.J. Shadley et al. "Characterization of the adaptive response to ionizing radiation induced by low doses of X-rays to human lymphocytes, " Radiat. Res. 111, 511-517 (1997).

52. M. Joiner et al. "Low-dose hypersensitivity: Current status and possible mechanisms," Int. J. Radiat. Oncol. Biol. Phys. 49, No.2, 379-389 (2001).

53.B. Marples et al. "Low-dose hyper-radiosensitivity: A consequence of ineffective cell cycle arrest of radiation-damaged $\mathrm{G}_{2}$-phase cells," Radiat. Res. 161, 247-255 (2004).

54.D.J. Brenner, A.A. Martinez, G.K. Edmundson, C. Mitchell, H.D. Thames and E.P. Armour," Direct evidence that prostate tumors show high sensitivity to fractionation (low alpha/beta ratio), similar to late-responding normal tissue," Int. J. Radiat. Oncol. Biol. Phys., 52, 6-13, (2002).

55. J. Fowler, R. Chappell, M. Ritter, "Is alpha/beta for prostate tumors really low?," Int. J. Radiat. Oncol. Biol. Phys. 50,1021-1031 (2001).

56.C. King and J. Fowler," A simple analytic derivation suggests that prostate cancer alpha/beta ratio is low," Int. J. Radiat. Oncol. Biol. Phys. 51, 213214 (2001).

57.J. Wang et al., "How low is the alpha/beta ratio for prostate cancer?," Int. J. Radiat. Oncol. Biol. Phys. 55, 194-203 (2003).

58.G. Cowan, Statistical data analysis, Oxford University Press Inc., New York (1998). 
59.S. Brandt, Data analysis. Statistical and computational methods for scientists and engineers, Third Edition, Springer-Verlag New York Inc. (1999).

60.R. Eckhardt, "Stan Ulam, John von Neumann, and the Monte Carlo method," Los Alamos Science, Special Issue 15, 131-137 (1987).

61.W. H. Press, S. A. Teukolsky, W. T. Vetterling, B. P. Flannery, Numerical Recipes in $C$ - The Art of Scientific Computing, Second Edition, Cambridge University Press, Cambridge (1992).

62.C. Malone et al., "Combination interferon- $\alpha 2 a$ and 13-cis-retinoic acid enhances radiosensitization of human malignant glioma cells in vitro," Clinical Cancer Research 5, 417-423 (1999)

63.M Guerrero et al., "Extending the linear-quadratic model for large fraction doses pertinent to stereotactic radiotherapy," Phys. Med. Biol. 494825 4835 (2004).

64.J.C. Lagarias, J. A. Reeds, M. H. Wright, and P. E. Wright, "Convergence Properties of the Nelder-Mead Simplex Method in Low Dimensions," SIAM Journal of Optimization 9, No.1, 112-147 (1998).

65.J.J. Moré and D.C. Sorensen, "Computing a Trust Region Step," SIAM Journal on Scientific and Statistical Computing, 3, 553-572 (1983). 